RESEARCH PAPER RP1320

Part of Journal of Research of the National Bureau of Standards, Volume 25,

August 1940

\title{
PHYSICAL, MINERALOGICAL, AND DURABILITY STUDIES ON THE BUILDING AND MONUMENTAL GRANITES OF THE UNITED STATES
}

\author{
By Daniel W. Kessler, Herbert Insley, and William H. Sligh
}

\begin{abstract}
Tests on 116 samples of granite (including the "black granites") from the principal producing districts of the United States gave range values for various physical properties, as follows: Compressive strength, 7,700 to 53,800 lb/in. ${ }^{2}$; absorption (48-hr immersion), 0.02 to 0.58 percent; porosity, 0.40 to 3.84 percent; weights per cubic foot, 157 to $187 \mathrm{lb}$. The "black granites" (gabbros and basalts) were indicated to be stronger, denser, and less absorptive than the normal granites. Tests on a few selected samples gave a range of values for shearing strength of 3,900 to $4,600 \mathrm{lb} /$ in $^{2}$; modulus of rupture, 1,430 to $5,190 \mathrm{lb} / \mathrm{in} .{ }^{2}$; abrasive hardness, $\left(H_{a}\right) 37$ to 88 ; toughness, 8 to 27 .

In compression, granite is indicated to be stronger than other types of masonry materials except for some quartzites, whereas slate and some of the serpentines are stronger than granite in flexure. The porosity of granite is of the same order as that of the marbles, slates, and quartzites, but much lower than that of most other masonry materials. Although granite ranks as a heavy masonry material, the unit weight of a normal granite is somewhat less than that of marble, slate, and serpentine.

Inspections of granite structures have shown several cases in which the granite scaled to a depth of a few hundredths of an inch over a portion of the surface. Chemical and microscopic studies showed that calcium sulfate was present in the decayed granite but not in newly quarried granite. Experiments were made to determine if calcium sulfate, leached into the granite from external sources or formed in the granite by the action of acids of sulfur on calcite ingredients, would produce scaling. Leaching with a 10-percent solution of sulfuric acid caused some granites to disintegrate within 30 days, but leaching with a saturated solution of calcium sulfate produced no decay in 3 years. It was concluded that the conversion of calcite ingredients to calcium sulfate by acids of sulfur is the probable cause of scaling.
\end{abstract}

\section{CONTENTS}

I. Introduction

II. Samples and test specimens

III. Description of samples_.

IV. Physical tests . _

1. Compressive strength

2. Flexural strength

3. Shearing strength

4. Abrasive hardness

5. Toughness

6. Elasticity

7. Absorption

8. Density

(a) Bulk density

(b) True density 195

(c) Weight per cubic foot

9. Porosity

10. Water transmission rate 
V. Durability studies

1. Observations on structures

2. Studies of granite weathering

3. Studies of weathering agents _.. 200

(a) Frost action

(b) Crystallization tests _................ 201

(c) Temperature and moisture cycle tests

VI. Summary

\section{INTRODUCTION}

This study of granites is a continuation of the program ${ }^{1}$ for determining properties of the building and monumental stones which may be of interest in the selection of materials for specific uses.

One hundred and sixteen samples were collected, and petrographic analyses were made on those granites which had not been previously studied for mineral composition. Tests for compressive strength, porosity, absorption, and density were made on most of the samples to afford comparisons between the various granites. A few selected samples were tested for shearing strength, modulus of rupture, abrasive hardness, toughness, and elasticity, in order to show how these properties for granite compare with those of other masonry materials. Certain durability studies were made to determine what agents are mainly concerned in the weathering of granite.

Since there were only a few samples from 12 States, the results for these are grouped under "Miscellaneous Granites" and those for the 10 main producing States are tabulated separately in geographic order. For convenience in comparing different granites, the results for compressive strength, absorption, density, and porosity have been assembled in one table. Data on other masonry materials, given for comparative purposes in the discussion of different physical properties, were obtained from various sources.

In a study of this kind an important consideration is how well the samples represent the deposits from which they were taken. Each producer was relied upon to select and furnish representative samples of his granite. It should not be assumed, however, that any deposit is uniform throughout. Probably granite from all deposits varies in quality with depth and distance from open joints. The samples used in this study are assumed to be near the average of the products from the quarries represented.

\section{SAMPLES AND TEST SPECIMENS}

The samples were 6 by 6 by 24 in. and were selected by the producers to represent the average product of their quarries. In a few instances two or more samples were submitted from the same quarry to show a range in texture or color. No special finish was required, and most of the samples were split from larger blocks without any further surfacing.

1 D. W. Kessler, Physical and Chemical Tests on the Commercial Marbles of the United States, Tech. Pap. BS 12 (1919) T123.

D. W. Kessler, Permeability of Stone, Tech. Pap. BS 20, 155 (1925) T305.

D. W. Kessler and W. H. Sligh, Physical Properties of the Principal Commercial Limestones Used for Building Construction in the United States, Tech. Pap. BS 21, 497 (1927) T349.

D. W. Kessler and W. H. Sligh, Physical Properties and Weathering Characteristics of Slate, BS J. Research 9, 377 (1932) RP477.

D. W. Kessler, Wear Resistance of Natural Stone Flooring, BS J. Research 11, 635 (1933) RP612.

D. W. Kessler, Action of Hypo Solution on Stone Tanks, J. Research NBS 16, 161 (1936) RP863. 
Others were sawed and a few were polished on one or more faces. The rift faces on some of the samples were not marked by the producers, and for these the rift was judged by inspection or by simple tests.

Five producers supplied prepared specimens for flexure tests. Other specimens for the different tests were prepared at the National Bureau of Standards. For most tests these consisted of cores approximately 2.1 in. in diameter and $2.5 \mathrm{in}$. high. These were cut with core drills using No. 60 silicon carbide and water. The cores were cut to a depth of 3 in., broken out, and finished on the ends by grinding. Seven cores from each sample with the axes parallel to the rift and 7 with the axes perpendicular to the rift were prepared, as indicated in figure 1.

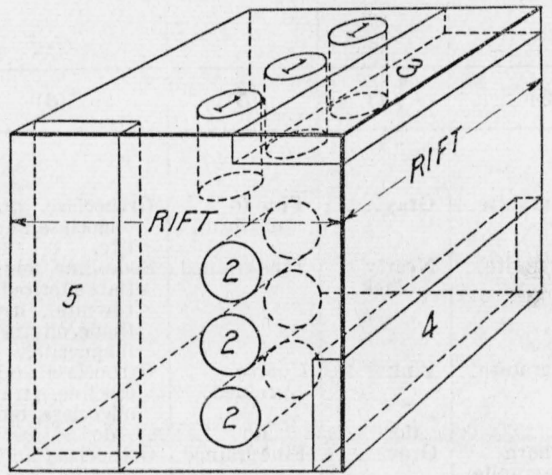

FIGURE 1.-Diagram showing manner of preparing specimens with respect to rift direction.

Cores marked "1" are cut perpendicular to rift and cores marked "2" are parallel to rift. Flexural test specimens 3 and 4 are for test perpendicular to rift and 5 parallel.

The ends of specimens used in the compression tests were finished with special care.

The specimens for flexure tests were 12 by 4 by $1 \mathrm{in}$., and, in these tests, they were broken in two equal parts. These parts served for shearing tests without further modification. The 2- by 2- by 1 -in. specimens used for abrasive hardness tests were, in most cases, prepared from the ends of flexure test specimens, but in some cases they were sawed out of larger blocks with silicon-carbide wheels. The specimens for toughness tests were $2.5 \mathrm{~cm}$ (approximately $1 \mathrm{in}$.) in diameter by $2.5 \mathrm{~cm}$ high.

\section{DESCRIPTION OF SAMPLES}

The granites described in table 1 are assigned serial numbers, which are used to identify them in succeeding tables. The mineral constituents and mineral alterations are based on microscopic studies made at this Bureau or at other laboratories, as shown in column 12. 
TABLE 1.-Description of

[Calcium carbonate in column 9 was determined from that part of the sample which was soluble in 1 part of $b=$ porosity, $c=$ absorption, $d=$ water transmission rate, $e=$ deterioration in the soaking and drying test. "-" 10 percent or more below average. When no sign follows a letter, the results were within 10 percent

\begin{tabular}{|c|c|c|c|c|c|c|}
\hline & \multirow[b]{2}{*}{ Source } & \multirow[b]{2}{*}{ Classification } & \multirow[b]{2}{*}{ Color } & \multirow[b]{2}{*}{ Texture } & \multicolumn{2}{|c|}{$\begin{array}{l}\text { Mineral constituents in descending } \\
\text { order of abundance }\end{array}$} \\
\hline $\begin{array}{l}\dot{0} \\
\text { Z } \\
\text { 㑒 } \\
\text { कू }\end{array}$ & & & & & Major & Minor \\
\hline (1) & (2) & (3) & (4) & (5) & (6) & $(\boldsymbol{7})$ \\
\hline 1 & $\begin{array}{l}\text { Vinalhaven, } \\
\text { Maine. }\end{array}$ & Biotite granite.. & Gray ... & $\begin{array}{l}\text { Fine to } \\
\text { medium. }\end{array}$ & $\begin{array}{l}\text { Orthoclase, quartz, } \\
\text { oligoclase, and bio- } \\
\text { tite. }\end{array}$ & \\
\hline 2 & .................. & Biotite granite.. & $\begin{array}{l}\text { Nearly } \\
\text { black. }\end{array}$ & $\begin{array}{l}\text { Coarse } \\
\text { grained. }\end{array}$ & $\begin{array}{l}\text { Soda-lime feldspars } \\
\text { (labradorite to by- } \\
\text { townite), hypers- } \\
\text { thene, olivine, and } \\
\text { magnetite. } \\
\text { Orthoclase and mi- } \\
\text { crocline, quart } z \text {, } \\
\text { oligoclase, biotite. }\end{array}$ & $\begin{array}{l}\text { Magnetite, zir- } \\
\text { con, and apa- } \\
\text { tite. }\end{array}$ \\
\hline 5 & do & $\begin{array}{l}\text { Biotite horn- } \\
\text { blende granite. }\end{array}$ & Gray......... & Fine grained. & $\begin{array}{l}\text { Orthoclase and mi- } \\
\text { crocline, smoky } \\
\text { quartz, oligoclase, } \\
\text { bi otite, horn- } \\
\text { hlende }\end{array}$ & $\begin{array}{l}\text { Magnetite, ti- } \\
\text { tanite, and } \\
\text { apatite. }\end{array}$ \\
\hline 6 & -.... do & Biotite granite. & $\begin{array}{l}\text { Pinkish } \\
\text { gray. }\end{array}$ & $\begin{array}{l}\text { Coarse } \\
\text { grained. }\end{array}$ & $\begin{array}{l}\text { Orthoclase and mi- } \\
\text { crocline, quartz, } \\
\text { oligoclase, and bio- } \\
\text { tite. }\end{array}$ & $\begin{array}{l}\text { Magnetite, zir- } \\
\text { con, and apa- } \\
\text { tite. }\end{array}$ \\
\hline 7 & $\begin{array}{l}\text { Mt. Desert, } \\
\text { Maine. }\end{array}$ & ................ & $\begin{array}{l}\text { Gray } \\
\text { with } \\
\text { tinge of } \\
\text { pink. }\end{array}$ & $\begin{array}{l}\text { Medium } \\
\text { grained. }\end{array}$ & $\begin{array}{l}\text { Orthoclase, plagio- } \\
\text { c las e, s m ok y } \\
\text { quartt, oligoclase, } \\
\text { and biotite. }\end{array}$ & $\begin{array}{l}\text { Apatite and cal- } \\
\text { cite. }\end{array}$ \\
\hline 8 & .................. & ..... do & Gray & Fine grained. & $\begin{array}{l}\text { Orthoclase, smoky } \\
\text { quartz, oligoclase, } \\
\text { and biotite. }\end{array}$ & Magnetite. \\
\hline 9 & $\begin{array}{l}\text { Jonesboro, } \\
\text { Maine. }\end{array}$ & -.............. & $\begin{array}{l}\text { Pinkish } \\
\text { gray. }\end{array}$ & $\begin{array}{l}\text { Medium to } \\
\text { coarse. }\end{array}$ & $\begin{array}{l}\text { Orthoclase, smoky } \\
\text { quart, oligoclase, } \\
\text { and biotite. }\end{array}$ & $\begin{array}{l}\text { Magnetite, } \\
\text { muscovite, } \\
\text { zoisite, and } \\
\text { chlorite. }\end{array}$ \\
\hline 10 & $\begin{array}{l}\text { Long Cove, } \\
\text { Maine. }\end{array}$ & $\begin{array}{l}\text { Biotite- } \\
\text { muscovite } \\
\text { granite. }\end{array}$ & $\begin{array}{r}\text { Bluish } \\
\text { gray. }\end{array}$ & $\begin{array}{l}\text { Fine to } \\
\text { medium. }\end{array}$ & $\begin{array}{l}\text { Microcline and or- } \\
\text { thoclase, quartz, } \\
\text { oligoclase, biotite, } \\
\text { and muscovite. }\end{array}$ & $\begin{array}{l}\text { Garnet, zircon, } \\
\text { apatite, and } \\
\text { chlorite. }\end{array}$ \\
\hline 11 & $\begin{array}{l}\text { North Jay, } \\
\text { Maine. }\end{array}$ & $\begin{array}{l}\text { Biotite- } \\
\text { muscovite } \\
\text { granite. }\end{array}$ & $\begin{array}{l}\text { Light } \\
\text { gray. }\end{array}$ & Finegrained. & do & $\begin{array}{l}\text { Garnet, magne- } \\
\text { tite, and apa- } \\
\text { tite. }\end{array}$ \\
\hline 12 & $\begin{array}{l}\text { Stonington, } \\
\text { Maine. }\end{array}$ & Biotite granite.. & $\begin{array}{l}\text { Gray with } \\
\text { lavender } \\
\text { tint. }\end{array}$ & $\begin{array}{l}\text { Coarse } \\
\text { grained. }\end{array}$ & $\begin{array}{l}\text { Orthoclase and mi- } \\
\text { crocline, smoky } \\
\text { quartz, oligoclase, } \\
\text { and biotite. }\end{array}$ & $\begin{array}{l}\text { M uscovit e, } \\
\text { magnetite, ti- } \\
\text { tanite, zircon, } \\
\text { and pyrite. }\end{array}$ \\
\hline 13 & $\begin{array}{l}\text { Frankfort, } \\
\text { Maine. }\end{array}$ & Biotite granite.. & Gray....... & Medium... & ... do do & $\begin{array}{c}\text { Magnetite and } \\
\text { titanite. }\end{array}$ \\
\hline 14 & $\begin{array}{l}\text { Lincolnville, } \\
\text { Maine. }\end{array}$ & $\begin{array}{l}\text { Muscovite- } \\
\text { biotite } \\
\text { granite. }\end{array}$ & $\begin{array}{l}\text { Bluish } \\
\text { gray. }\end{array}$ & Fine grained. & $\begin{array}{l}\text { Orthoclase and mi- } \\
\text { crocline, smoky } \\
\text { quartz, oligoclase, } \\
\text { muscovite, and bi- } \\
\text { otite. }\end{array}$ & A patite......... \\
\hline 15 & $\begin{array}{l}\text { St. George, } \\
\text { Maine. }\end{array}$ & $\begin{array}{l}\text { Biotite- } \\
\text { muscovite } \\
\text { granite. }\end{array}$ & ...do do........ & $\begin{array}{l}\text { Fine to } \\
\text { medium. }\end{array}$ & $\begin{array}{l}\text { Microcline and or- } \\
\text { thoclase, quartz, } \\
\text { oligoclase, biotite, } \\
\text { and muscovite. }\end{array}$ & $\begin{array}{l}\text { Garnet, zircon, } \\
\text { apatite, and } \\
\text { chlorite. }\end{array}$ \\
\hline
\end{tabular}




\section{granites and their uses}

$\mathrm{HCl}$ and 9 parts of water. Physical properties in column 10 are denoted as follows: $a=$ compressive strength, The signs "+" following letters denote that the results of tests were 10 percent or more above average and of average. Test values for the various samples are given in table 3 and figure 8.]

\begin{tabular}{|c|c|c|c|c|c|}
\hline Mineral alterations & 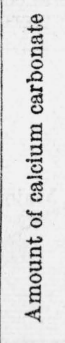 & $\begin{array}{l}\text { Physical char- } \\
\text { acteristics }\end{array}$ & Main uses & 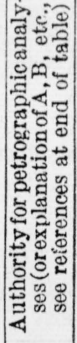 & $\begin{array}{l}\text { Remarks and year } \\
\text { production started }\end{array}$ \\
\hline (8) & (9) & (10) & (11) & $(\mathbf{1} 2)$ & (13) \\
\hline $\begin{array}{l}\text { Oligoclase partly altered } \\
\text { to kaolin and white } \\
\text { mica. }\end{array}$ & $\begin{array}{l}\text { Per- } \\
\text { ient } \\
0.56 \\
3.20\end{array}$ & $\begin{array}{l}a+, b, c, d-, e- \\
a+, b-, c-, d-\end{array}$ & $\begin{array}{l}\text { Paving blocks.- } \\
\text { Monumental }\end{array}$ & A... & $\begin{array}{l}\text { Some porphyritic feld- } \\
\text { spars. } \\
\text { Material takes a high } \\
\text { polish. }\end{array}$ \\
\hline $\begin{array}{l}\text { Oligoclase partly altered } \\
\text { to kaolin and white } \\
\text { mica. }\end{array}$ & 0.31 & $\underset{e-}{a-}, b+, c, d+$, & $\begin{array}{l}\text { Constructional and } \\
\text { architectural } 1\end{array}$ & A... & $\begin{array}{l}\text { Considerable reduction } \\
\text { in strength for wet } \\
\text { specimens. }\end{array}$ \\
\hline $\begin{array}{l}\text { Orthoclase slightly al- } \\
\text { tered to kaolin and } \\
\text { white mica. }\end{array}$ & $\begin{array}{l}.49 \\
.38\end{array}$ & $\begin{array}{l}a, b-, c-, a- \\
a+, b-, c-, d-\end{array}$ & Paving blocks........ & A... & $\begin{array}{l}\text { Porphyritic feldspars } \\
\text { up to } 1 / 4 \text { in. (1887.) }\end{array}$ \\
\hline $\begin{array}{l}\text { Some secondary calcite } \\
\text { in the oligoclase. }\end{array}$ & .25 & $\begin{array}{l}a+, b+, e-, d-, \\
e- \\
\begin{array}{c}a+, b-, c, d- \\
e-\end{array}\end{array}$ & $\begin{array}{l}\begin{array}{c}\text { Architectural and } \\
\text { constructional }^{3}\end{array} \\
\text { Paving blocks........ }\end{array}$ & A... & $\begin{array}{l}\text { Sample showed large } \\
\text { reduction in strength } \\
\text { for wet condition. } \\
\text { (1880). } \\
\text { Sample has porphyritic } \\
\text { feldspars over } 1 / 4 \text { in. } \\
\text { in length. }\end{array}$ \\
\hline $\begin{array}{l}\text { Oligoclase considerably } \\
\text { altered to kaolin and } \\
\text { white mica; biotite to } \\
\text { chlorite. }\end{array}$ & .79 & $a, b+, c, d-, e+$ & $\underset{\text { constructional }}{\text { Arrechitectural }}$ & A... & $\begin{array}{l}\text { in length. } \\
\text { Smoky quartz areas } \\
\text { are prominent. }\end{array}$ \\
\hline & 0.36 & $a, b, c+, d-, e+$ & $\underset{\text { monumental } 6}{\text { Architectural }}$ and & A... & $\begin{array}{l}\text { The sample showed a } \\
\text { considerable reduc- } \\
\text { tion in strength for } \\
\text { the wet condition. } \\
\text { (1873.) } \\
\text { Strength values were } \\
\text { rather variable. }\end{array}$ \\
\hline $\begin{array}{l}\text { Oligoclase much altered } \\
\text { to kaolin and white } \\
\text { mica. }\end{array}$ & .49 & $a-, b, c, d-\ldots$ & Architectural $7 . . .$. & A... & $\begin{array}{l}(1872 .) \\
(1872 .)\end{array}$ \\
\hline $\begin{array}{l}\text { Secondary chlorite kaolin } \\
\text { and white mica. }\end{array}$ & .32 & $\begin{array}{l}\underset{e-}{a+, b}, c+, d+, \\
a-, c+\ldots \ldots .\end{array}$ & Monumental & A... & $\begin{array}{l}\text { The large water trans- } \\
\text { mission value indi- } \\
\text { cates open pore struc- } \\
\text { ture. (1851.) } \\
\text { (1875.) }\end{array}$ \\
\hline $\begin{array}{l}\text { Oligoclase partly altered } \\
\text { to white mica. }\end{array}$ & .25 & $a-\ldots \ldots$ & $\underset{\text { monumental. }}{\text { Architectural }}$ and & A... & (1870.) \\
\hline
\end{tabular}


TABLE 1.-Description of granites

[Calcium carbonate in column 9 was determined from that part of the sample which was soluble in 1 part of $b=$ porosity, $c=$ absorption, $d=$ water transmission rate, $e=$ deterioration in the soaking and drying test. "-" 10 percent or more below average. When no sign follows a letter, the results were within 10 percent

\begin{tabular}{|c|c|c|c|c|c|c|}
\hline \multirow[b]{2}{*}{ 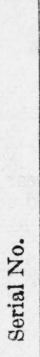 } & \multirow[b]{2}{*}{ Source } & \multirow[b]{2}{*}{ Classification } & \multirow[b]{2}{*}{ Color } & \multirow[b]{2}{*}{ Texture } & \multicolumn{2}{|c|}{$\begin{array}{l}\text { Mineral constituents in descending } \\
\text { order of abundance }\end{array}$} \\
\hline & & & & & Major & Minor \\
\hline (1) & (2) & (3) & (4) & $(5)$ & (6) & $(\boldsymbol{y})$ \\
\hline 16 & $\begin{array}{l}\text { Highpine, } \\
\text { Maine. }\end{array}$ & Biotite granite.. & Pink ...... & Coarse $\ldots . . .$. & $\begin{array}{l}\text { Microcline and or- } \\
\text { thoclase, smoky } \\
\text { quartz, oligoclase, } \\
\text { and biotite. }\end{array}$ & $\begin{array}{l}\text { Magnetite, zir- } \\
\text { con, musco- } \\
\text { vite, and chlo- } \\
\text { rite. }\end{array}$ \\
\hline 17 & $\begin{array}{l}\text { Concord, N. } \\
\text { H. }\end{array}$ & $\begin{array}{l}\text { Muscovite - bio- } \\
\text { tite granite. }\end{array}$ & $\begin{array}{l}\text { Bluish } \\
\text { gray. }\end{array}$ & $\begin{array}{l}\text { Fine to me- } \\
\text { dium. }\end{array}$ & $\begin{array}{l}\text { Microcline and or- } \\
\text { thoclase, quartz, } \\
\text { oligoclase - albite, } \\
\text { muscovite, and } \\
\text { biotite. }\end{array}$ & $\begin{array}{l}\text { rite. } \\
\text { Magnetite, apa- } \\
\text { tite, zircon, } \\
\text { and rutile. }\end{array}$ \\
\hline 18 & $\begin{array}{l}\text { Redstone, } \\
\text { N. H. }\end{array}$ & Biotite granite.. & Pink...... & $\begin{array}{l}\text { Coarse } \\
\text { grained. }\end{array}$ & $\begin{array}{l}\text { Orthoclase, smoky } \\
\text { quartz, (oligo- } \\
\text { clase-albite), and } \\
\text { biotite. }\end{array}$ & $\begin{array}{l}\text { Magnetite, py- } \\
\text { rite, apatite, } \\
\text { fluorite, and } \\
\text { zircon. }\end{array}$ \\
\hline 19 & ..... do ... & $\begin{array}{l}\text { Biotite-horn- } \\
\text { blende granite. }\end{array}$ & $\begin{array}{l}\text { Dark } \\
\text { green. }\end{array}$ & Coarse...... & $\begin{array}{l}\text { Orthoclase, smoky } \\
\text { quartz, plagioclase } \\
\text { (albite-oligoclase), } \\
\text { biotite, and horn- } \\
\text { blende. }\end{array}$ & $\begin{array}{l}\text { Magnetite, fluo- } \\
\text { rite, allanite, } \\
\text { and zircon. }\end{array}$ \\
\hline 20 & Mason, N. H. & $\begin{array}{l}\text { Quartz-biotite- } \\
\text { monzonite. }\end{array}$ & Gray...... & $\begin{array}{l}\text { Medium } \\
\text { grained. }\end{array}$ & $\begin{array}{l}\text { Pot a sh feldspar, } \\
\text { quartz, plagioclase, } \\
\text { and biotite. }\end{array}$ & $\begin{array}{l}\text { Muscovite, mag- } \\
\text { netite, and } \\
\text { epidote. }\end{array}$ \\
\hline 21 & Milford, N.H. & $\begin{array}{l}\text { Quartz-monzon- } \\
\text { ite. }\end{array}$ & Light & Finegrained. & $\begin{array}{l}\text { Microcline and or- } \\
\text { thoclase, oligoclase, } \\
\text { smoky quartz, and } \\
\text { biotite. }\end{array}$ & $\begin{array}{l}\text { Allanite, zircon, } \\
\text { and apatite. }\end{array}$ \\
\hline 22 & . . . do . . . . & .... do .... & Dark gray & .... do & $\begin{array}{l}\text { Smoky quartz, oligo- } \\
\text { clase, microcline, } \\
\text { and biotite. }\end{array}$ & $\begin{array}{l}\text { Magnetite, py- } \\
\text { rite, apatite, } \\
\text { allanite, and } \\
\text { zircon. }\end{array}$ \\
\hline 23 & ..... do . ..... & .... do . . . & $\begin{array}{l}\text { Bluish } \\
\text { gray. }\end{array}$ & $\begin{array}{l}\text { Medium } \\
\text { grained. }\end{array}$ & $\begin{array}{l}\text { S mok y quartz, } \\
\text { oligoclase, micro- } \\
\text { cline, and biotite. }\end{array}$ & $\begin{array}{l}\text { zircon. } \\
\text { Magnetite, apa- } \\
\text { tite, pyrite, al- } \\
\text { lanite, and }\end{array}$ \\
\hline 24 & $\begin{array}{l}\text { Fitzwilliams, } \\
\text { N. H. }\end{array}$ & $\begin{array}{l}\text { Biotite-musco- } \\
\text { vite granite. }\end{array}$ & ... do . . . & Fine grained. & $\begin{array}{l}\text { Orthoclase and mi- } \\
\text { crocline, quartz, } \\
\text { oligoclase, biotite, } \\
\text { and muscovite. }\end{array}$ & $\begin{array}{l}\text { zircon. } \\
\text { Rutile }\end{array}$ \\
\hline 25 & Barre, vt. & Biotite granite.. & ... do ...... & .... do . . . . & $\begin{array}{l}\text { Orthoclase, smoky } \\
\text { quartz, (oligoclase- } \\
\text { albite), biotite, } \\
\text { and muscovite. }\end{array}$ & $\begin{array}{l}\text { Titanite, mag- } \\
\text { netite, and py- } \\
\text { rite. }\end{array}$ \\
\hline 26 & ..... do ..... & . . do . . . . & .... do ...... & Medium.... & $\begin{array}{l}\text { Orthoclase and mi- } \\
\text { crocline, smoky } \\
\text { quartz, oligoclase- } \\
\text { albite, biotite, and } \\
\text { muscovite. }\end{array}$ & $\begin{array}{l}\text { Magnetite and } \\
\text { zircon. }\end{array}$ \\
\hline 27 & Derby, Vt... & $\begin{array}{l}\text { Quartz monzon- } \\
\text { ite. }\end{array}$ & ...do........ & $\begin{array}{l}\text { Fine to me- } \\
\text { dium. }\end{array}$ & $\begin{array}{l}\text { Smoky quartz, oligo- } \\
\text { clase, microcline } \\
\text { and orthoclase, } \\
\text { biotite, and mus- } \\
\text { covite. }\end{array}$ & $\begin{array}{l}\text { Apatite, titanite, } \\
\text { allanite, and } \\
\text { rutile. }\end{array}$ \\
\hline 28 & $\begin{array}{l}\text { Woodb ury, } \\
\text { Vt. }\end{array}$ & Biotite granite.. & Light gray. & Coarse ....... & $\begin{array}{l}\text { Microcline and or- } \\
\text { thoclase, quartz, } \\
\text { oligoclase, biotite, } \\
\text { and muscovite. }\end{array}$ & D-mito Sutito \\
\hline 29 & ...... do & ...... do _. & $\begin{array}{l}\text { Bluish } \\
\text { gray. }\end{array}$ & Medium.... & .... do & $\begin{array}{l}\text { Pyrite, apatite, } \\
\text { zircon, and } \\
\text { rutile. }\end{array}$ \\
\hline
\end{tabular}




\section{and their uses-Continued}

$\mathrm{HCl}$ and 9 parts of water. Physical properties in column 10 are denoted as follows: $a=$ compressive strength, The signs "+" following letters denote that the results of tests were 10 percent or more above average and of average. Test values for the various samples are given in table 3 and figure 8.1

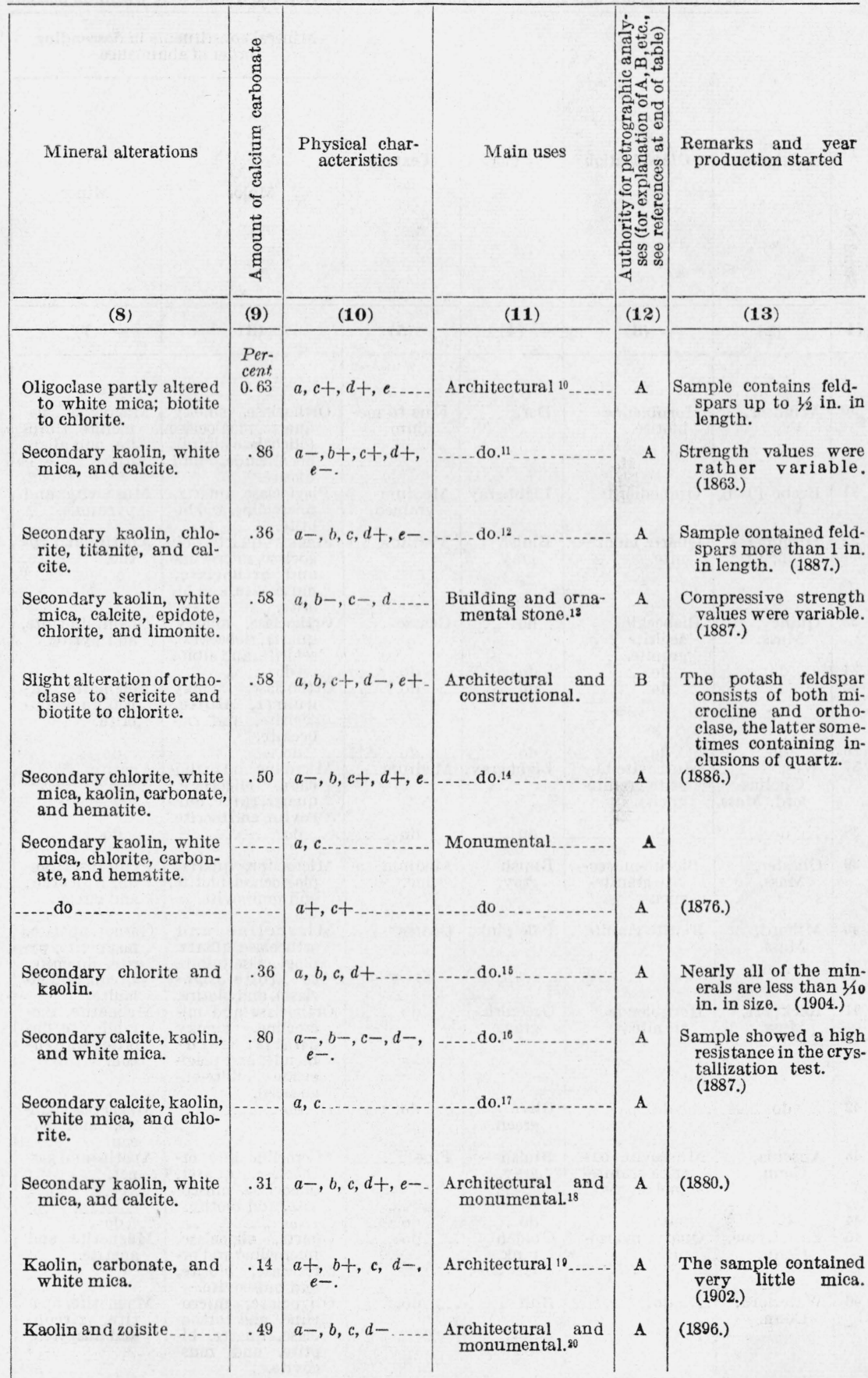


TABLE 1.-Description of granites

[Calcium carbonate in column 9 was determined from that part of the sample which was soluble in 1 part of $b=$ porosity, $c=$ absorption, $d=$ water transmission rate, $e=$ deterioration in the soaking and drying test. "-" 10 percent or more below average. When no sign follows a letter, the results were within 10 percent

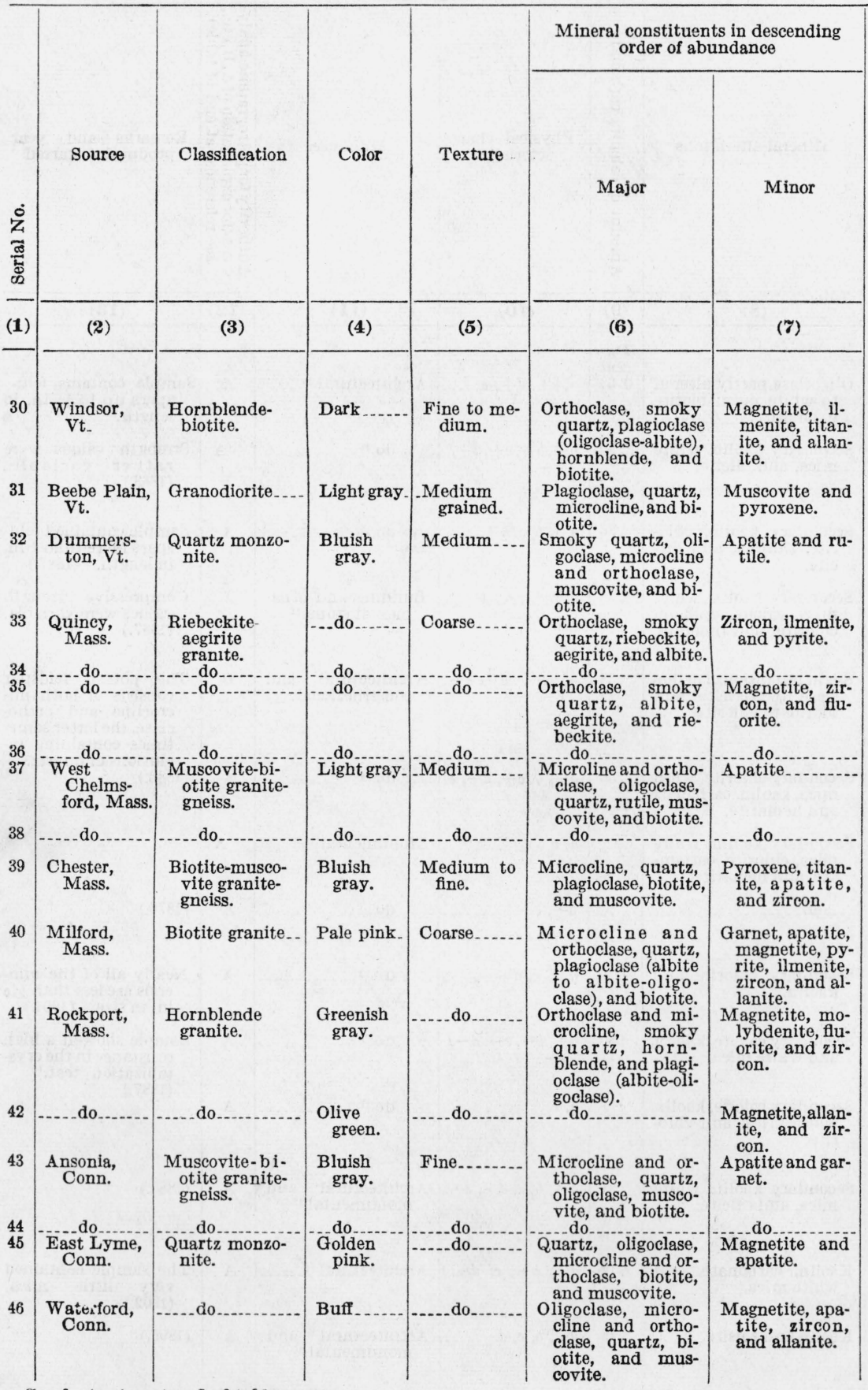

See footnotes at end of table. 


\section{and their uses-Continued}

$\mathrm{HCl}$ and 9 parts of water. Physical properties in column 10 are denoted as follows $a=$ compressive strength The signs "+" following letters denote that the results of tests were 10 percent or more above average and of average. Test values for the various samples are given in table 3 and figure 8.]

\begin{tabular}{|c|c|c|c|c|c|}
\hline Mineral alterations & 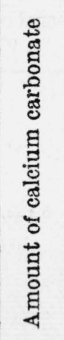 & $\begin{array}{l}\text { Physical char- } \\
\text { acteristics }\end{array}$ & Main uses & 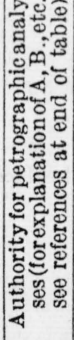 & $\begin{array}{l}\text { Remarks and year } \\
\text { production started }\end{array}$ \\
\hline (8) & (9) & (10 & (11) & (12) & (13) \\
\hline Secondary kaolin & $\begin{array}{l}\text { Per. } \\
\text { cent } \\
0.39\end{array}$ & $a+, b-, c-, d-$ & $\begin{array}{l}\text { Architectural and } \\
\text { monumental. } 21\end{array}$ & A & $\begin{array}{l}\text { Orthoclase is olive } \\
\text { green; the other feld- } \\
\text { spars and hornblende } \\
\text { are darker green. }\end{array}$ \\
\hline $\begin{array}{l}\text { Slight alteration in plagi- } \\
\text { oclase. }\end{array}$ & & $a-, c$ & Monumental & B & \\
\hline $\begin{array}{l}\text { Secondary kaolin, white } \\
\text { mica, epidote, zoisite, } \\
\text { and calcite. }\end{array}$ & & $a, c+\ldots$ & $\begin{array}{l}\text { Architectural and } \\
\text { monumental.2a }\end{array}$ & A & (1877.) \\
\hline $\begin{array}{l}\text { Leucoxene is secondary; } \\
\text { riebeckite partly sec- } \\
\text { ondary. }\end{array}$ & .31 & $a, b+, c, d-, e_{-}$ & Monumental & A & $\begin{array}{l}\text { Strength values are } \\
\text { rather variable. }\end{array}$ \\
\hline $\begin{array}{l}\text { Secondary kaolin, horn- } \\
\text { blende, and chlorite. }\end{array}$ & $\begin{array}{l}.41 \\
.31\end{array}$ & $\begin{array}{l}a, b+, c, d-, e \\
a, b+, c, d+\end{array}$ & do do & $\begin{array}{l}\mathrm{A} \\
\mathrm{A}\end{array}$ & (1846.) \\
\hline $\begin{array}{l}\text { Secondary kaolin, white } \\
\text { mica, carbonate, epi- } \\
\text { dote and chlorite. }\end{array}$ & $\begin{array}{l}.32 \\
.45\end{array}$ & $\begin{array}{l}a, b, c, d- \\
a, b+, c+, d\end{array}$ & $\begin{array}{l}\text { Architectural and } \\
\text { constructional. }{ }^{24}\end{array}$ & $\begin{array}{l}\mathrm{A} \\
\mathrm{A}\end{array}$ & $\begin{array}{l}\text { The sample had a } \\
\text { gneissic structure. } \\
\text { (1881.) }\end{array}$ \\
\hline do & .49 & $a, b+, c+, d+$ & _....do do & $\mathbf{A}$ & Do. \\
\hline $\begin{array}{l}\text { Micas and pyroxene } \\
\text { often corroded. Sec- } \\
\text { andary calcite and } \\
\text { kaolin. }\end{array}$ & 1.17 & $a, b, c, d-\ldots$ & Monumental & B & \\
\hline $\begin{array}{l}\text { Secondary kaolin, white } \\
\text { mica, epidote, zoisite, } \\
\text { leucoxene, chlorite, and } \\
\text { calcite. }\end{array}$ & 0.31 & $a, b, c, d+, e-\ldots$ & $\begin{array}{l}\text { Architectural and } \\
\text { constructional.25 }\end{array}$ & $\mathbf{A}$ & $\begin{array}{l}\text { The sample has an ap. } \\
\text { preciable gneissic } \\
\text { structure. }\end{array}$ \\
\hline $\begin{array}{l}\text { Secondary kaolin and } \\
\text { calcite. }\end{array}$ & (n).... & $c-$ & . do.26 & A & (1855.) \\
\hline $\begin{array}{l}\text { Secondary kaolin, chlo- } \\
\text { rite, and limonite. }\end{array}$ & & $c-\ldots \ldots$ & do. & $\mathbf{A}$ & (1868.) \\
\hline $\begin{array}{l}\text { Secondary kaolin and } \\
\text { calcite. }\end{array}$ & .34 & $\underset{e-c}{a-, b-, c, d-}$ & Constructional.....-. & $\mathbf{A}$ & (1890.) \\
\hline do do . . . & .41 & $a, b, c-, d-\ldots$ & do do & $\mathbf{A}$ & (1890.) \\
\hline $\begin{array}{l}\text { Secondary hematite, ka- } \\
\text { olin, calcite, and white } \\
\text { mica. }\end{array}$ & .58 & $\begin{array}{l}a+, b+, c, d- \\
e+.\end{array}$ & Monumental & A & (1900.) \\
\hline $\begin{array}{l}\text { Secondary white mica, } \\
\text { calcite, chlorite, and } \\
\text { limonite. }\end{array}$ & .56 & $\begin{array}{l}a, b+, c+, d- \\
\text { e+. }\end{array}$ & $\underset{\text { monumental. }{ }^{28}}{\text { Architectural and }}$ & A & $\begin{array}{l}\text { Strength values were } \\
\text { quite variable. } \\
\text { (1878.) }\end{array}$ \\
\hline
\end{tabular}




\section{TABLE 1.-Description of granites}

[Calcium carbonate in column 9 was determined from that part of the sample which was soluble in 1 part of $b=$ porosity, $c=$ absorption, $d=$ water transmission rate, $e=$ deterioration in the soaking and drying test. "-" 10 percent or more below average. When no sign follows a letter, the results were within 10 percent

\begin{tabular}{|c|c|c|c|c|c|c|}
\hline \multirow[b]{2}{*}{ 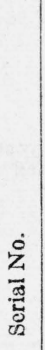 } & \multirow[b]{2}{*}{ Source } & \multirow[b]{2}{*}{ Classification } & \multirow[b]{2}{*}{ Color } & \multirow[b]{2}{*}{ Texture } & \multicolumn{2}{|c|}{$\begin{array}{l}\text { Mineral constituents in descending } \\
\text { order of abundance }\end{array}$} \\
\hline & & & & & Major & Minor. \\
\hline (1) & (2) & (3) & (4) & (5) & (6) & (z) \\
\hline 48 & $\begin{array}{l}\text { Branford, } \\
\text { Conn. } \\
\text { Stony } \\
\text { Creek, } \\
\text { Conn. }\end{array}$ & $\begin{array}{l}\text { Biotite granite } \\
\text { gneiss. }\end{array}$ & $\begin{array}{l}\text { Reddish } \\
\text { gray. } \\
\text { Reddish }\end{array}$ & $\begin{array}{l}\text { Medium to } \\
\text { coarse. } \\
\text { Coarse }\end{array}$ & $\begin{array}{l}\text { Microcline and or- } \\
\text { thoclase, quartz, } \\
\text { oligoclase, biotite, } \\
\text { and muscovite. }\end{array}$ & $\begin{array}{l}\text { Magnetite, py- } \\
\text { rite, apatite, } \\
\text { and zircon. }\end{array}$ \\
\hline 49 & Duke, N. C. & $\begin{array}{l}\text { Quartz-ilmenite } \\
\text { schist }\end{array}$ & Dark gray & Fine grained & $\begin{array}{l}\text { Quartz, ilmenite, } \\
\text { pyroxene, amphi- } \\
\text { bole, and hematite. }\end{array}$ & \\
\hline $\begin{array}{l}50 \\
51\end{array}$ & $\begin{array}{c}\text { Wake For- } \\
\text { est, N. C. }\end{array}$ & Biotite granite. & Bluish & $\begin{array}{l}\text { Fine to } \\
\text { medium. }\end{array}$ & $\begin{array}{l}\text { Orthoclase, micro- } \\
\text { cline, acidic plagi- } \\
\text { oclase, and biotite. }\end{array}$ & $\begin{array}{l}\text { Muscovite, apa- } \\
\text { tite, and zir- } \\
\text { con. }\end{array}$ \\
\hline 53 & $\begin{array}{l}\text { Mt. Airy, } \\
\text { N. C. }\end{array}$ & ..... do & $\begin{array}{c}\text { Light } \\
\text { gray. }\end{array}$ & $\begin{array}{l}\text { Medium } \\
\text { grained. }\end{array}$ & $\begin{array}{l}\text { Oligoclase, ortho- } \\
\text { clase and micro- } \\
\text { cline, quartz, and } \\
\text { and biotite. }\end{array}$ & $\begin{array}{l}\text { Apatite and zir- } \\
\text { con. }\end{array}$ \\
\hline 56 & $\begin{array}{l}\text { Salisbury, } \\
\text { N. C. }\end{array}$ & ...... do & Pink & _... do do...... & $\begin{array}{l}\text { Orthoclase with } \\
\text { small amount of } \\
\text { microcline, plagio- } \\
\text { clase, quartz, and } \\
\text { biotite. }\end{array}$ & $\begin{array}{l}\text { Magnetite and } \\
\text { garnet. }\end{array}$ \\
\hline 57 & ........ do & .... do .. & ... do ... & .... do _.. & .... do & -..._do_... \\
\hline 5 & .... do & ....... do & ... do & ..... do & do do & . \\
\hline 60 & Rion, S. C - & ..... do & $\begin{array}{l}\text { Light } \\
\text { gray. }\end{array}$ & .....do do.... & $\begin{array}{l}\text { Orthoclase and mi- } \\
\text { crocline, plagio- } \\
\text { clase (oligoclase), } \\
\text { quartz, and bio- } \\
\text { tite. }\end{array}$ & $\begin{array}{l}\text { Apatite, zircon, } \\
\text { and iron oxide. }\end{array}$ \\
\hline 61 & $\begin{array}{l}\text { Newberry, } \\
\text { S. C. }\end{array}$ & 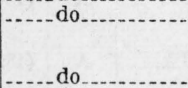 & $\begin{array}{c}\begin{array}{c}\text { Bluish } \\
\text { gray. }\end{array} \\
\text { do }\end{array}$ & $\begin{array}{l}\text { Fine } \\
\text { grained. }\end{array}$ & $\begin{array}{l}\text { Orthoclase with m- } \\
\text { crocline, oligo- } \\
\text { clase, and biotite. }\end{array}$ & $\begin{array}{l}\text { Apatite, zircon, } \\
\text { and magnet- } \\
\text { ite. }\end{array}$ \\
\hline 63 & $\begin{array}{l}\text { Greenwood, } \\
\text { S. C. }\end{array}$ & . & $\begin{array}{l}\text { Light } \\
\text { gray. }\end{array}$ & $\begin{array}{l}\text { Medium } \\
\text { grained. }\end{array}$ & $\begin{array}{l}\text { Microcline and or- } \\
\text { thoclase, oligo- } \\
\text { clase, quartz, and } \\
\text { biotite. }\end{array}$ & $\begin{array}{l}\text { Titanite, zircon, } \\
\text { apatite, and } \\
\text { iron oxide. }\end{array}$ \\
\hline 64 & $\begin{array}{l}\text { Winnsboro, } \\
\text { S. C. }\end{array}$ & ......do.... & ...do....... & ..... do & $\begin{array}{l}\text { Orthoclase and mi- } \\
\text { crocline, plagio- } \\
\text { clase (oligoclase), } \\
\text { quartz, and bio- } \\
\text { tite. }\end{array}$ & $\begin{array}{l}\text { A patite, zircon, } \\
\text { and iron oxide. }\end{array}$ \\
\hline
\end{tabular}

See footnotes at end of table, 


\section{and their uses-Continued}

$\mathrm{HCl}$ and 9 parts of water. Physical properties in column 10 are denoted as follows: $a=$ compressive strength The signs "+" following letters denote that the results of tests were 10 percent or more above average and of average. 'Test values for the various samples are given in table 3 and figure 8.]

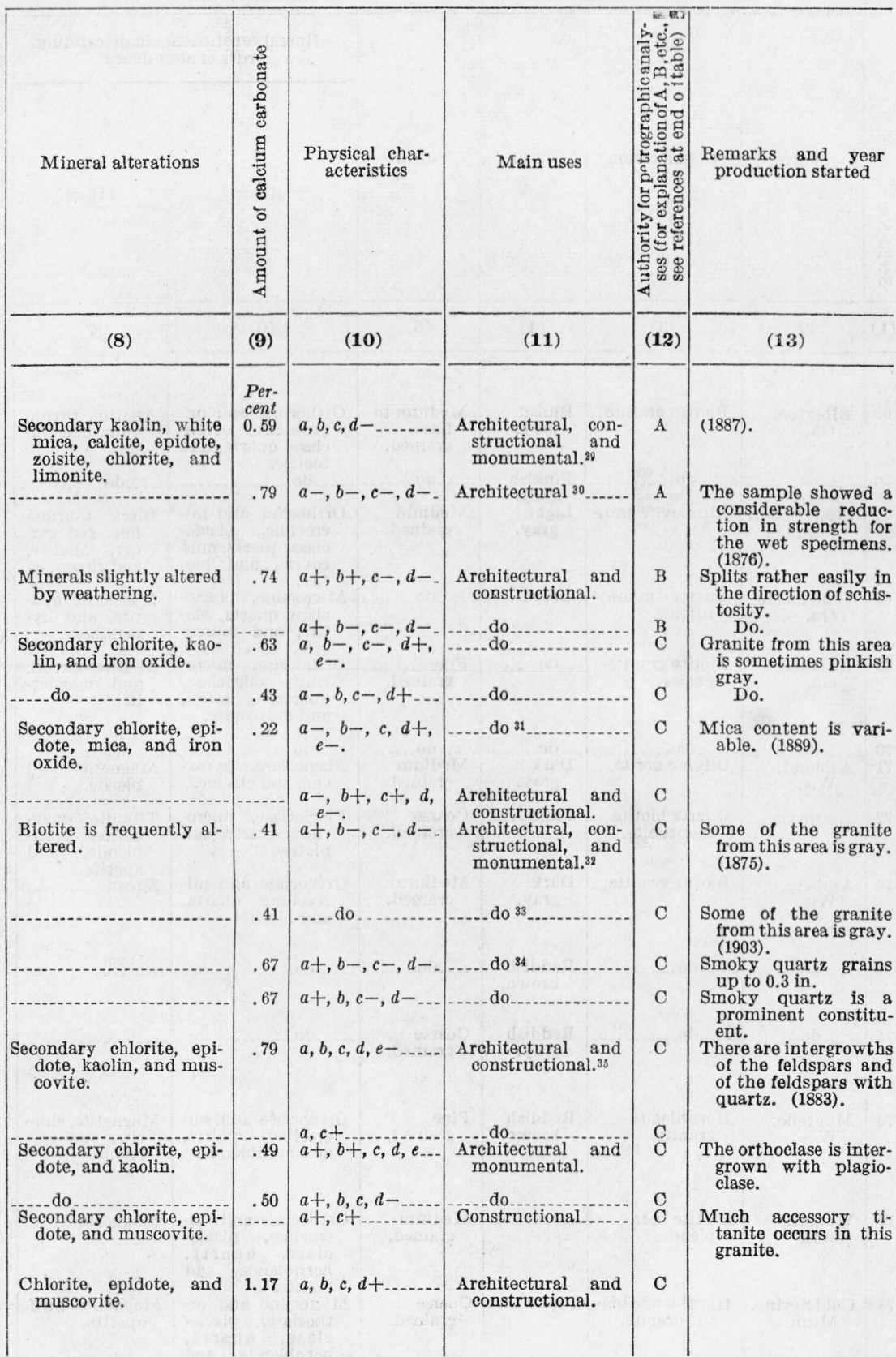


TABLE 1.-Description of granites

[Calcium carbonate in column 9 was determined from that part of the sample which was soluble in 1 part of $b=$ porosity, $c=$ absorption, $d=$ water transmission rate, $e=$ deterioration in the soaking and drying test. "-" 10 percent or more below average. When no sign follows a letter, the results were within 10 percent

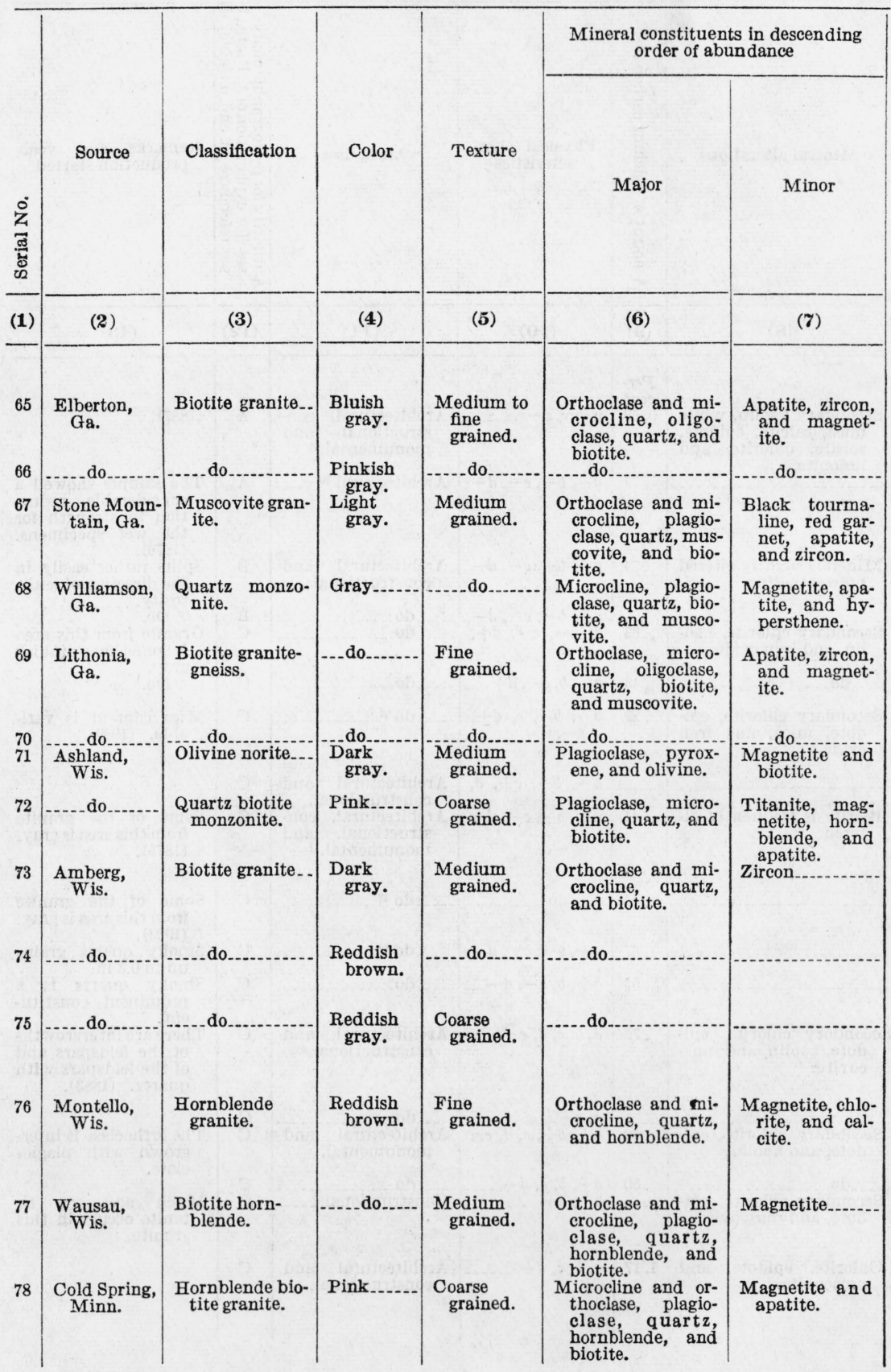

See footnotes at end of table. 


\section{and their uses-Continued}

$\mathrm{HCl}$ and 9 parts of water. Physical properties in column 10 are denoted as follows: $a=$ compressive strength The signs "+" following letters denote that the results of tests were 10 percent or more above average and of average. Test values for the various samples are given in table 3 and figure 8.]

\begin{tabular}{|c|c|c|c|c|c|}
\hline Mineral alterations & 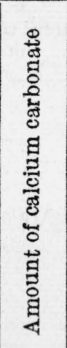 & $\begin{array}{l}\text { Physical char- } \\
\text { acteristics }\end{array}$ & Main uses & 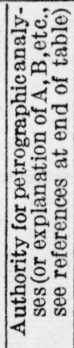 & $\begin{array}{l}\text { Remarks and year } \\
\text { production started }\end{array}$ \\
\hline (8) & $(\mathbf{9})$ & (10) & (11) & (12) & $(13$ \\
\hline $\begin{array}{l}\text { Secondary chlorite and } \\
\text { epidote. }\end{array}$ & $\begin{array}{l}\text { Per- } \\
\text { cent } \\
0.56\end{array}$ & $\begin{array}{l}a+, b+, c+, d- \\
\quad++\end{array}$ & Monumental.......... & $\mathrm{C}$ & $\begin{array}{l}\text { Good contrast between } \\
\text { polished and ham- } \\
\text { mered finishes. }\end{array}$ \\
\hline - & .61 & $a, b, c, d-, e+\ldots$ & . do do & C & \\
\hline Secondary chlorite........ & .31 & $\begin{array}{c}a, b-, c-, d+ \\
e-\end{array}$ & $\begin{array}{l}\text { Architectural and } \\
\text { constructional. }{ }^{36}\end{array}$ & $\mathrm{C}$ & \\
\hline $\begin{array}{l}\text { Plagioclase, sometimes } \\
\text { altered to calcite and } \\
\text { sericite. }\end{array}$ & 1. 42 & $a-, b, c, d-, e+$ & Constructional...... & B & $\begin{array}{l}\text { Occasional inter- } \\
\text { growths between } \\
\text { quartz and feldspars. }\end{array}$ \\
\hline $\begin{array}{l}\text { Secondary chlorite, epi- } \\
\text { dote, kaolin, and white } \\
\text { mica. }\end{array}$ & -n..... & $a, b-, c-\ldots .$. & $\begin{array}{l}\text { Curbing, paving, } \\
\text { and crushed stone. }\end{array}$ & C & $\begin{array}{l}\text { The banded structure } \\
\text { is caused by a partial } \\
\text { segregation of the } \\
\text { biotite, quartz, and } \\
\text { feldspars into layers. } \\
\text { Do. }\end{array}$ \\
\hline $\begin{array}{l}\text { Slight alteration of pla- } \\
\text { gioclase to calcite or } \\
\text { sericite. }\end{array}$ & 1.76 & $a-, b+, c-, d-$ & $\begin{array}{l}\text { Architectural and } \\
\text { monumental. }\end{array}$ & B & \\
\hline $\begin{array}{l}\text { Plagioclase frequently } \\
\text { weathered to calcite } \\
\text { and sericite at center } \\
\text { of crystals. }\end{array}$ & 1. 24 & $\begin{array}{l}a-, b+, c+, d+, \\
e-\end{array}$ & $\begin{array}{l}\text { Architectural and } \\
\text { constructional. }\end{array}$ & B & \\
\hline $\begin{array}{l}\text { Evidence of alteration in } \\
\text { some of the feldspars. }\end{array}$ & .67 & 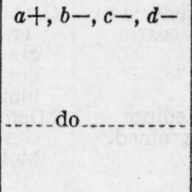 & $\begin{array}{l}\text { Architectural and } \\
\text { monumental. }\end{array}$ & $\mathrm{D}$ & $\begin{array}{l}\text { Surface granulation of } \\
\text { the components and } \\
\text { bending of the longer } \\
\text { feldspars are in evi- } \\
\text { dence. (1896.) } \\
\text { Mica shows some de- } \\
\text { gree of alignment and } \\
\text { segregation into } \\
\text { sheets. }\end{array}$ \\
\hline $\begin{array}{l}\text { Some feldspars partly } \\
\text { altered to epidote. }\end{array}$ & .32 & $a-, b-, c-, d-$ & ...... do............. & D & $\begin{array}{l}\text { A striking combina- } \\
\text { tion of colors. } \\
\text { Quartz and feldspars } \\
\text { show surface granu- } \\
\text { lation. }\end{array}$ \\
\hline $\begin{array}{l}\text { Magnetite partly altered } \\
\text { to hematite. }\end{array}$ & 0.38 & $\begin{array}{l}a+, b-, c-, d- \\
e+.\end{array}$ & Monumental ${ }^{37} \ldots$ & D......... & $\begin{array}{l}\text { The color is caused by } \\
\text { hematite, which oc- } \\
\text { curs mainly along } \\
\text { the cleavage planes } \\
\text { of the feldspars. } \\
\text { (1880.) } \\
\text { Both quartz and feld- } \\
\text { spar show surface } \\
\text { granulation. (1895.) }\end{array}$ \\
\hline & .68 & $a-, b+, c-, d-$ & $\begin{array}{l}\text { Architectural and } \\
\text { monumental. }{ }^{88}\end{array}$ & E \& F & $-(1913)$. \\
\hline
\end{tabular}


TABLE 1.-Description of granites

[Calcium carbonate in column 9 was determined from that part of the sample which was soluble in 1 part of $b=$ porosity, $c=$ absorption, $d=$ water transmission rate, $e=$ deterioration in the soaking and drying test. "_" 10 percent or more below average. When no sign follows a letter, the results were within 10 percent

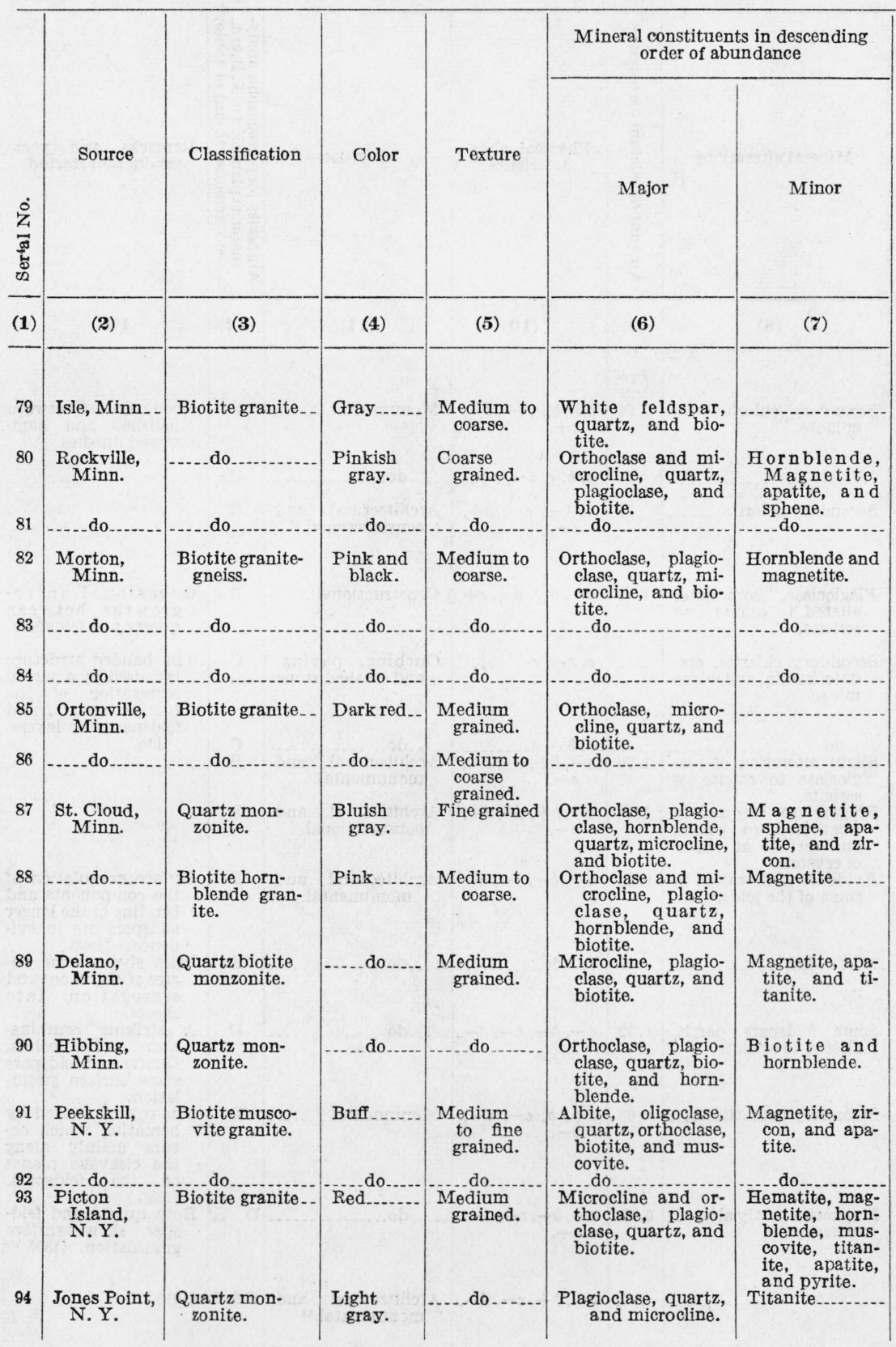

See footnotes at end of table. 
and their uses-Continued

$\mathrm{HCl}$ and 9 parts of water. Physical properties in column 10 are denoted as follows: $a=$ eompressive strength, The signs "+" following letters denote that the results of tests were 10 percent or more above average and of average. Test values for the various samples are given in table 3 and figure 8.]

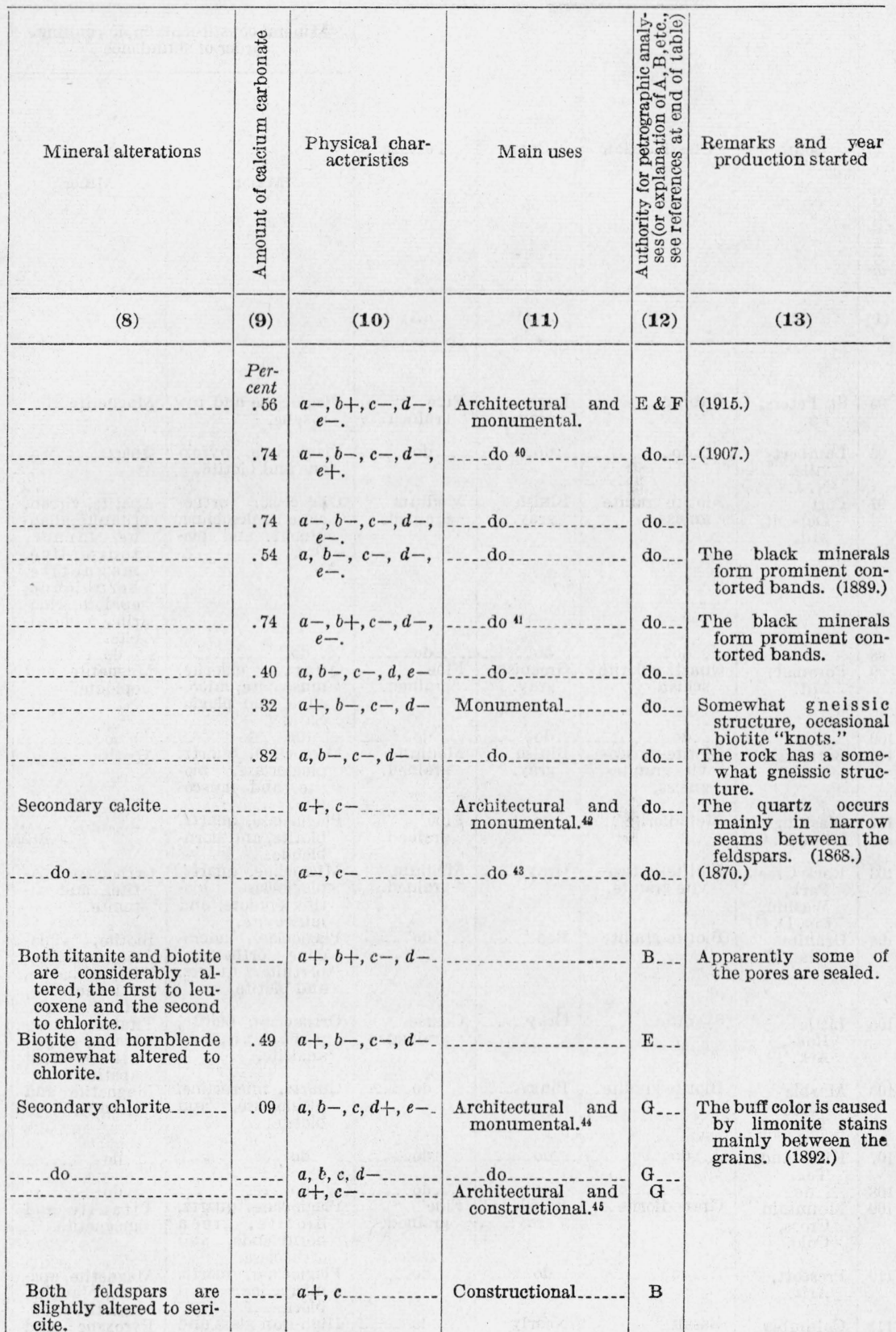


TABLE 1.-Description of granites

[Calcium carbonate in column 9 was determined from that part of the sample which was soluble in 1 part of $b=$ porosity, $c=$ absorption, $d=$ water transmission rate, $e=$ deterioration in the soaking and drying test. "- 10 percent or more below average. When no sign follows a letter, the results were within 10 percent

\begin{tabular}{|c|c|c|c|c|c|c|}
\hline & \multirow[b]{2}{*}{ Source } & \multirow[b]{2}{*}{ Classification } & \multirow[b]{2}{*}{ Color } & \multirow[b]{2}{*}{ Texture } & \multicolumn{2}{|c|}{$\begin{array}{l}\text { Mineral constituents in descending } \\
\text { order of abundance }\end{array}$} \\
\hline 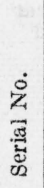 & & & & & Major & Minor \\
\hline (1) & (2) & (3) & (4) & (5) & (6) & $(\boldsymbol{\gamma})$ \\
\hline 95 & $\begin{array}{l}\text { St. Peters, } \\
\text { Pa. }\end{array}$ & Gabbro ............ & $\begin{array}{l}\text { Dark } \\
\text { gray. }\end{array}$ & $\begin{array}{l}\text { Fine } \\
\text { grained. }\end{array}$ & $\begin{array}{l}\text { Plagioclase and py- } \\
\text { roxene. }\end{array}$ & Magnetite...... \\
\hline 96 & $\begin{array}{l}\text { Lambert- } \\
\text { ville, } \\
\text { N.J. }\end{array}$ & .... do... & -. do & . do & $\begin{array}{l}\text { Plagioclase, pyrox- } \\
\text { ene and biotite. }\end{array}$ & Quartz \\
\hline 97 & $\begin{array}{l}\text { Port } \\
\text { Deposit, } \\
\text { Md. }\end{array}$ & $\begin{array}{l}\text { Biotite granite } \\
\text { gneiss. }\end{array}$ & $\underset{\text { gray. }}{\text { Bluish }}$ & $\begin{array}{l}\text { Medium } \\
\text { grained. }\end{array}$ & $\begin{array}{l}\text { Oligoclase, ortho- } \\
\text { clase microcline, } \\
\text { quartz, and bio- } \\
\text { tite. }\end{array}$ & $\begin{array}{l}\text { Apatite, zircon, } \\
\text { titanite, allan- } \\
\text { ite, garnet, } \\
\text { tourmaline, } \\
\text { magnetite, } \\
\text { hornblende, } \\
\text { epidote, chlo- } \\
\text { rite, and cal- } \\
\text { cite. }\end{array}$ \\
\hline 99 & $\begin{array}{l}\text { Potomac, } \\
\text { Md. }\end{array}$ & $\begin{array}{l}\text { Quartz chlorite } \\
\text { schist. }\end{array}$ & $\begin{array}{l}\text { Greenish } \\
\text { gray. }\end{array}$ & $\begin{array}{l}\text { Fine } \\
\text { grained. }\end{array}$ & $\begin{array}{l}\text { Quartz, chlorite, } \\
\text { muscovite, micro- } \\
\text { cline, and plagio- } \\
\text { clase. }\end{array}$ & $\begin{array}{l}\text { Magnetite and } \\
\text { epidote. }\end{array}$ \\
\hline 101 & $\begin{array}{l}\text { Bethesda, } \\
\text { Md. }\end{array}$ & $\begin{array}{l}\text { Biotite-musco- } \\
\text { vite granite- } \\
\text { gneiss. }\end{array}$ & $\begin{array}{l}\text { Bluish } \\
\text { gray. }\end{array}$ & $\begin{array}{l}\text { Medium } \\
\text { grained. }\end{array}$ & $\begin{array}{l}\text { Microcline, quartz, } \\
\text { plagioclase, bio- } \\
\text { tite, and musco- }\end{array}$ & Apatite.............. \\
\hline 102 & $\begin{array}{l}\text { Washing- } \\
\text { ton, D. C. }\end{array}$ & Metadiorite & ... do & $\begin{array}{l}\text { Fine } \\
\text { grained. }\end{array}$ & $\begin{array}{l}\text { vite. } \\
\text { Plagioclase, quartz, } \\
\text { biotite, and horn- } \\
\text { blende. }\end{array}$ & \\
\hline 103 & $\begin{array}{c}\text { Rock Creek } \\
\text { Park, } \\
\text { Washing- } \\
\text { ton, D. C. }\end{array}$ & $\begin{array}{l}\text { Biotite-musco- } \\
\text { vite granite. }\end{array}$ & Gray....... & $\begin{array}{l}\text { Medium } \\
\text { grained. }\end{array}$ & $\begin{array}{l}\text { Microcline, quartz, } \\
\text { plagioclase, bio- } \\
\text { tite, epidote, and } \\
\text { muscovite. }\end{array}$ & $\begin{array}{l}\text { Orthoclase, apa- } \\
\text { tite, and ti- } \\
\text { tanite. }\end{array}$ \\
\hline 104 & $\begin{array}{l}\text { Granite- } \\
\text { ville, Mo. }\end{array}$ & Biotite granite.. & Red........ & .... do & $\begin{array}{l}\text { Plagioclase, micro- } \\
\text { cline orthoclase, } \\
\text { perthite, quartz, } \\
\text { and biotite. }\end{array}$ & $\begin{array}{l}\text { Biotite, chlo- } \\
\text { rite, magnet- } \\
\text { ite, hematite, } \\
\text { mus co vite, }\end{array}$ \\
\hline 105 & $\begin{array}{l}\text { Little } \\
\text { Rock, } \\
\text { Ark. }\end{array}$ & Syenite & Gray...... & $\begin{array}{l}\text { Coarse } \\
\text { grained. }\end{array}$ & $\begin{array}{l}\text { Orthoclase, biotite, } \\
\text { pyroxene, and } \\
\text { analcite. }\end{array}$ & $\begin{array}{l}\text { and fiuorite. } \\
\text { Titanite, mag- } \\
\text { netite, plagio- } \\
\text { clase, and }\end{array}$ \\
\hline 106 & $\begin{array}{c}\text { Marble } \\
\text { Falls, } \\
\text { Tex. }\end{array}$ & Biotite granite.. & Pink...... & do & $\begin{array}{l}\text { Quartz, microcline, } \\
\text { plagioclase, and } \\
\text { biotite. }\end{array}$ & $\begin{array}{l}\text { apatite. } \\
\text { Magnetite and } \\
\text { titanite. }\end{array}$ \\
\hline 107 & Kingsland, & do & ... do .... & ... do ..... & do & ... do . .... \\
\hline 108 & $\begin{array}{l}\text { Tex. } \\
\text { do }\end{array}$ & -do - & & (1) & Plo & do \\
\hline 109 & $\begin{array}{l}\text { Mountain } \\
\text { Cross, } \\
\text { Colo. }\end{array}$ & Granodiorite.... & $\begin{array}{l}\text { Bluish } \\
\text { gray. }\end{array}$ & $\begin{array}{l}\text { Fine } \\
\text { grained. }\end{array}$ & $\begin{array}{l}\text { Plagioclase, quartz, } \\
\text { bio tite, green } \\
\text { hornblende, and } \\
\text { orthoclase. }\end{array}$ & $\begin{array}{l}\text { Titanite and } \\
\text { magnetite. }\end{array}$ \\
\hline 110 & $\begin{array}{l}\text { Prescott, } \\
\text { Ariz. }\end{array}$ & do & ... do & ..... do & $\begin{array}{l}\text { Plagioclase, quartz, } \\
\text { microcline, a n d } \\
\text { biotite. }\end{array}$ & $\begin{array}{l}\text { Magnetite, apa- } \\
\text { tite, augite, }\end{array}$ \\
\hline 111 & $\begin{array}{l}\text { Columbia } \\
\text { National } \\
\text { Forest, } \\
\text { Wash. }\end{array}$ & $\begin{array}{l}\text { Basalt } \\
\text { porphyry. }\end{array}$ & $\begin{array}{l}\text { Nearly } \\
\text { black. }\end{array}$ & ..... do ....... & $\begin{array}{l}\text { High-iron glass and } \\
\text { plagioclase. }\end{array}$ & $\begin{array}{l}\text { Pyroxene and } \\
\text { magnetite. }\end{array}$ \\
\hline
\end{tabular}

See footnotes at end of table. 


\section{and their uses-Continued}

$\mathrm{HCl}$ and 9 parts of water. Physical properties in column 10 are denoted as follows: $a=$ compressive strength, The signs "+" following letters denote that the results of tests were 10 percent or more above average and of average. Test values for the various samples are given in table 3 and figure 8.]

\begin{tabular}{|c|c|c|c|c|c|}
\hline Mineral alterations & 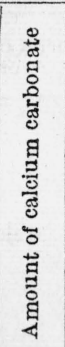 & $\begin{array}{l}\text { Physical char- } \\
\text { acteristics }\end{array}$ & Main uses & 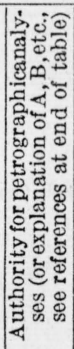 & $\begin{array}{l}\text { Remarks and year } \\
\text { production started }\end{array}$ \\
\hline (8) & (9) & (10 & (11) & (12) & (13) \\
\hline $\begin{array}{l}\text { Very small amount of } \\
\text { secondary magnetite } \\
\text { and chlorite. } \\
\text { Feldspar slightly altered } \\
\text { to sericite or kaolin. }\end{array}$ & $\begin{array}{l}\text { Per- } \\
\text { cent }\end{array}$ & $\begin{array}{l}a+, b, c-, d-\ldots \\
a+, c-\ldots\end{array}$ & Monumental. & B & \\
\hline $\begin{array}{l}\text { Secondary chlorite and } \\
\text { calcite. }\end{array}$ & 0.36 & $a, b, c-, d-\ldots$ & $\begin{array}{l}\text { Architectural and } \\
\text { constructional. } 46\end{array}$ & $\mathrm{H}$ & (1816). \\
\hline $\begin{array}{l}\text { Feldspars considerably } \\
\text { altered to sericite. }\end{array}$ & -..... & $\begin{array}{l}a-, b, c+, d- \\
a-, c+\end{array}$ & Constructional........ & $\underset{\mathrm{B}}{\mathrm{H}}$ & $\begin{array}{l}\text { The microcline forms } \\
\text { large eyes about } \\
\text { which lamellar min- } \\
\text { erals are bent. } \\
\text { Do. }\end{array}$ \\
\hline $\begin{array}{l}\text { Plagioclase extensively } \\
\text { altered to sericite; bio- } \\
\text { tite partly to chlorite. }\end{array}$ & (n) & $a-, c-$ & Monumental. & B & $\begin{array}{l}\text { Material no longer in } \\
\text { production. }\end{array}$ \\
\hline Secondary zoisite........ & & ...... do . . & Rubble masonry .... & I & \\
\hline $\begin{array}{l}\text { Microcline and plagio- } \\
\text { clase slightly weath- } \\
\text { ered to sericite. }\end{array}$ & & $a-, b+, c+\ldots$ & .... do . . . & B & $\begin{array}{l}\text { Material has a gneiss- } \\
\text { ic structure. }\end{array}$ \\
\hline $\begin{array}{l}\text { Secondary kaolin and } \\
\text { magnetite. }\end{array}$ & $\ldots$ & $a+, b-, c-\ldots$ & $\underset{\text { monumental. }}{\text { Architectural }}$ and & $J$ & \\
\hline $\begin{array}{l}\text { Orthoclase extensively } \\
\text { altered to apatite and } \\
\text { analcite. }\end{array}$ & 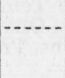 & $a-, b+, c+\ldots$ & $\begin{array}{l}\text { Architectural and } \\
\text { constructional. }\end{array}$ & B & $\begin{array}{l}\text { Stone has a poikilitic } \\
\text { texture. }\end{array}$ \\
\hline $\begin{array}{l}\text { Microcline slightly al- } \\
\text { tered to sericite. Bio- } \\
\text { tite somewhat chlori- } \\
\text { tized. }\end{array}$ & .56 & $\underset{e-}{a-}, c-, d-$ & do..$^{48} \ldots$ & B & \\
\hline $\begin{array}{l}\text { Plagioclase slightly al- } \\
\text { tered to calcite, fibrous } \\
\text { quartz, and sericite. }\end{array}$ & .63 & $\begin{array}{l}a-, b, c-, d- \\
a+, b-, c-\end{array}$ & ............ & B & $\begin{array}{l}\text { The material has a per- } \\
\text { ceptible gneissic } \\
\text { structure. }\end{array}$ \\
\hline $\begin{array}{l}\text { Marked alteration of } \\
\text { plagioclase to calcite, } \\
\text { sericite, and epidote. }\end{array}$ & .85 & $\begin{array}{l}a-, c \ldots . . \\
a+, b-, c-, d-\end{array}$ & & B & $\begin{array}{l}\text { Porphyritic feldspars } \\
\text { usually less than } 18 \\
\text { in. in size. }\end{array}$ \\
\hline
\end{tabular}


TABLE 1.-Description of granites

[Calcium carbonate in column 9 was determined from that part of the sample which was soluble in 1 part of $b=$ porosity, $c=$ absorption, $d=$ water transmission rate, $e=$ deterioration in the soaking and drying test. "-" 10 percent or more below average. When no sign follows a letter, the results were within 10 percent

\begin{tabular}{|c|c|c|c|c|c|c|}
\hline & \multirow[b]{2}{*}{ Source } & \multirow[b]{2}{*}{ Classification } & \multirow[b]{2}{*}{ Color } & \multirow[b]{2}{*}{ Texture } & \multicolumn{2}{|c|}{$\begin{array}{l}\text { Mineral constituents in descending } \\
\text { order of abundance }\end{array}$} \\
\hline 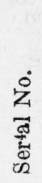 & & & & & Major & Minor \\
\hline (1) & (2) & (3) & (4) & (5) & (6) & ( $\boldsymbol{\gamma})$ \\
\hline 112 & $\begin{array}{l}\text { Hillsboro, } \\
\text { Oreg. }\end{array}$ & Augite-diorite... & $\begin{array}{l}\text { Greenish } \\
\text { gray. }\end{array}$ & $\begin{array}{l}\text { Medium } \\
\text { grained. }\end{array}$ & $\begin{array}{l}\text { Plagioclase (ande- } \\
\text { sine) and pyrox- } \\
\text { ene. }\end{array}$ & Magnetite . \\
\hline 113 & $\begin{array}{l}\text { San Fran- } \\
\text { cisco, } \\
\text { Calif. }\end{array}$ & $\begin{array}{l}\text { Biotite-musco- } \\
\text { vite granite. }\end{array}$ & $\begin{array}{l}\text { Light } \\
\text { gray. } \\
\text { do }\end{array}$ & $\begin{array}{l}\text { Medium to } \\
\text { fine } \\
\text { grained. }\end{array}$ & $\begin{array}{l}\text { Microcline, quartz, } \\
\text { plagioclase, bio- } \\
\text { tite, and musco- } \\
\text { vite. }\end{array}$ & \\
\hline 115 & $\begin{array}{l}\text { Alhambra, } \\
\text { Calif. }\end{array}$ & $\begin{array}{l}\text { Hypersthene } \\
\text { gabbro. }\end{array}$ & $\begin{array}{l}\text { Dark } \\
\text { gray. }\end{array}$ & $\begin{array}{l}\text { Fine } \\
\text { grained. }\end{array}$ & $\begin{array}{l}\text { Plagioclase, pyrox- } \\
\text { ene, and horn- } \\
\text { blende. }\end{array}$ & $\begin{array}{l}\text { Magnetite and } \\
\text { biotite. }\end{array}$ \\
\hline 116 & ............ & do & . do do _...... & .... do & do & - do do \\
\hline
\end{tabular}

A. Dale, the Commercial Granites of New England, U. S. Geol. Survey Bul. 738 (1923).

B. Insley and Tregoning, National Bureau of Standards (1939).

C. Watson, Granites of the Southeastern Atlantic States, U. S. Geol. Survey Bul. 426 (1910).

D. Buckley, Building and Ornamental Stones of Wisconsin, Bul. IV, Wisconsin Geol. Survey (1898). E. Bowles, Structural and Ornamental Stones of Minnesota, U. S. Geol. Survey Bul. 663 (1918).

F. Thiel and Dutton, The Architectural, Structural, and Monumental Stones of Minnesota, Bul. 25, Minn. Geol. Survey (1935).

G. Newland, The Quarry Materials of Now York, Bul. 181, New York State Museum (1916).

$\mathrm{H}$. Merrill and Mathews, The building and decorative stones of Maryland, vol. 2, Maryland Geol. Survey (1898).

I. U. S. Geol. Survey (1917).

J. Buckley and Buehler, The quarry industry of Missouri, vol. 2, 2d series, Missouri Bureau of Mines and Geology (1904).

K. Tarr and Neuman, A Study of the Effects of Heat on the Missouri Granites, Engineering Experiment Station Series 14, University of Missouri (1914).

1 New York Customhouse; Chicago and Northwestern Railway Station, Chicago, Ill.; State Savings Bank, Lansing, Mich.; West Street Building and United States Realty Building, New York, N. Y.; piers for Hell Gate Bridge and large columns in St. John's Cathedral in New York City.

2 Post Office Department Building (old), Washington, D. C.; Masonic Temple, Philadelphia, Pa.; Savings Bank, Wilmington, Del; Board of Trade Building, Chicago, Ill.; Post Office and Customhouse, Brooklyn, N. Y.; Manhattan Bank, New York City; General Wood's Monument, Troy, N. Y.; part of New York Customhouse.

${ }_{3}$ United States Mint, Philadelphia, Pa.; basement of New York Customhouse; Brooklyn anchorage of Manhattan bridge; basement of House Office Building (new), Washington, D. C.; Lafayette High School, Brooklyn, N. Y.; interior of St. John's Cathedral, New York City.

4 Customhouse, Buffalo, N. Y.; Customhouse, Fall River, Mass.; Western Savings Bank, Philadelphia, Pa.; National Bank of Commerce, Kansas City, Mo.; Bellevue Hospital, New York City; Winter Mausoleum, Pittsburgh, Pa.; Kress Mausoleum, Allentown, $\mathrm{Pa}$.

${ }_{3}$ Post Office, Albany, N. Y.; Bates Building, Philadelphia, Pa.; part of the Saratoga Monument, Saratoga, N. Y.

City Hall, Portland, Maine; C \& N Railway and Field Annex buildings, Chicago, Ill.; Union Trust Building, South Bend, Ind.; Exchange National Bank, Tulsa, Okla.; Penn Mutual Life Building, Philadelphia. Pa.; General Grant's Tomb, New York City; Hahnemann Monument, Washington, D. C.

7 Post Office, Lowell, Mass.; Museum of Fine Arts, Boston, Mass.; Ninth Regiment Armory, New York City; National Security Bank, Los Angeles, Calif.; Stevens Hotel (base), Chicago. Ill.; Insurance Company of North America building. Philadelphia, Pa.: South Office Building of the State Capitol, Harrisburg, Pa.; New York State Office Building, Albany, N. Y.; Pioneer Monument, Harrodsburg, Ky. 


\section{and their uses-Continued}

$\mathrm{HCl}$ and 9 parts of water. Physical properties in column 10 are denoted as follows: $a=$ compressive strength The signs " +" following letters denote that the results of tests were 10 percent or more above average and of average. Test values for the various samples are given in table 3 and figure 8.]

\begin{tabular}{|c|c|c|c|c|c|}
\hline Mineral alterations & 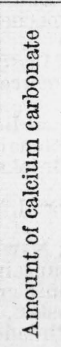 & $\begin{array}{l}\text { Physical char- } \\
\text { acteristics }\end{array}$ & Main uses & 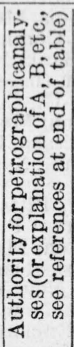 & $\begin{array}{l}\text { Remarks and year } \\
\text { production started }\end{array}$ \\
\hline (8) & (9) & (10) & (11) & (12) & (13) \\
\hline $\begin{array}{l}\text { Plagioclase considerably } \\
\text { altered to albite, cal- } \\
\text { cite and sericite; pyrox- } \\
\text { ene to uralite and antig- } \\
\text { orite. } \\
\text { Plagioclase sometimes } \\
\text { altered to sericite and } \\
\text { calcite. }\end{array}$ & $\begin{array}{l}\text { Per- } \\
\text { cent } \\
5.20\end{array}$ & $\begin{array}{l}a+, b+, c \ldots . . \\
a-, b+, c+\ldots .\end{array}$ & $\begin{array}{l}\text { Architectural and } \\
\text { constructional. }\end{array}$ & B & $\begin{array}{l}\text { Material has a slight } \\
\text { gneissic structure. }\end{array}$ \\
\hline $\begin{array}{l}\text { Pyroxene (hypersthene) } \\
\text { slightly altered to } \\
\text { hornblende and chlo- } \\
\text { rite. }\end{array}$ & 1.51 & $a+, b-, c-, d-$ & Monumental & $\begin{array}{l}\mathrm{B} \\
\mathrm{B}\end{array}$ & \\
\hline
\end{tabular}

8 Post Office buildings at Indianapolis, Ind. (base), Cleveland, Ohio, Milwaukee, Wis., and Jersey City, N. J.; New York City Public Library (base); St. Louis Public Library; United States Mint, Philadelphia, $\mathrm{Pa}$.

Post Office buildings at Buffalo, N. Y., and Hartford, Conn.; Standard Oil Building, New York City.

${ }^{10}$ Lyman Allyn Museum, New London, Conn.; Christian Science Publishing Building, Boston, Mass.

Douglas Memorial Hall, Washington, D. C.; United States Post Office Building, Cleveland, Ohio; Liberty Mutual Insurance Building, Boston, Mass.; Southern New England Telephone Building, New Haven, Conn. All of these were erected between 1930 and 1938.

${ }_{11}$ Christian Science Church, Boston, Mass.; Lehigh County Court House, Allentown, Pa.; MedicoChirugical Hospital, Philadelphia, Pa.; Post Offices at Dayton, Ohio, Lincoln, Nebr., Staten Island, N.Y., and Newark, N. J.; War Memorial, Indianapolis, Ind.; Gulf Building, Pittsburgh, Pa.; First National Bank, New York City; Rackham Memorial, Ann Arbor, Mich.

12 First National Bank, Chicago, Ill.; First National Bank, Cincinnati, Ohio; Wisconsin Telephone Building, Milwaukee, Wis.; Franklin Savings Bank, New York City; City Hall, Lowell, Mass.; State Library, Concord, N. H.; Masonic Memorial Temple, Alexandria, Va.

13 Union Arcade Building, Pittsburgh, Pa.; Fidelity Mutual Life Insurance Building, Philadelphia Pa.; Post Office (polished columns), Washington, D. C.; Dime Savings Bank (exterior columns), Detroit, Mich.; Missouri State Capitol (interior columns), Jefferson City; First National Bank, Bridgeport, Conn. (exterior columns); Baxter Building, Portland, Maine.

14 Pier No. 4 of Haverhill, Mass., bridge; trimmings of Majestic Theater, Chicago, Ill.; Ferguson Mausoleum, Kensico Cemetery, New York, N. Y.

15 Pylon Monuments of Manhattan Bridge, Brooklyn Plaza, N. Y.; L. C. Smith Mausoleum, Syracuse N. Y.; C. W. Post Mausoleum, Battle Creek, Mich.

i6 War Memorial, Springfield, Mass. (1933); Philip B. Armour Monument, Chicago, Ill. (1918); Statue of Youth, Barre, Vt. (1923); A very Hopwood Monument, Cleveland, Ohio (1928); Navy and Marine Memorial, Newport, R. I. (1938); Richard Bartholdt Monument, St. Louis, Mo. (1936).

${ }_{17}$ Iowa Soldiers' Monument, Chattanooga, Tenn.; Pennsylvania Soldiers' Monument, York, Pa.; Hearn Monument, Woodlawn, N. Y.; Rouse Mausoleum, Winchester, Va.; Soldiers and Sailors Monument, Angola, Ind.

${ }_{18}$ Prison-ship Martyrs' Monument in Fort Greene Park, Brooklyn, N. Y.

io Northwestern Mutual Life Building, Milwaukee, Wis.; Museum of Fine Arts, Minneapolis, Minn.; Soldiers' and Sailors' Memorial, Wichita, Kans.; Bridgeport 'Trust Building, Bridgeport, Conn.

${ }_{20}$ Pennsylvania State Capitol, Harrisburg, Pa.; Cook County Court House, Chicago, Ill.; City Hall Cleveland, Ohio; Mercantile Trust Building, St. Louis, Mo.; Kentucky State Capitol (base and 36 polished columns), Frankfort, Ky.; Navy Memorial, Vicksburg, Miss.; Memorial Arch, Port Huron, Mich.

${ }_{21}$ Columbia University, New York City (16 polished columns); General Gomez monument in Cuba; Die in Bennington monument, Bennington, Vt.; Bank of Montreal (34 columns); W. C. T. U. fountain. Orange, Mass. (columns and die).

(Footnotes continued on p. 180) 
(Footnotes continued from p. 179)

22 Post Office, Troy, N. Y.; Diamond Bank, Pittsburgh, Pa.; McFadden Building, Chicago, Ill.; Royal Baking Powder Building, New York City.

${ }_{23}$ First National Bank Building, New York City (1932).

14 Finance Building, State Capitol, Harrisburg. Pa.; New Jersey State Office Building, Trenton, N. J.; Massachusetts State House (wings), Boston, Mass.; Fidelity Mutual Life Building, Philadelphia, Pa.; Masonic Temple, St. Louis, Mo.; New Orleans Post Office (base and approaches) New Orleans, La.; County Court House, Hartford, Conn.

${ }^{25}$ Hanover National Bank, New York City; Public Library, Boston. Mass.; Public Library, Columbus, Ohio; National Museum (base), Washington, D. C.; Chamber of Commerce, Rochester, N. Y.; Customhouse, Wilmington, N. C.; Commercial National Bank, Chicago, Ill.

${ }^{26}$ Customhouse, Boston; Masonic Temple, Chicago. Ill.; Union National Bank, Pittsburgh, $\mathrm{Pa}$.

si Hamilton Club, Chicago, Ill.; Barnes Building, Wichita, Kans.; National State Bank, Newark, N. J.;

Post Office, Elkhart, Ind.; Syndicate Building, Minneapolis, Minn.; Brooks Bros. Building, New York City.

${ }_{28}$ City Deposit Bank, Pittsburgh, Pa.; Soldiers' and Sailors' Memorial, Syracuse, N. Y.; Alexander Hamilton Memorial, Grant Park, Chicago, Ill.

20 South Station, Boston, Mass.; Bessemer Building, Pittsburgh, Pa.; Newberry Library, Chicago, III Battle Monument (polished columns), West Point, N, Y.; McMillan fountain, Washington, D. C.; Sheridan Monument (base), New York City; Hooker Monument (State House grounds), Boston, Mass.

${ }_{30}$ Telephone Building, New York City; South Terminal Station, Boston, Mass.; Grosvenor Building, Providence, R. I.; New England Telephone Building, Hartford, Conn.; Philadelphia Savings Fund Society, Philadelphia, Pa.; Provident Savings Bank, Baltimore, Md.; Rochester Savings Bank, Rochester, N. Y.; Fulton Building, Pittsburgh, Pa.; Dixie Terminal Building, Cincinnati, Ohio; Whitney Central Bank, New Orleans, La.

${ }_{31}$ Union Trust Co. (1906) and Arlington Bridge (1917), Washington, D. C.; Guilford County Courthouse (1917), Greenshoro, N. C.; State Office Building (1938), Raleigh, N. C.; United States Depository (1936), Fort Knox, Ky.; Wright Brothers Memorial (1932), Kitty Hawk, N. C.; Rose Hill Mausoleum (1925), Rose Hill Cemetery, Chicago, Ill.

${ }_{32}$ Raleigh, N. C., Post Office (1875) and addition to same (1937); Governor Endicott Monument and Statue (1937), Boston, Mass.; Antietam Creek Bridge (1936) near Hagerstown, Md.

${ }_{33}$ Fidelity Trust Building, Philadelphia, Pa.; Blackstone Hotel, Chicago, Ill.; Butler University, Indianapolis, Ind.; President Buchanan Memorial, Washington, D. C.; Morris Mausoleum, Cleveland, Ohio; Sutherland Exhedra, Greenwood Cemetery, Brooklyn, N. Y.; World War Memorial, Des Moines, Iowa: Lew is and Clark Memorial, Charlottesville, Va.

${ }^{34}$ Field office. George Washíngton Bridge, Fort Lee, N. J.; High School, Asheville. N. C.; Hilton Park way bridges, Baltimore, Md.; Post Office, Wilmington, Del.; Howard University power plant and Chemistry Building, Washington, D. C.; Loyola University, Chicago, Ill.; Beardsley Monument, Indianapolis, Ind.

${ }_{35}$ United States Post Office and Court House buildings at Wilmington, N. C., and Asheville, N. C.: Post Office buildings at Charleston, S. C., Durham, N. C., Chillicothe, Ohio, Traverse City, Mich., Florence, S. C., and Charlottesville, Va.; Hussey Building, Pittsburgh, Pa.; New Land and Trust Building, Philadelphia, Pa.; United States Customhouse, Baltimore, Md.; Empire Bank Building, Clarksburg, W. Va.

${ }_{38}$ Customhouse, Mobile. Ala.; Customhouse Extension, Denver, Colo.; Supreme Court Building, Chey. enne, W yo.; Veterans' Administration Facility, Columbia, S. C. Central Office "U" Building, Detroit, Mich.; Post Office, Provo, Utah; Post Office Garage, Chicago, Ill.; Frankford Arsenal, Philadelphia, Pa. State Office, Oklahoma City, Okla.; Southern Bell Telephone Building, Charleston, S. C.; P Street Bridge, Washington, D. C.

${ }_{37}$ General Grant Sarcophagus in Grant's Tomb, New York City; Illinois State Monuments, Andersonville, Ga.

${ }_{38}$ State Office Building, St. Paul, Minn.; City Hall (1927), Duluth, Minn.; Gas and Electric Building (1925), Oklahoma City, Okla.: Ford Museum (1929), Dearhorn, Mich.; Merchants Mart (1929), Chicago. Ill.; Jefferson County Courthouse (1929), Birmingham, Ala.; Criminal Court Building (1928), New Orleans, La.; Cadillac Building (1926), Boston, Mass.; New York Central Station (1926), Buffalo, N. Y.; Gallinger Hospital, (1925), Washington, D. C.: Koppers Building (1927), Pittsburgh, Pa.; City Hall (1926), Columbus, Ohio: Chamber of Commerce (1935), Jacksonville, Fla.; Telephone Building (1925), Dallas, Tex.; First National Bank (1931), San Jose, Calif.; Young's Market (1922), Los Angeles, Calif.; Schroeder Hotel (1928), Milwankee, Wis.; Post Office (1926), Des Moines, Iowa.

${ }_{39}$ Coliseum (1936), Fort Worth, Tex.; County Court House (1936), Oklahoma City, Okla.; Post Office (1936), . J acksonville, Ill.; Marine Hospital (1937), St. Louis, Mo.; State Capitol (1930), Baton Rouge, La.; Telephone Building, Evansville, Ind.; Price Building, Kansas City, Mo.; County Court House (1938), Jackson ville, Miss.; Capitol Club Office Building, Raleigh, N. C.

${ }_{40}$ The Cathedral, St. Paul, Minn.; Tribune Tower (base), Chicago. Ill.; United States Court House (1934), Foley Square. New York City; Capitol Plaza Fountain (1931), Washington, D. C.; addition to Milwaukee Post Office (1930); Post Office (1928), Duluth, Minn.; Book Cadillac Hotel, Detroit, Mich.; Justice Building, St. Louis, Mo.; New City Hall, Kansas City, Mo.;Telephone Building, Oklahoma City, Okla.

${ }_{41}$ Telephone Building (1936), St. Paul, Minn.; Vanderbilt Monument (1936), New York City; Esquire Theatre (1937), Chicago, Ill.; Dunning Mausoleum (1937), Fort Worth. Tex.; Post Office (1937), Los Angeles. Calif.; Telephone Building (1931), Cincinnati, Ohio; Adler Planetarium (1928), Chicago, Ill.: David Stott Building (1925), Detroit, Mich.; Mariner Tower Building (1930), Milwaukee. Wis.

42 Post Office Building (1868), St. Paul, Minn.

43 Post Office, St. Cloud, Minn. (1902): base of old Minneapolis Post Office (1884).

44 St. John's Cathedral (interior), residence of Charles M. Schwab, Postal Telegraph Building, Cross Building, and Bronx Geological Gardens, New York City; Post Office, Bangor, Maine; Connecticut State Armory, Hartford, Conn.

${ }_{45}$ American Museum of Natural History, New York City, National Bank of Clayton, N. Y.; Maryland Museum Building, Baltimore, Md. (polished columns).

${ }_{46}$ Fort McHenry (part of walls), Baltimore harbor, Md.; St. Dominick Church, Washington, D. C.

47 Washington University, St. Louis, Mo.; State House, Springfield, Ill.; Paxton Building, Omaha, Nebr.; Marshall Field Building. Chicago, Ill.; Whitney Building, New Orleans, La.; Art Museum, Cincinnati, Ohio; Keith and Perry Building, Kansas City, Mo.; Central Savings Bank. Baltimore, Md.; Society for Savings, Cleveland, Ohio; F. E. Knowles and Company Building, San Francisco, Calif.; Thos. Allen Monument, Pittsfield, Mass.

${ }_{48}$ Texas State Capitol, Austin, Tex.; and Fort Worth Court House, Fort Worth, Tex. 
The most abundant mineral in granite is generally feldspar, which is of two main types-namely, potash feldspar, a silicate of potash and alumina; and soda-lime feldspar (plagioclase), a silicate of sodium, aluminum, and calcium. Common species of the first type are orthoclase and microcline and of the second type albite, oligoclase, andesine, labradorite, bytownite, and anorthite. Both types in granite usually show distinct crystal forms, and the crystal faces when exposed at the surface cause the sparkling effect which is often pronounced in coarse-grained granite. The color of plagioclase is usually white or gray, while the potash feldspar occurs in various colors, such as gray, pink, buff, or green.

Quartz $\left(\mathrm{SiO}_{2}\right)$ in granite is sometimes nearly clear but more often clouded by inclusions of dark particles, when it is called smoky quartz. It can be identified by its vitreous luster, irregular fracture, and greater hardness than feldspar. Quartz is probably the most inert and durable mineral in granite.

In most granites mica ranks third in abundance. Black mica (biotite) is more common than white mica (muscovite), but often both species occur in the same rock. Mica is a relatively soft mineral easily identified by the fact that it can be separated into thin sheets which are flexible.

The black basic minera's, hornblende and pyroxene, occur as prominent constituents in some granites. They can be distinguished from biotite by their greater hardness and the fact that they have no definite cleavage. These minerals are complex silicates of magnesium and iron. Several species of pyroxene, such as diopside, augite, aegirite, and riebeckite, are found in granite.

A large number of other minerals occur in granite, but generally they make up a very small part of the rock. For information on them the reader may consult a textbook on mineralogy.

"Mineral alterations" (column 8 of table 1) refer to those changes which have occurred since the original minerals formed. Most alterations are attributed to weathering, and they are confined mainly to rock near the earth's surface or near open joints. Common alterations are as follows: Feldspar to kaolin or mica; biotite to chlorite; and plagioclase, in part, to calcite.

Partial chemical analyses were made on 80 samples to determine the amounts of calcite and pyrite. The results for calcite only are given because the sulfide was uniformly low and for 71 samples it was below 0.02 percent.

Several specific examples of structural use are cited in table 1, along with the dates of construction when known. For those granites which have been used extensively, only a few representative examples in different sections of the country are referred to.

\section{PHYSICAL TESTS}

\section{COMPRESSIVE STRENGTH}

The specimens (described in section II) were tested on a $300,000-1 b$ hydraulic machine with a spherical adjustment block above the specimen. Disks of hardened steel 2.5 in. in diameter by 0.5 in. in thickness were placed between the ends of the specimens and the machine plates, and as these became roughened they were resurfaced. One 
thickness of thin blotting paper was placed between the plates and the ends of the specimen in order to assure a satisfactory distribution of the load. The load was applied at a rate of $100 \mathrm{lb} / \mathrm{in} .^{2}$ per second. Crushing usually occurred suddenly with very little chipping of the corners before the final break.

Some producers have raised the point that very coarse-grained materials would give higher compressive strength if larger specimens were used. For this reason comparative tests were made on two sizes of specimens from each of five different granites selected from the coarsest grained materials of the series. The results of these comparisons are given in table 2.

TABLE 2.-Effect of size of test specimen on strength of granite

\begin{tabular}{|c|c|c|c|c|c|c|c|}
\hline \multirow{2}{*}{ Serial No. } & \multicolumn{2}{|c|}{ Size of specimen } & \multirow{2}{*}{$\begin{array}{l}\text { Number } \\
\text { of tests }\end{array}$} & \multicolumn{3}{|c|}{ Compressive strength a } & \multirow{2}{*}{$\begin{array}{l}\text { Varia- } \\
\text { tion }\end{array}$} \\
\hline & Diameter & Height & & Maximum & Minimum & Average & \\
\hline $\begin{array}{l}12 \\
12 \mathrm{a} 0 \\
13 \\
36 \\
36 \\
81 \\
107 \\
107\end{array}$ & in. $\begin{array}{r} \\
2.1 \\
3.0 \\
2.1 \\
2.5 \\
2.1 \\
2.5 \\
2.1 \\
2.5 \\
2.1 \\
2.5\end{array}$ & $\begin{array}{r}\text { in. } \\
2.5 \\
3.5 \\
2.1 \\
2.5 \\
2.5 \\
3.0 \\
2.5 \\
3.0 \\
2.5 \\
3.0\end{array}$ & $\begin{array}{l}2 \\
3 \\
6 \\
6 \\
2 \\
4 \\
2 \\
4 \\
2 \\
4\end{array}$ & $\begin{array}{l}\text { lb/in. } 2 \\
21,300 \\
26,100 \\
29,300 \\
25,700 \\
20,400 \\
25,700 \\
23,300 \\
23,200 \\
21,700 \\
23,400\end{array}$ & $\begin{array}{r}l b / i n .2 \\
17,900 \\
25,300 \\
23,800 \\
22,600 \\
18,900 \\
22,000 \\
17,300 \\
21,200 \\
16,200 \\
20,400\end{array}$ & $\begin{array}{c}l b / \text { in } .^{2} \\
19,600 \\
25,700 \\
26,700 \\
24,300 \\
19,600 \\
23,800 \\
20,300 \\
22,200 \\
19,000 \\
22,100\end{array}$ & $\left\{\begin{array}{r}\text { Percent } \\
+31 \\
-9 \\
+21 \\
+9 \\
+16\end{array}\right.$ \\
\hline
\end{tabular}

all specimens tested dry and loaded perpendicular to rift. - Computed by the formula $\frac{\left(C_{2}-C_{1}\right) 100}{C_{1}}$, in which $C_{1}$ and $C_{2}$ are, respectively, the average compressive

- Serial No. 12a was from the same quarry as No. 12 but taken at a later date.

The specimens showing greatest difference in strength between the two sizes were cut from two different samples from the same quarry (12 and 12a), and hence the comparisons may not be justified in this case. If the average values are weighted in accordance with the number of tests for each size, the weighted mean for all the larger specimens is about 4 percent higher than that for all the smaller specimens. If a similar computation is made omitting the values for 12 and $12 \mathrm{a}$, the results indicate no appreciable difference.

Results of compression tests on cylindrical specimens 2.1 by 2.5 in. are given in table 3 for 114 samples of granite. The average strength for all of the granites in the dry condition was $24,500 \mathrm{lb} / \mathrm{in} .^{2}$ and the highest recorded was $60,000 \mathrm{lb} /$ in. $^{2}$ Occasional strengths below $10,000 \mathrm{lb} /$ in. $^{2}$ were obtained for samples apparently taken near the surface of the ground. Some granites appear to be stronger when loaded perpendicular to the rift, but the grand averages of all values for each of the two directions of loading were practically the same. Many of the samples showed a reduction in strength for the wet condition, and the average of all tests on wet specimens was about 12 percent lower than on dry specimens. 
TABLE 3.-Average values obtained for compressive strength, absorption, density porosity and weight per cubic foot of various granites, by States

\begin{tabular}{|c|c|c|c|c|c|c|c|c|c|c|c|}
\hline \multirow{3}{*}{ Serial No. } & \multicolumn{4}{|c|}{$\begin{array}{l}\text { Compressive strength perpen- } \\
\text { dicular }(A) \text { and parallel }(B) \text { to } \\
\text { the rift }\end{array}$} & \multicolumn{3}{|c|}{$\begin{array}{l}\text { Absorption by weight } \\
\text { for immersion periods } \\
\text { of }\end{array}$} & \multicolumn{2}{|c|}{ Density } & \multirow{3}{*}{$\begin{array}{c}\text { Poros- } \\
\text { ity }\end{array}$} & \multirow{3}{*}{$\begin{array}{l}\text { Com- } \\
\text { puted } \\
\text { weight } \\
\text { per } \\
\text { cu. ft. }\end{array}$} \\
\hline & \multicolumn{2}{|c|}{ Specimens dry } & \multicolumn{2}{|c|}{ Specimens wet } & \multirow{2}{*}{$48 \mathrm{hr}$} & \multirow{2}{*}{14 days } & \multirow{2}{*}{$1 \mathrm{yr}$} & \multirow{2}{*}{ Bulk } & \multirow{2}{*}{ True } & & \\
\hline & $A$ & $B$ & $A$ & $B$ & & & & & & & \\
\hline
\end{tabular}

MAINE

\begin{tabular}{|c|c|c|c|c|c|c|c|c|c|c|c|}
\hline & & lb/in. ${ }^{2}$ & $l b / i n .2$ & lb/in.? & & & $\%$ & & & & \\
\hline & 27,600 & 29,200 & 26,500 & 31,800 & 0.23 & 0.25 & 0.28 & 2. 624 & 2. 657 & 1. 24 & 164 \\
\hline & $\begin{array}{l}34,800 \\
16,900\end{array}$ & $\begin{array}{l}37,000 \\
21,200\end{array}$ & $\begin{array}{l}24,100 \\
14,400\end{array}$ & $\begin{array}{l}33,400 \\
15,000\end{array}$ & $\begin{array}{l}.03 \\
.26\end{array}$ & $\begin{array}{l}.03 \\
32\end{array}$ & $\begin{array}{l}.04 \\
.048\end{array}$ & $\begin{array}{l}\text { 2. } \\
\text { 2. }\end{array}$ & $\begin{array}{l}2.976 \\
2.658\end{array}$ & $\begin{array}{r}.40 \\
1.47\end{array}$ & $\begin{array}{l}185 \\
163\end{array}$ \\
\hline & 19,400 & 26,300 & 27,300 & 25,100 & .20 & .25 & .31 & 2. 628 & 2.655 & 1. 02 & $\begin{array}{l}160 \\
164\end{array}$ \\
\hline & 28,000 & 29,700 & 27,800 & 33,800 & .15 & .18 & .21 & 2. 637 & 2. 662 & & 164 \\
\hline & 33,000 & 26,400 & 15,600 & 16,700 & .21 & .28 & .35 & 2. 605 & 2.645 & 1. 51 & $\begin{array}{l}165 \\
162\end{array}$ \\
\hline & 38,800 & 35,300 & 33,000 & 31,600 & .23 & .28 & .34 & 2. 611 & 2. 642 & 1.14 & 163 \\
\hline & $\begin{array}{r}22,800 \\
2 ? 0\end{array}$ & $\begin{array}{r}23,200 \\
{ }_{3}^{2},\end{array}$ & $\begin{array}{r}23,000 \\
17\end{array}$ & $\begin{array}{l}21,900 \\
17\end{array}$ & $\cdot 23$ & .31 & $\begin{array}{r}.47 \\
20\end{array}$ & 2. 602 & $\begin{array}{l}2.644 \\
2.682\end{array}$ & 2. 00 & 162 \\
\hline & 22,900 & ${ }^{23}, 600$ & 24,400 & 17,300 & $\cdot 22$ & $\begin{array}{l}.25 \\
36\end{array}$ & $\begin{array}{r}29 \\
39\end{array}$ & $\begin{array}{l}2.660 \\
2.623\end{array}$ & 2.682 & $1_{32}^{83}$ & $\begin{array}{l}166 \\
164\end{array}$ \\
\hline & 19,600 & 21, 300 & 2280 & 19,900 & $v_{22}^{10}$ & 29 & 40 & 2. 610 & & 1.36 & $\begin{array}{l}164 \\
163\end{array}$ \\
\hline & 26,700 & 28,500 & 22,600 & 24,200 & .32 & .36 & .41 & 2. 623 & 2. 659 & 1. 36 & $\begin{array}{l}165 \\
164\end{array}$ \\
\hline & 18,700 & & & & & & & & & & 164 \\
\hline & 26,000 & 20,600 & 20,300 & 21,400 & .32 & .38 & .44 & 2. 602 & 2. 641 & 1. 48 & 162 \\
\hline
\end{tabular}

NEW HAMPSHIRE

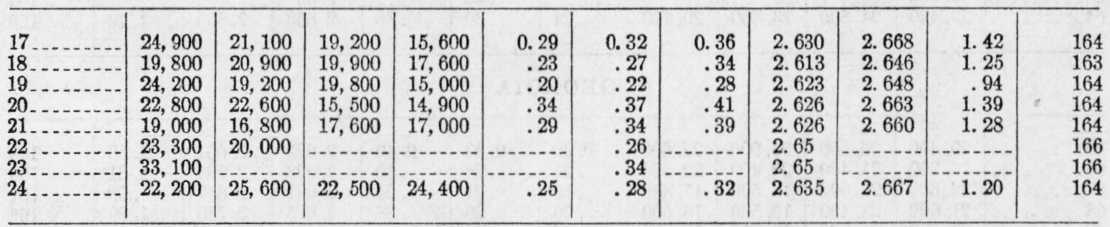

VERMONT

\begin{tabular}{|c|c|c|c|c|c|c|c|c|c|c|c|}
\hline $25 \ldots \ldots$ & 15,600 & 14,400 & 14,200 & 16,900 & 0.20 & 0.22 & 0.26 & 2. 660 & 2. 686 & 0.97 & 166 \\
\hline 27 & 18,600 & 20,600 & 15,500 & 14,200 & .26 & $\begin{array}{l}.28 \\
.28\end{array}$ & .34 & $\begin{array}{l}2.04 \\
\text { 2. } 646\end{array}$ & 2. 678 & 1. 27 & $\begin{array}{l}100 \\
165\end{array}$ \\
\hline 28 & 29,800 & 29,100 & 24,600 & 25,500 & .27 & .31 & .41 & 2. 628 & 2. 675 & 1. 76 & 164 \\
\hline & 13,800 & 13,000 & 14,200 & 14,600 & 24 & .27 & .30 & 2. 661 & 2. 695 & 1. 26 & 166 \\
\hline 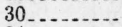 & 26,400 & 35,500 & 28,000 & 27,300 & .14 & .15 & .16 & 2. 644 & 2. 672 & 1.05 & 165 \\
\hline $31 \ldots$ & 22,300 & 19,700 & 20,400 & 17,300 & .24 & .28 & -....... & 2. 67 & - & -........... & 167 \\
\hline $32 \ldots \ldots$ & 26,700 & -........ & 20,600 & - n & - n. & .29 & $\ldots$ & 2.63 & - n. & $\ldots$ & 164 \\
\hline
\end{tabular}

MASSACHUSETTS

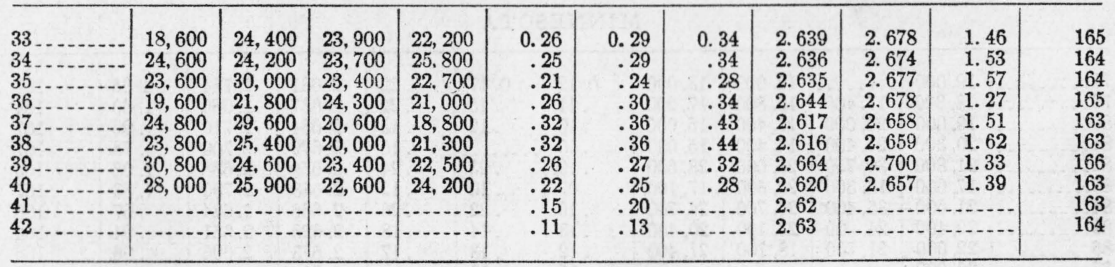

CONNECTICUT

\begin{tabular}{|c|c|c|c|c|c|c|c|c|c|c|c|}
\hline $\begin{array}{l}43 \\
44 \\
45 \\
46 \\
47\end{array} 4$ & $\begin{array}{l}16,800 \\
22,400 \\
30,800 \\
24,200 \\
23,800 \\
20,000\end{array}$ & $\begin{array}{l}19,800 \\
23,800 \\
26,800 \\
32,400 \\
26,900 \\
24,000\end{array}$ & $\begin{array}{l}21,300 \\
22,600 \\
25,600 \\
15,900 \\
22,000 \\
14,200\end{array}$ & $\begin{array}{l}22,000 \\
21,600 \\
30,000 \\
21,000 \\
24,700 \\
14,400\end{array}$ & $\begin{array}{l}0.21 \\
.19 \\
.22 \\
.31 \\
.27 \\
.18\end{array}$ & $\begin{array}{r}0.23 \\
.22 \\
.26 \\
.34 \\
.31 \\
.20\end{array}$ & $\begin{array}{r}0.28 \\
.26 \\
.34 \\
.48 \\
.40 \\
.28\end{array}$ & $\begin{array}{l}2.622 \\
2.625 \\
2.626 \\
2.617 \\
2.614 \\
2.634\end{array}$ & $\begin{array}{l}\text { 2. } 651 \\
2.657 \\
\text { 2. } 666 \\
\text { 2. } 663 \\
\text { 2. } 646 \\
\text { 2. } 662\end{array}$ & $\begin{array}{l}1.09 \\
1.21 \\
1.50 \\
1.73 \\
1.21 \\
1.05\end{array}$ & $\begin{array}{l}163 \\
164 \\
164 \\
163 \\
163 \\
164\end{array}$ \\
\hline
\end{tabular}


TABLE 3.-Average values obtained for compressive strength, absorption, density, porosity and weight per cubic foot of various granites, by States-Continued

\begin{tabular}{|c|c|c|c|c|c|c|c|c|c|c|c|}
\hline \multirow{3}{*}{ Serial No. } & \multicolumn{4}{|c|}{$\begin{array}{l}\text { Compressive strength perpen- } \\
\text { dicular }(A) \text { and parallel }(B) \text { to } \\
\text { the rift }\end{array}$} & \multicolumn{3}{|c|}{$\begin{array}{l}\text { Absorption by weight } \\
\text { for immersion periods } \\
\text { of }\end{array}$} & \multicolumn{2}{|c|}{ Density } & \multirow{3}{*}{$\begin{array}{l}\text { Poros- } \\
\text { ity a }\end{array}$} & \multirow{3}{*}{$\begin{array}{c}\text { Com- } \\
\text { puted } \\
\text { weight } \\
\text { per } \\
\text { cu.ft. }\end{array}$} \\
\hline & \multicolumn{2}{|c|}{ Specimens dry } & \multicolumn{2}{|c|}{ Specimens wet } & \multirow{2}{*}{$48 \mathrm{hr}$} & \multirow{2}{*}{14 days } & \multirow{2}{*}{$1 \mathrm{yr}$} & \multirow{2}{*}{ Bulk } & \multirow{2}{*}{ True } & & \\
\hline & $A$ & $B$ & $A$ & $B$ & & & & & & & \\
\hline
\end{tabular}

\section{NORTH CAROLINA}

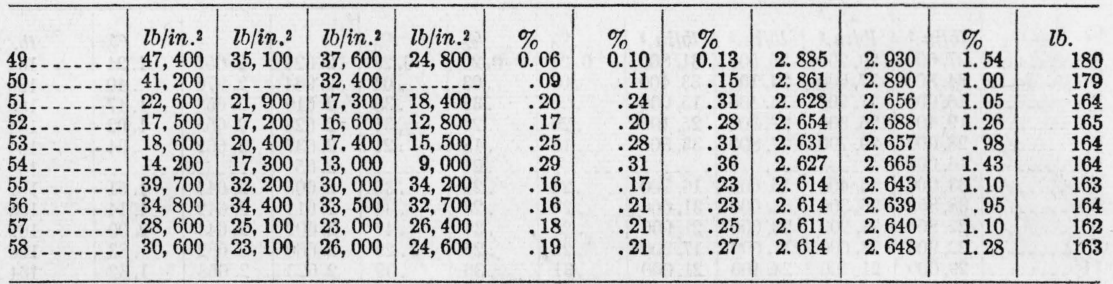

SOUTH CAROLINA

\begin{tabular}{|c|c|c|c|c|c|c|c|c|c|c|c|}
\hline $59 \ldots \ldots$ & 22,600 & 19,600 & 22,800 & 20,800 & 0.25 & 0. 28 & 0.40 & 2. 637 & 2.673 & 1.35 & 164 \\
\hline 61 & 33,200 & 34,200 & 27,800 & 26,000 & .26 & .29 & 38 & 2. 632 & 2.692 & 2.23 & 164 \\
\hline $62 \ldots \ldots$ & 27,300 & 23,500 & 27,800 & 28,300 & .22 & .23 & .30 & 2. 636 & 2. 670 & 1.27 & 164 \\
\hline 63 & $\begin{array}{l}30,700 \\
29,400\end{array}$ & 24,600 & 23,800 & 20,400 & .24 & $\begin{array}{l}.32 \\
.30\end{array}$ & .36 & $\begin{array}{l}2.63 \\
2.663\end{array}$ & 2.695 & 1.19 & $\begin{array}{l}104 \\
166\end{array}$ \\
\hline
\end{tabular}

\section{GEORGIA}

\begin{tabular}{|c|c|c|c|c|c|c|c|c|c|c|c|}
\hline $\begin{array}{l}65 \\
66 \\
67 \\
68 \\
69 \\
70\end{array}$ & $\begin{array}{l}29,400 \\
26,300 \\
22,600 \\
21,000 \\
27,800 \\
22,700\end{array}$ & $\begin{array}{l}26,500 \\
21,400 \\
25,000 \\
23,400 \\
24,000 \\
25,600\end{array}$ & $\begin{array}{l}24,900 \\
25,400 \\
18,500 \\
15,500 \\
20,800 \\
20,800\end{array}$ & $\begin{array}{l}23,600 \\
22,500 \\
17,800 \\
16,500 \\
19,800 \\
22,400\end{array}$ & $\begin{array}{l}0.30 \\
.24 \\
.19 \\
.26 \\
.22 \\
.19\end{array}$ & $\begin{array}{r}0.33 \\
.26 \\
.21 \\
.29 \\
.24 \\
.22\end{array}$ & $\begin{array}{r}0.40 \\
.30 \\
.24 \\
.36 \\
- \\
\end{array}$ & $\begin{array}{l}\text { 2. } 622 \\
\text { 2. } 628 \\
2.631 \\
2.675 \\
2.623 \\
2.634\end{array}$ & $\begin{array}{l}2.662 \\
2.663 \\
2.657 \\
2.710 \\
2.648 \\
2.660\end{array}$ & $\begin{array}{r}1.50 \\
1.31 \\
.98 \\
1.29 \\
.95 \\
.98\end{array}$ & $\begin{array}{l}163 \\
164 \\
164 \\
166 \\
162 \\
164\end{array}$ \\
\hline
\end{tabular}

\section{WISCONSIN}

\begin{tabular}{|c|c|c|c|c|c|c|c|c|c|c|c|}
\hline $\begin{array}{l}71 \\
72 \\
73 \\
74 \\
75 \\
76 \\
77\end{array}$ & $\begin{array}{l}13,800 \\
16,900 \\
27,900 \\
37,000 \\
22,700 \\
29,500 \\
31,200\end{array}$ & $\begin{array}{l}14,400 \\
20,200 \\
32,100 \\
30,600 \\
18,900 \\
42,400 \\
30,400\end{array}$ & $\begin{array}{l}17,400 \\
14,700 \\
30,800 \\
31,700 \\
16,800 \\
27,800 \\
25,800\end{array}$ & $\begin{array}{l}16,800 \\
15,000 \\
26,200 \\
31,200 \\
15,400 \\
34,200 \\
28,200\end{array}$ & $\begin{array}{l}0.05 \\
.30 \\
.07 \\
.12 \\
.10 \\
.05 \\
.08\end{array}$ & $\begin{array}{r}0.05 \\
.37 \\
.08 \\
.15 \\
.12 \\
.05 \\
.08\end{array}$ & $\begin{array}{r}0.06 \\
.46 \\
.09 \\
.15 \\
.14 \\
.06 \\
.10\end{array}$ & $\begin{array}{l}2.911 \\
2.662 \\
2.671 \\
2.624 \\
2.628 \\
2.629 \\
2.616\end{array}$ & $\begin{array}{l}2.954 \\
2.710 \\
2.687 \\
2.649 \\
2.652 \\
2.662 \\
2.640\end{array}$ & $\begin{array}{r}1.45 \\
1.77 \\
.59 \\
.94 \\
.91 \\
1.24 \\
.91\end{array}$ & $\begin{array}{l}181 \\
166 \\
166 \\
164 \\
164 \\
164 \\
163\end{array}$ \\
\hline
\end{tabular}

\section{MINNESOTA}

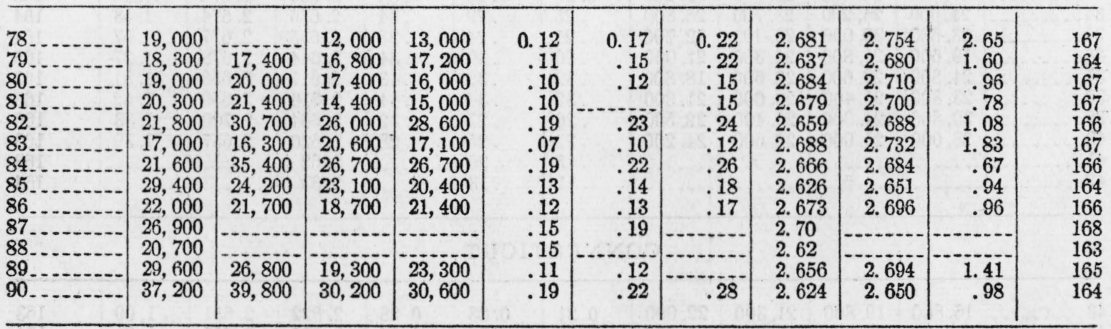


TABLE 3.-Average values obtained for compressive strength, absorption, density, porosity and weight per cubic foot of various granites, by States-Continued

\begin{tabular}{|c|c|c|c|c|c|c|c|c|c|c|c|}
\hline \multirow{3}{*}{ Serial No. } & \multicolumn{4}{|c|}{$\begin{array}{l}\text { Compressive strength perpen- } \\
\text { dicular }(A) \text { and parallel }(B) \text { to } \\
\text { the rift }\end{array}$} & \multicolumn{3}{|c|}{$\begin{array}{l}\text { Absorption by weight } \\
\text { for immersion periods } \\
\text { of }\end{array}$} & \multicolumn{2}{|c|}{ Density } & \multirow{3}{*}{$\begin{array}{l}\text { Poros- } \\
\text { ity a }\end{array}$} & \multirow{3}{*}{$\begin{array}{l}\text { Com- } \\
\text { puted } \\
\text { weight } \\
\text { per } \\
\text { cu. ft. }\end{array}$} \\
\hline & \multicolumn{2}{|c|}{ Specime dry } & \multicolumn{2}{|c|}{ Specimens wet } & \multirow{2}{*}{$48 \mathrm{hr}$} & \multirow{2}{*}{14 days } & \multirow{2}{*}{$1 \mathrm{yr}$} & \multirow{2}{*}{ Bulk } & \multirow{2}{*}{ True } & & \\
\hline & $A$ & $B$ & $A$ & $B$ & & & & & & & \\
\hline
\end{tabular}

\section{MISCELLANEOUS}

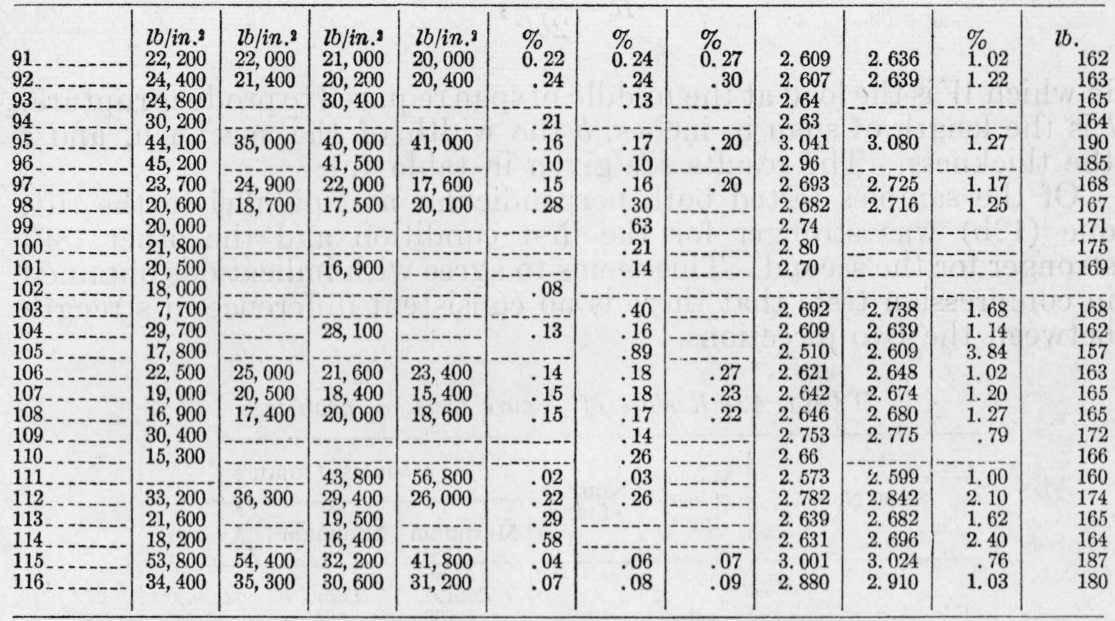

- Total pore space including that of closed pores.

One feature worthy of note regarding some of the granites is the rather large variation in strength shown by specimens cut from a single sample. The deviations of the individual values from the average of each pair of like specimens similarly tested averaged 7 percent for serial numbers 1 to 58 , but the deviation of three of these was more than 20 percent.

The following values, showing the range in compressive strength recorded for other masonry materials, are given for comparison:

\begin{tabular}{|c|c|}
\hline Material & Compressive strength \\
\hline 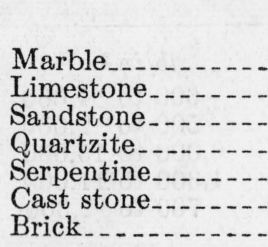 & $\begin{array}{r}l b / \text { in. }^{2} \\
8,000 \text { to } 27,000 \\
2,600 \text { to } 28,000 \\
5,000 \text { to } 20,000 \\
16,000 \text { to } 45,000 \\
11,000 \text { to } 28,000 \\
1,600 \text { to } 21,800 \\
1,000 \text { to } 20,000\end{array}$ \\
\hline
\end{tabular}




\section{FLEXURAL STRENGTH}

Stone in modern construction is seldom subjected to high bending stresses, and hence no systematic study of flexural strength was made on the granites. The modulus of rupture was determined on 29 specimens representing 5 samples. The specimens ( 4 by 12 by 1 in.) were cut, as shown in figure 1, for tests in various directions, supported on adjustable knife edges $10 \mathrm{in}$. apart, and loaded at the center. The modulus of rupture was computed from the formula

$$
R=\frac{3 W l}{2 b t^{2}},
$$

in which $W$ is the load at the middle of span required to produce rupture, $l$ is the length of span in inches, $b$ the width of the specimen, and $t$ the thickness. The results are given in table 4 .

Of the samples tested both perpendicular and parallel to the rift, one $(12 \mathrm{~b})$ was stronger for the first condition and the other (89) stronger for the second. This seems to agree with indications obtained in compression tests that there is no consistent difference in strength between the two directions.

TABLE 4.-Results of flexure tests on granite

\begin{tabular}{|c|c|c|c|c|c|}
\hline \multirow{2}{*}{ Serial No. ${ }^{a}$} & \multirow{2}{*}{$\begin{array}{l}\text { Manner } \\
\text { of load- } \\
\text { ing } b\end{array}$} & \multirow{2}{*}{$\begin{array}{l}\text { Number } \\
\text { of tests }\end{array}$} & \multicolumn{3}{|c|}{ Modulus of rupture } \\
\hline & & & Maximum & Minimum & Average \\
\hline $\begin{array}{l}12 \mathrm{~b} \\
12 \mathrm{~b} \\
12 \mathrm{~b}\end{array}$ & $\begin{array}{l}A \\
B \\
C\end{array}$ & $\begin{array}{l}5 \\
5 \\
5\end{array}$ & $\begin{array}{r}l b / \text { in . }{ }^{2} \\
1,630 \\
1,520 \\
1,560\end{array}$ & $\begin{array}{l}l b / \text { in. }^{2} \\
1,450 \\
1,380 \\
1,410\end{array}$ & $\begin{array}{l}\text { lb/in. }{ }^{2} \\
1,540 \\
1,430 \\
1,490\end{array}$ \\
\hline 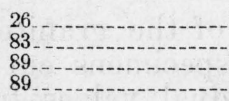 & $\begin{array}{l}A \\
C \\
A \\
B\end{array}$ & $\begin{array}{l}3 \\
2 \\
3 \\
3\end{array}$ & $\begin{array}{l}1,980 \\
3,170 \\
2,450 \\
2,520\end{array}$ & $\begin{array}{l}1,910 \\
2,940 \\
2,180 \\
2,450\end{array}$ & $\begin{array}{l}1,930 \\
3,060 \\
2,290 \\
2,500\end{array}$ \\
\hline $109 \ldots$ & $A$ & 3 & 5,550 & 4,970 & 5,190 \\
\hline
\end{tabular}

a Serial No. $12 \mathrm{~b}$ was from the same quarry as No. 12

- For test $A$ the specimens were prepared as shown in figure 1, at 3; for test $B$ as at 5 ; and for test $C$ as at 4 .

The following values show the range in modulus of rupture recorded for other types of stone:

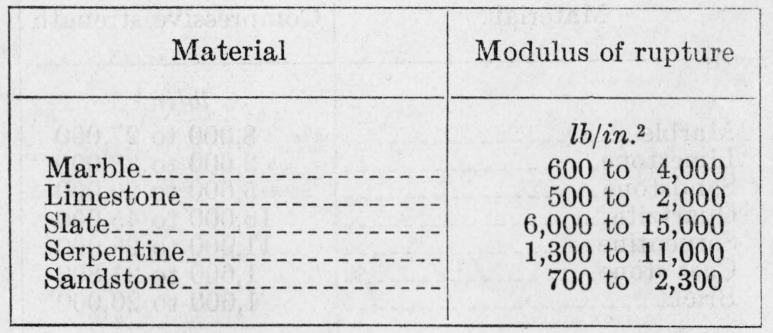




\section{SHEARING STRENGTH}

Shearing strength of granite may be of more concern to the designer of granite structures than the compressive strength, because the latter is usually far in excess of that required for all ordinary conditions. The load on large blocks of granite in masonry is sometimes concentrated near the edge because of improper bedding with mortar, and spalls occur near the edges which may be due in part to high shearing stresses.

A few determinations of shearing strength on granite were made with the punching shear device shown in figure 2, and the results are given in table 5. This device consists essentially of a base plate carrying two vertical guide rods and a 2 -in. plunger provided with a spherical adjustment block at the bottom and a loading table at the top. The function of the coil spring is merely to support the loading table and plunger at a convenient distance above the base plate while the specimen is placed in position. In making the tests, the load necessary to compress the spring until the plunger makes contact with the specimen is determined and subtracted from the load required to punch out a 2 -in. disk.

Specimens on which these tests were made were slabs approximately 4 by 6 by 1 in. The shearing strength was computed by means of the formula

$$
S=\frac{P}{2 \pi t},
$$

in which $P$ is the total load minus the load required to compress the spring and $t$ is the thickness of specimen.

The results given in table 5 range from 3,900 to $4,600 \mathrm{lb} / \mathrm{in}^{2}{ }^{2}$ The ratios between the shearing strength and the corresponding compressive strength of the samples are given in the last column.

TABLE 5.-Results of shear tests on granite

\begin{tabular}{|c|c|c|c|c|c|}
\hline \multirow{2}{*}{ Serial No. ${ }^{\circ}$} & \multirow{2}{*}{$\begin{array}{l}\text { Number } \\
\text { of tests }\end{array}$} & \multicolumn{3}{|c|}{ Shearing strength } & \multirow{2}{*}{ Ratio $S / C^{\circ}$} \\
\hline & & Maximum & Minimum & Average & \\
\hline $\begin{array}{l}12 \mathrm{~b} \\
29 \mathrm{a} \\
83 \\
89\end{array}$ & $\begin{array}{l}2 \\
2 \\
3 \\
3\end{array}$ & $\begin{array}{r}l b / \text { in } .^{2} \\
4,800 \\
4,100 \\
4,700 \\
4,600\end{array}$ & $\begin{array}{r}\text { lb/in. }{ }^{2} \\
4,400 \\
3,700 \\
3,900 \\
4,200\end{array}$ & $\begin{array}{r}\text { lb/in. } .^{2} \\
4,600 \\
3,900 \\
4,600 \\
4,300\end{array}$ & $\begin{array}{r}0.23 \\
.29 \\
.27 \\
.15\end{array}$ \\
\hline
\end{tabular}

- Serial Nos. 12b and 29a were, respectively, from the same quarries as Nos. 12 and 29.

$b$ Shearing strength divided by the compressive strength.

The following results show the range in shearing strength recorded in the literature for other types of stone:

\begin{tabular}{|c|c|}
\hline Material & Shearing strength \\
\hline $\begin{array}{l}\text { Marble } \\
\text { Slate } \\
\text { Limestone } \\
\text { Sandstone }\end{array}$ & $\begin{array}{c}l b / \text { in } .^{2} \\
1,300 \text { to } 6,500 \\
2,000 \text { to } 3,600 \\
800 \text { to } 3,600 \\
300 \text { to } 3,000\end{array}$ \\
\hline
\end{tabular}




\section{ABRASIVE HARDNESS}

The abrasive resistance of granite is of interest when the stone is to be exposed to severe conditions of wear, as in pavements. Three methods ${ }^{2}$ for determining the "hardness" of stone have been used rather extensively in this country, and the results are expressed so differently that the values obtained by one method cannot be converted, by computation, to those of another. Each method has certain merits in a particular field of testing, but some confusion may arise if the particular test used is not properly designated.

The results given in table 6 were obtained by the method developed at the National Bureau of Standards. This method employs a specimen 2 by 2 by 1 in., and usually three such pieces are tested for each sample. They are held on a revolving cast iron disk under a constant pressure of $2 \mathrm{~kg}(4.4 \mathrm{lb})$ and made to revolve at half the speed of the grinding disk. The abrasive is artificial corundum No. 60 , which is fed to the disk at a constant rate. Since the abrasive is much harder than quartz, the wear is more rapid than in the Dorry test. The amount of wear is determined by weighing the specimens before and after the test, and the results are computed by the formula

$$
H_{a}=\frac{10\left(W_{s}+2,000\right) G,}{2,000 W_{a}}
$$

in which $W_{s}$ is the original weight of a specimen, $G$ its bulk density, and $W_{a}$ the loss of weight (all weights in grams).

Comparisons of results obtained with the Dorry and National

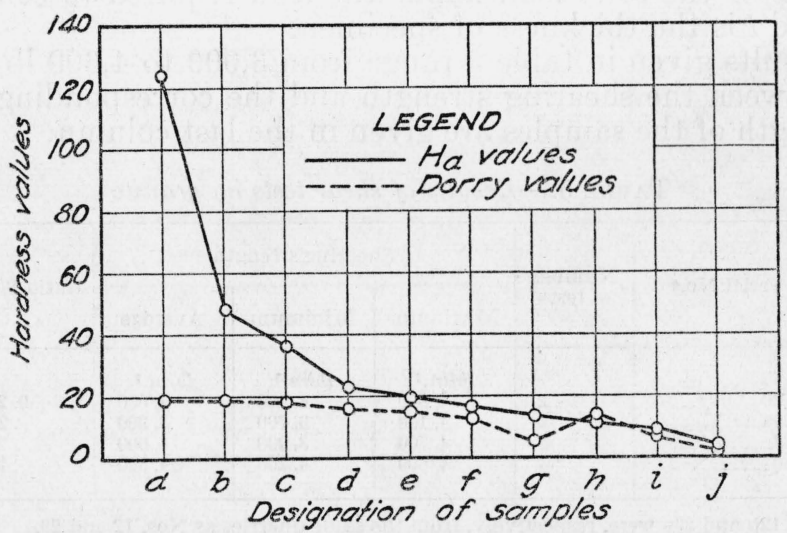

FigURE 3.-Abrasive hardness results on 10 materials, obtained with the Kessler and Dorry tests.

$a=$ serpentine; $b=$ granite; $c, e$, and $g=$ marble; $e, f, i$, and $j=$ limestone; $h=$ slate.

Bureau of Standards abrasion machines, on materials ranging in hardness from soft limestones to very hard serpentine, are shown in figure 3. For soft materials the results are of approximately the same magnitude, but for materials of intermediate hardness the $H_{a}$ values

\footnotetext{
2 The Dorry and the Deval tests are described in Office of Public Roads, Bull. 44, U. S. Dept. of Agriculture, Washington, D. C. The third, designed by D. W. Kessler, is described in detail in BS J. Research 11, 637 (1933) RP612, Wear Resistance of Natural Stone Flooring.
} 


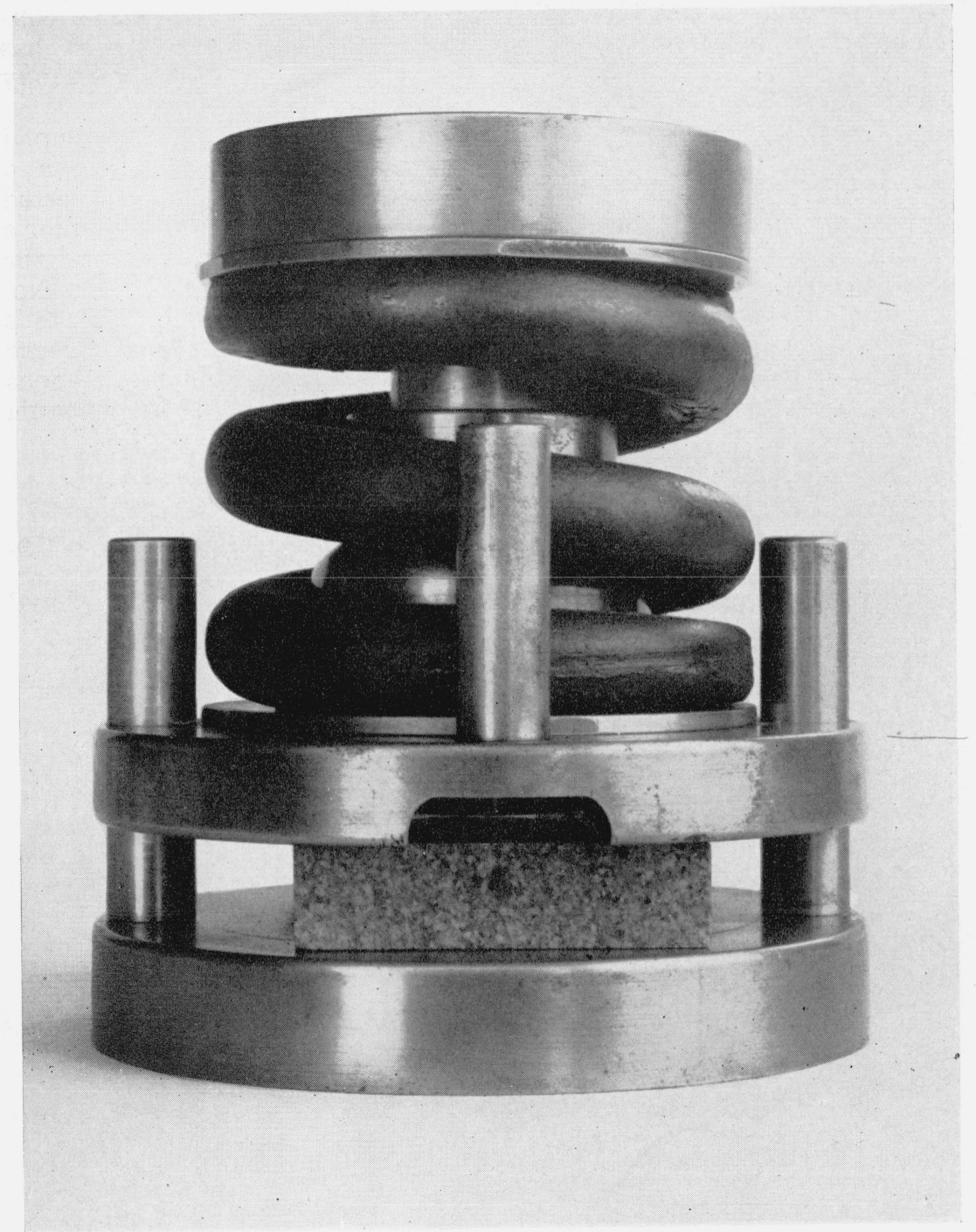

FiguRE 2.-Punching shear testing device with specimen in position for test. 


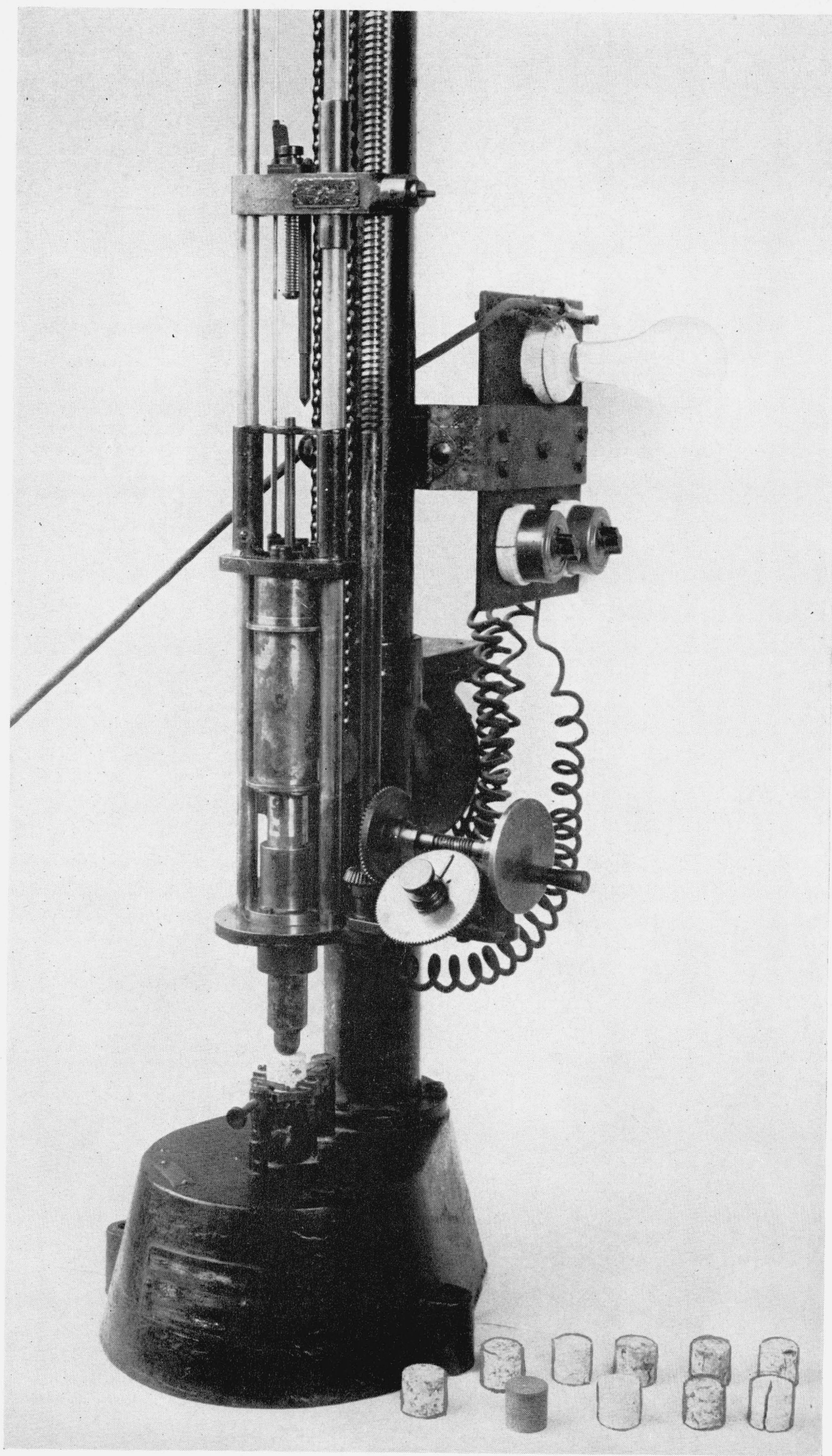

FIGURE 4.-Page impact toughness tester, showing specimens in position and several specimens after test. 
are about 25 percent higher and large differences are shown on hard materials because the Dorry values cannot be greater than 20 .

TABLE 6.-Results of abrasion tests on granite

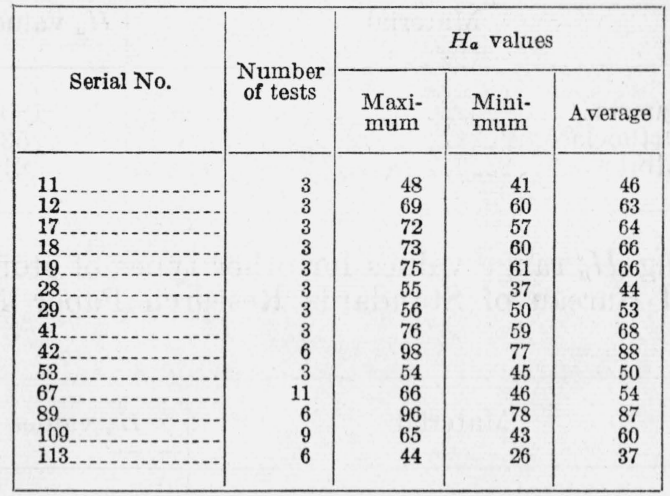

Comparisons of results (see footnote 2) obtained in the Dorry and Deval tests on 40 samples of granite show that the Dorry values are, on the average, about 20 percent higher than the "French coefficients" of the Deval test. Since the Deval test involves the toughness factor, the ratio between the two varies considerably. Materials that have high toughness values usually show a correspondingly high French coefficient.

The results in table 6 on granites from 14 producing districts show a range in $H_{a}$ values of 37 to 88 , indicating that some granites are much more resistant to abrasion than others. In comparison with most other types of stone these values are all high, and good resistance to wear may be expected from any sound granite in the floors or steps of buildings. Probably the greatest concern regarding granite in such applications should be whether it will wear to a smooth surface and become slippery.

In some of the tests which were repeated on the same specimens, it was noted that the second test gave higher $H_{a}$ values than the first. This seems to indicate that granite is injured to a slight depth in finishing. Surfacing methods which employ a bush hammer or pneumatic tool are rather severe, and they probably shatter the more brittle minerals to some extent. Specimens of a granite which is used extensively in building construction were tested three times on one face, and the results were as follows:

\begin{tabular}{|c|c|}
\hline Test & $H_{a}$ values \\
\hline First (on original surface) & \\
Second _... & 41 \\
Third_...... & 49 \\
\hline
\end{tabular}

The total depth ground off from each specimen was about 0.01 in., and the indicated increase in hardness was nearly 27 percent. 
A few hardness tests have been made on the principal minerals of granite which indicate that quartz is more than twice as resistant to wear as the feldspars. The results were as follows:

\begin{tabular}{|c|c|}
\hline Material & $H_{a}$ values \\
\hline $\begin{array}{l}\text { Quartz } \\
\text { Orthoclase_... } \\
\text { Albite...... }\end{array}$ & $\begin{array}{r}180 \\
53 \\
81\end{array}$ \\
\hline
\end{tabular}

The following $H_{a}$ range values for other types of stone are quoted from National Bureau of Standards Research Paper No. 612 (see footnote 2):

\begin{tabular}{|c|c|}
\hline Material & $H_{a}$ values \\
\hline $\begin{array}{l}\text { Marble } \\
\text { Limestone } \\
\text { Sandstone } \\
\text { Slate } \\
\text { Serpentine. } \\
\text { Travertine. }\end{array}$ & $\begin{array}{r}8 \text { to } 42 \\
1 \text { to } 24 \\
2 \text { to } 26 \\
6 \text { to } 12 \\
13 \text { to } 110 \\
1 \text { to } 16\end{array}$ \\
\hline
\end{tabular}

\section{TOUGHNESS}

The resistance of a stone to impact is a measure of its tenacity, and this property is commonly called "toughness." Stones of low toughness are apt to spall when sharp corners are exposed to rough usage, such as occurs on steps and on some other members near the base of buildings.

The samples for these tests were selected with the idea of showing the range in toughness values, and the tests were made on a Page impact apparatus (fig. 4). In this apparatus the specimen is mounted securely on a heavy cast-iron base. A steel plunger with the lower end rounded rests on the specimen, and a $2-\mathrm{kg}$ weight is dropped on the plunger by a motor-driven sprocket chain. The height of the first drop is $1 \mathrm{~cm}$ and each succeeding drop is increased by $1 \mathrm{~cm}$. Since the force of the impact is concentrated on the center of the test specimen by the hemispherical contact of the plunger, the specimen usually breaks in three or four approximately equal segments.

This test has been used extensively in selecting road, paving, and curb materials. Results of a large number of tests on various types of stone have been published by the United States Bureau of Public Roads. ${ }^{3}$

Table 7 gives data on 10 samples tested perpendicular to the rift, 2 of which were also tested parallel to the rift. Very little difference in toughness is indicated for the two directions. The average values

\footnotetext{
3 Albert T. Goldbeck and Frank H. Jackson, Jr., The Physical Testing of Rock for Road Building, U. S. Dept. of Agr., Office of Public Roads, Bul. No. 44 (1912).

Prevost Hubbard and Frank H. Jackson, Jr., Results of Physical Tests of Road-Building Rock, U. S. Dept. of Agr., Bul. No. 370 (1916).

The Results of Physical Tests of Road-Building Rock from 1916 to 1921, Inclusive, U. S. Dept. of Agr., Bul. No. $1132(1923)$.
} 
ranged from 8 to 27 , the highest being obtained on a comparatively fine-grained granodiorite.

TABLE 7.-Results of toughness tests on granite

\begin{tabular}{|c|c|c|c|c|c|}
\hline \multirow{2}{*}{ Serial No. } & \multirow{2}{*}{$\begin{array}{l}\text { Direction } \\
\text { of } \\
\text { impact a }\end{array}$} & \multirow{2}{*}{$\begin{array}{c}\text { Number } \\
\text { of } \\
\text { tests }\end{array}$} & \multicolumn{3}{|c|}{ Toughness values } \\
\hline & & & $\begin{array}{l}\text { Maxi- } \\
\text { mum }\end{array}$ & $\begin{array}{l}\text { Mini- } \\
\text { mum }\end{array}$ & A verage \\
\hline $\begin{array}{r}13 \\
21 \\
21 \\
55 \\
63 \\
89 \\
89 \\
106\end{array}$ & $\begin{array}{l}A \\
A \\
B \\
A \\
A \\
A \\
A \\
A \\
B \\
A \\
A \\
A\end{array}$ & $\begin{array}{l}3 \\
3 \\
3 \\
3 \\
3 \\
5 \\
3 \\
3 \\
3 \\
3 \\
3 \\
3\end{array}$ & $\begin{array}{r}11 \\
11 \\
9 \\
13 \\
25 \\
16 \\
8 \\
11 \\
10 \\
10 \\
28 \\
21\end{array}$ & $\begin{array}{r}7 \\
9 \\
8 \\
12 \\
19 \\
11 \\
8 \\
8 \\
8 \\
7 \\
26 \\
16\end{array}$ & $\begin{array}{r}10 \\
9 \\
8 \\
13 \\
22 \\
13 \\
8 \\
9 \\
9 \\
9 \\
27 \\
19\end{array}$ \\
\hline
\end{tabular}

a $A$ signifies that the direction of impact was perpendicular to the rift and $B$ parallel to the rift.

The following range and average for toughness results on other types of stone have been taken from publications of the United States Bureau of Public Roads (see footnote 3 ):

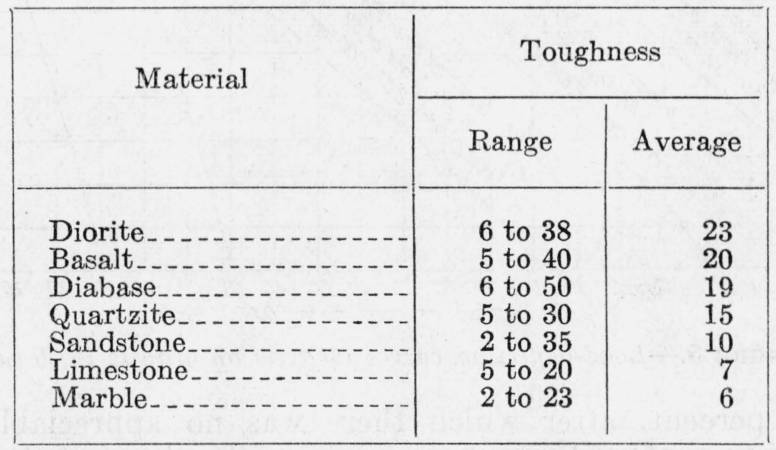

\section{ELASTICITY}

The elasticity of granite in flexure was determined on serial Nos. 12 and 109. The specimens were 4 by 12 by 1 in., and they were supported on knife-edges of the rocker type $10 \mathrm{in}$. apart, loaded at midspan. The values were calculated by the formula

$$
E=\frac{W l^{3}}{4 \Delta b t^{3}},
$$

in which $W$ is the load in pounds, $l$ is the length of span, $\Delta$ is the deflection at midspan for load $W, b$ is the width, and $t$ is the thickness of specimen. Deflection readings were made by means of a deflectometer suspended from four points on the neutral axis of the specimen above the knife-edges. 
In these tests 15 specimens of serial No. 12 were used, 5 being prepared in each of 3 directions with respect to the rift, as shown in figure 1. The load-deflection curves are given in figure 5 for one typical test of each group. These curves show that the Hooke's law does not apply for this granite except within a small range of loading. The modulus of elasticity values shown in figure 5 for each test were computed from points on the curve where the stress-strain ratio was nearly constant. The values for serial No. 12 are much lower than any of the published results for elasticity of granite in compression.

One specimen of granite No. 12 was held under a constant load of $100 \mathrm{lb}$ for 10 days. During the first 6 days the deflection increased

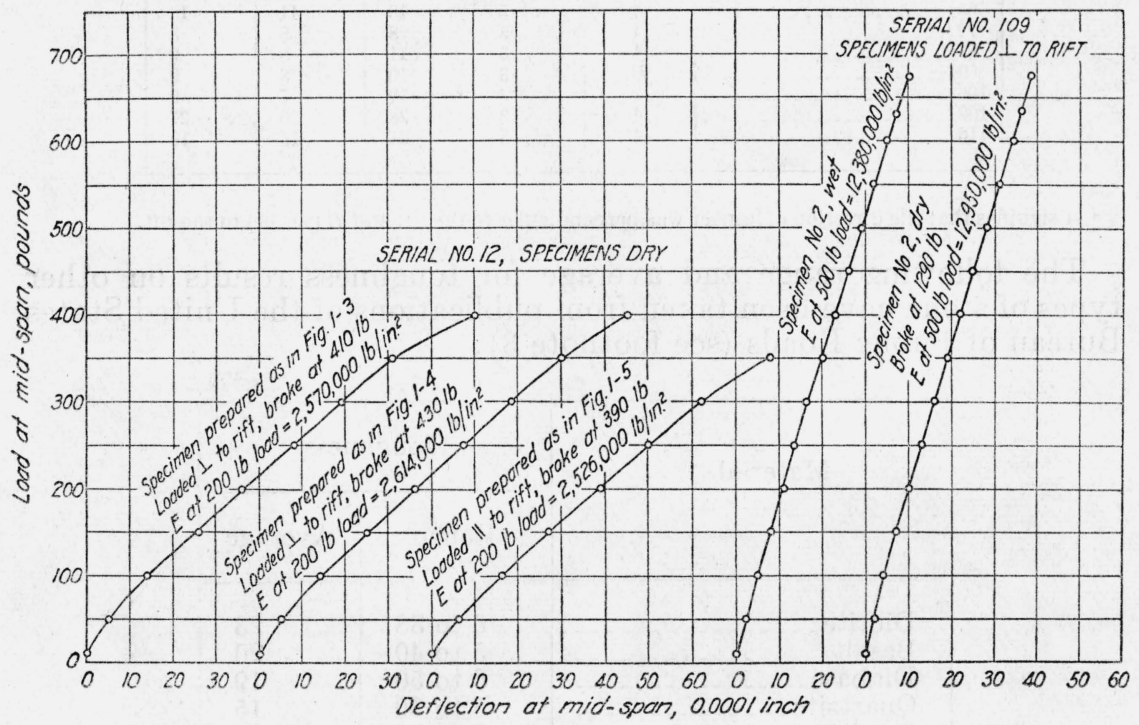

Figure 5.-Load-deflection curves for tests on granite in flexure.

about 26 percent, after which there was no appreciable change. This seems to confirm the indications from the shape of the curves in figure 5 that such a granite would warp if subjected to flexural stress for a considerable period of time.

Six specimens of serial No. 109 were prepared, three being tested dry and three wet (after soaking 2 days in water). A typical curve for each group is given in figure 5 . The results differ widely from those obtained on serial No. 12 in that the proportionality was constant within a considerable range of loading and the modulus of elasticity values were much higher.

Modulus of elasticity determinations made at Watertown Arsenal ${ }^{4}$ on five granites show that the ratio of stress to strain in compression increases with increasing loads. The specimens were 4 by 6 by 24 in. and the gage length was 20 in. The stress-strain curves in figure 6 have been reproduced from data obtained in these tests. The results

4 Report of the Test of Metals and Other Materials for Industrial Purposes at Watertown Arsenal, Mass., Ordnance Dept., U. 8. Army, p. 393-401 (1894). 
are of considerable interest on account of the unusually large specimens and the large number of strain readings, and therefore some of the data are quoted in table 8.

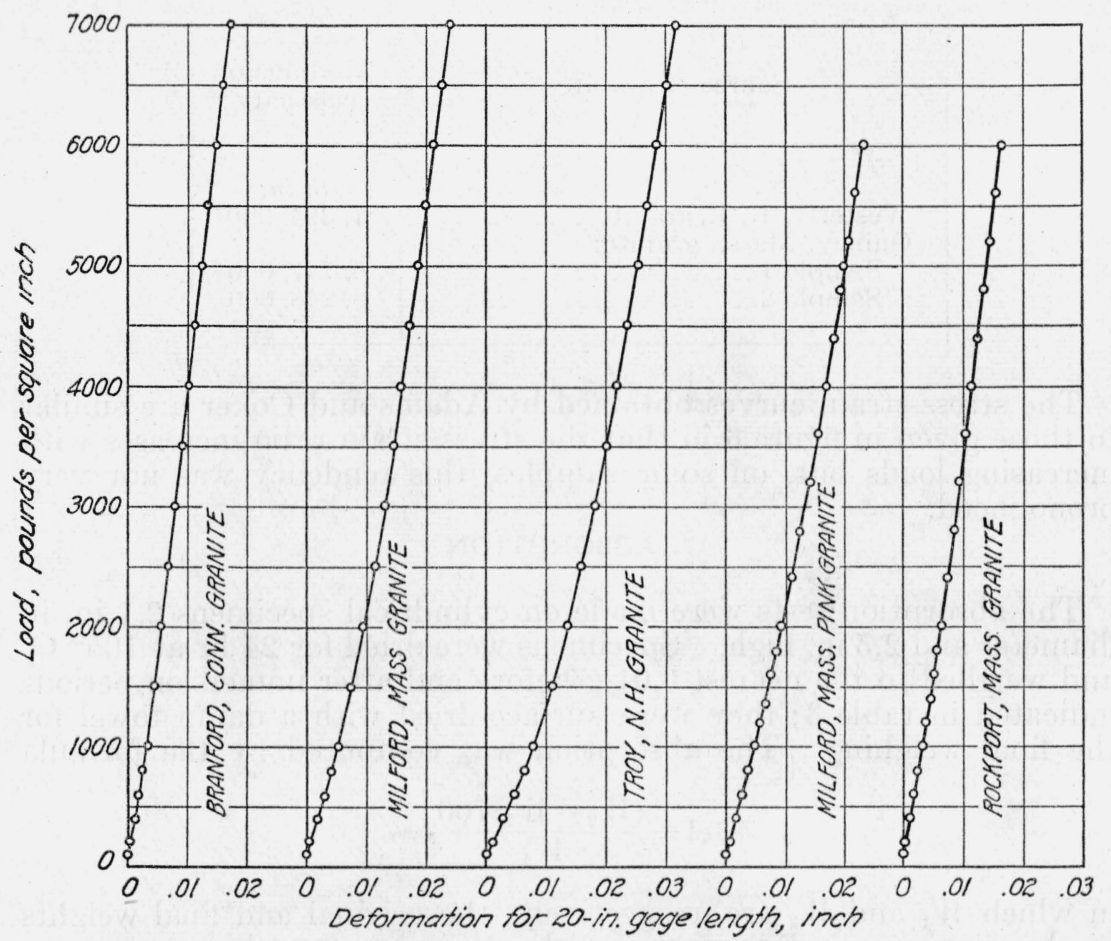

Figure 6.-Load-duformaiion curves for tests on granite in compression.

Reproduced from tests at Watertown Arsenal; Report of the Test of Metals and other Materials for Industrial Purposes at Watertown Arsenal, Mass., Ordnance Dept., U. S. Army, p. 393-401 (1894).

TABLE 8.-Modulus of elasticity $(E)$ results on five granites.

[Tests made at Watertown Arsenal, Report of the Test of Metals and Other Materials for Industrial Purposes at Watertown Arsenal, Mass., Ordnance Dept., U. S. Army, p. 393-401 (1894) ]

\begin{tabular}{|c|c|c|}
\hline Source of granite & $\begin{array}{l}\text { Load range for } \\
\text { which } E \text { values } \\
\text { were computed }\end{array}$ & $\begin{array}{l}\text { Modulus of } \\
\text { elasticity in } \\
\text { compression } \\
(E)\end{array}$ \\
\hline $\begin{array}{l}\text { Branford, Conn } \\
\text { Milford, Mass. (pink granite) } \\
\text { Troy, N. H } \\
\text { Milford, Mass.- } \\
\text { Rockport, Mass }\end{array}$ & $\begin{array}{c}\text { lb/in. } .^{2} \\
1,000 \text { to } 3,000 \\
1,000 \text { to } 2,000 \\
1,000 \text { to } 3,000 \\
1,000 \text { to } 3,000 \\
1,000 \text { to } 2,000\end{array}$ & $\begin{array}{l}\text { lb/in.2 } \\
8,333,300 \\
5,128,000 \\
4,545,400 \\
5,633,800 \\
6,666,700\end{array}$ \\
\hline
\end{tabular}

Adams and Coker ${ }^{5}$ determined the modulus of elasticity in compression of seven samples of granite, using prisms 1 in. square in cross section and 3 in. long, or, in some cases, cylinders 1 in. in diameter and

\footnotetext{
S Frank D. Adams and Ernest G. Coker, An Investigation Into the Elastic Constants of Rock, More Especially with Reference to Cubic Compressibility, published by the Carnegie Institute of Washington, D. C. (June 1906).
} 
3 in. long. Their tests gave modulus of elasticity values ranging from 5,685,000 to 8,295,000 lb/in. ${ }^{2}$ The series included three domestic granites for which the elasticity values are:

\begin{tabular}{|c|c|}
\hline Source of granite & $\begin{array}{l}\text { Modulus of } \\
\text { elasticity }\end{array}$ \\
\hline $\begin{array}{l}\text { Westerly, R. I., granite } \\
\text { Quincy, Mass., granite: } \\
\text { Sample 1. } \\
\text { Sample 2... }\end{array}$ & $\begin{array}{c}l b / \text { in } \\
7,394,000 \\
6,747,000 \\
8,248,000\end{array}$ \\
\hline
\end{tabular}

The stress-strain curves obtained by Adams and Coker are similar to those given in figure 6 in that the stress-strain ratio increases with increasing loads but, on some samples, this tendency was not very pronounced.

\section{ABSORPTION}

The absorption tests were made on cylindrical specimens $2.1 \mathrm{in}$. in diameter and $2.5 \mathrm{in}$. high. Specimens were dried for $24 \mathrm{hr}$ at $105^{\circ} \mathrm{C}$, and weighed to the nearest $0.01 \mathrm{~g}$ before and after immersion periods indicated in table 3 ; they were surface-dried with a damp towel for the final weighing. The absorption was computed by the formula

$$
A=\frac{\left(W_{2}-W_{1}\right) 100}{W_{1}},
$$

in which $W_{1}$ and $W_{2}$ are, respectively, the original and final weights of the specimens. For most samples the average values stated for the 48-hr and 14-day periods in table 3 represent eight individual tests, whereas the 1-year tests were made on four specimens.

The results indicate that there are small increases in absorption for all samples with the time of immersion up to 1 year. A few samples absorbed approximately 100 percent more water at the end of 1 year than they had absorbed during $48 \mathrm{hr}$, but for most samples the increases at the end of 1 year were less than 50 percent of the $48-\mathrm{hr}$ values. In general, the normal granites from the midwestern States showed considerably lower absorption than those of the eastern States, and the dark-colored materials (gabbros and basalts) usually showed very low absorption. The average absorption of all samples in these tests for 2 days' immersion was 0.24 percent and for 1 year 0.28 percent. The high values obtained for a few samples $(99,103$, and 105) which were known to have been taken from near the surface of the ground seemed to indicate that the absorption test may be of value in checking the quality of shipments from any given quarry. 
The following ranges in values for certain other types of masonry materials are given for comparison:

\begin{tabular}{|c|c|}
\hline Material & $\begin{array}{c}\text { Absorption, by } \\
\text { weight }\end{array}$ \\
\hline $\begin{array}{l}\text { Marble a } \\
\text { Slate- } \\
\text { Quartzite- } \\
\text { Sandstone- } \\
\text { Cast stone- } \\
\text { Brick }\end{array}$ & $\begin{array}{l}\% \\
0.06 \text { to } 0.45 \\
0.01 \text { to } 0.60 \\
0.1 \text { to } 2.0 \\
2.0 \text { to } 12.0 \\
2.5 \text { to } 18.2 \\
0.2 \text { to } 30.0\end{array}$ \\
\hline
\end{tabular}

a This range is based on tests of the usual types of domestic marble. Tests on some of the highly colored brecciated foreign marbles have given results much higher than 0.45 percent.

\section{DENSITY}

(a) BULK DENSITY

Bulk-density values $\left(D_{b}\right)$ given in table 3 were obtained in conjunction with the absorption test. These specimens weighed approximately $400 \mathrm{~g}$, and all weights were determined to the nearest $0.01 \mathrm{~g}$. They were weighed suspended in water after soaking for $48 \mathrm{hr}$. The results were computed by the formula

$$
D_{b}=\frac{W_{1} d}{W_{2}-W_{3}},
$$

in which $W_{1}$ is the dry weight, $W_{2}$ the weight in air after soaking 48 $\mathrm{hr}, W_{3}$ the weight of soaked specimens suspended in water, and $d$ the density of the water in which the suspended weight was obtained.

The bulk-density values given in table 3 are, for most samples, based on tests of eight specimens. For the entire series of samples, the values range from 2.60 to 3.04 , the high value being for a "black granite" (gabbro). Results for the normal granites are mostly between 2.6 and 2.7 .

\section{(b) TRUE DENSITY}

Density determinations were made on 55 -g powdered samples by determining the volumes with Le Chatelier flasks. About $300 \mathrm{~g}$ of fragments were selected from specimens after having been crushed in the compression test. These were ground in a mortar until all of the sample passed a No. 100 sieve. The powdered material was dried at $105^{\circ} \mathrm{C}$; and after being cooled in a desiccator, it was stored in sealed glass jars until tested. Alcohol was used for the immersion liquid. This method was checked for accuracy against picnometer methods, and it was found that by carefully controlling the temperature of the liquid in the Le Chatelier flasks it gave somewhat closer repeat values on the same sample than the picnometer.

The results are given in table 3 . The highest value was 3.080 for a gabbro and the lowest 2.599 for a normal biotite granite. 
(c) WEIGHT PER CUBIC FOOT

The weights per cubic foot in table 3 were computed by multiplying the bulk density by 62.35 . The following ranges in weight per cubic foot for other types of material are given for comparison:

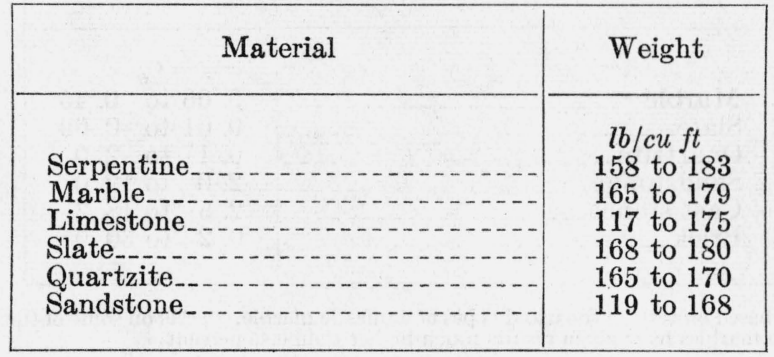

\section{POROSITY}

Porosity values were computed from the bulk-density and truedensity values by the formula

$$
P=\frac{\left(D_{a}-D_{b}\right) 100}{D_{a}},
$$

in which $D_{a}$ and $D_{b}$ are, respectively, the true density and bulk density. This method gives the total pore space regardless of enclosed pores. Microscopic studies indicate that frequently the quartz and sometimes other constituents of granite contain minute cavities which have no outlets. The porosity values obtained on various granites are given in table 3 . The average of the series was 1.29 percent and the range 0.40 to 3.84 percent.

The degree of saturation in the various absorption tests may be computed by the formula

$$
s=\frac{A D_{b}}{d P} .
$$

For ordinary purposes the value of $d$ may be considered as unity. Computations of the degree of saturation for 45 samples of granite from eastern States for the 1-year absorption tests gave an average of 0.66 . Similar computations for 17 samples from midwestern States gave an average of 0.44 .

The following range values for porosity of other masonry materials

\begin{tabular}{|c|c|}
\hline Material & Porosity \\
\hline $\begin{array}{l}\text { Marble } \\
\text { Slate } \\
\text { Quartzite- } \\
\text { Sandstone-2 } \\
\text { Limestone- } \\
\text { Cast stone }\end{array}$ & $\begin{array}{l}\quad \% \\
0.4 \text { to } 2.1 \\
0.1 \text { to } 1.7 \\
1.5 \text { to } 2.9 \\
1.9 \text { to } 27.3 \\
1.1 \text { to } 31.0 \\
6.7 \text { to } 32.7\end{array}$ \\
\hline
\end{tabular}
are given for comparison: 


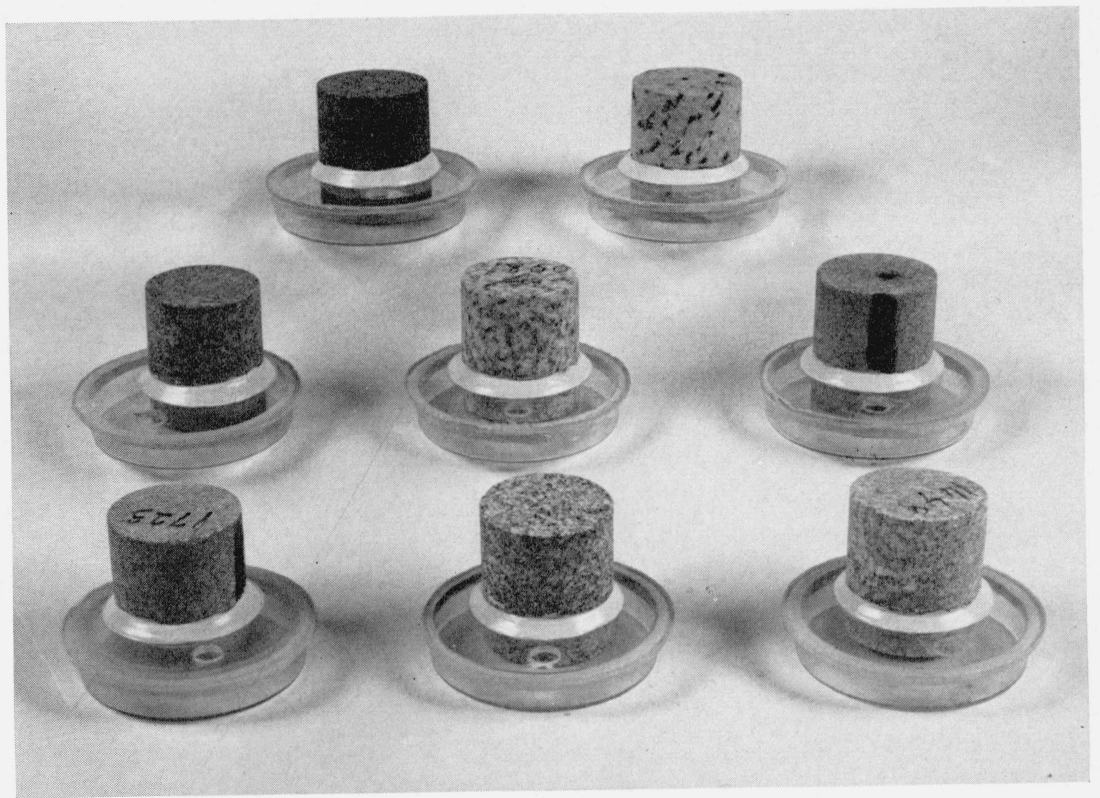

Figure 7.-Water-transmission tests. 


\section{WATER TRANSMISSION RATE}

Granite is used frequently in the base courses of buildings in which the superstructure is of a more porous material. To a large extent the granite courses prevent the rise of moisture by capillarity from the ground. Differences in porosity or absorption of different granites indicate that some may be better adapted to grade-course construction than others. Hence a test was devised to compare the rates at which different samples allow water to pass through the pores by capillarity.

The tests were made by sealing specimens $2.1 \mathrm{in}$. in diameter by 2.5 in. high in shallow glass vessels containing a small amount of distilled water (fig. 7), and determining the rates of loss in weight at suitable intervals. In this test no attempt was made to control the tempera-

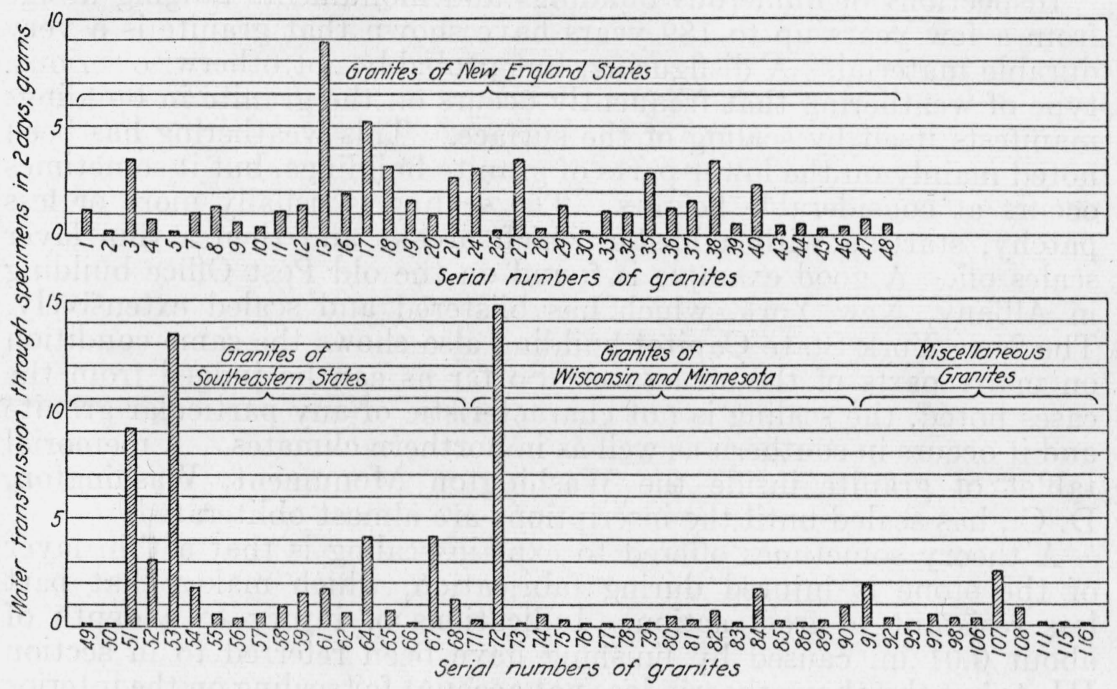

FiguRe 8.-Results of water-transmission tests on 85 granites.

ture or hurnidity, because moisture passed through the pores so slowly that the surface above the cover plate remained dry. Several tests were made by placing an impervious membrane around the vertical side of the specimen so evaporation could occur only at the top. When this was done, a period of several days was required for the weight losses to reach a constant value and the time was variable for different granites. Without the membrane the rate of loss became constant for most samples in $48 \mathrm{hr}$, and this period was used for determining the comparative rates. Several samples were weighed daily for 15 days, and a slight decrease in the rate of evaporation occurred after about 3 days.

The results for 85 samples of granite are shown in figure 8. For more than half of the samples the water transmitted in $48 \mathrm{hr}$ was less than $1 \mathrm{~g}$, the greatest amount transmitted was nearly $15 \mathrm{~g}$, and the average of all was $1.62 \mathrm{~g}$. There appears to be no definite relation between the porosity or absorption values and the transmission rates, except that those granites of very low absorption also have low transmission rates. The transmission rates show a much wider range of 
values than either the absorption or porosity, and this test may give information on the pore structure that is not indicated by absorption or porosity determinations.

The sample that gave the highest transmission rate (No. 72) was one which showed unmistakable signs of alteration from its original state by weathering. This suggests that the transmission test may be a more definite indicator than the absorption test for determining whether different consignments of granite have been quarried near weathered zones.

\section{DURABILITY STUDIES}

\section{OBSERVATIONS ON STRUCTURES}

Inspections of numerous buildings and monuments ranging in age from a few years up to 189 years have shown that granite is a very durable material. A disfiguring, but probably not otherwise serious, type of weathering that frequently occurs on the granite in buildings manifests itself by scaling of the surface. This weathering has been noted mainly on the lower parts of granite buildings, but it sometimes occurs at considerable heights. The scaling is usually more or less patchy, starting with a blister which breaks and then a thin layer scales off. A good example is found on the old Post Office building in Albany, New York, which has blistered and scaled extensively. The New York State Capitol building also shows the same condition on many parts of the structure. So far as can be judged from the cases noted, the scaling is not characteristic of any particular granite and it occurs in southern as well as in northern climates. A memorial tablet of granite inside the Washington Monument, Washington, D. C., has scaled until the inscriptions are almost obliterated.

A theory sometimes offered to explain scaling is that a thin layer of the stone is injured during fabrication, which makes that part less resistant to frost action. Indications of injury to a depth of about 0.01 in. caused by finishing have been referred to in section III-4; but the above theory does not account for scaling on the interior of structures, where neither temperature nor moisture conditions are favorable for frost action. Furthermore, observations indicate that scaling occurs as frequently on modern buildings as on old ones, although modern finishing methods are probably much less severe than those formerly used. The granite for King's Chapel in Boston (built in 1750) was finished by crude and very severe methods, but there is little visible disintegration on this structure.

Blistering and scaling of granite monuments seem to be rare, and only one pronounced example is known to the authors. This is a large monument in Rock Creek Cemetery (Washington, D. C.) which shows scaling over a considerable portion of the surface, and the polished die is so badly scaled that some of the inscriptions are almost illegible.

\section{STUDIES OF GRANITE WEATHERING}

A few samples of granite from old buildings were studied to determine the effects of weathering. Two blocks from a building 100 years old were cored and tested for absorption and compressive strength. One of the samples was identified as being from the same quarry as serial No. 3 in table 1 . The tests showed no loss of strength 
or increase in absorption for the weathered sample. The strength and absorption values of the other weathered sample were near the average of these properties obtained on all samples tested, but no newly quarried sample of the same granite was available for a more definite comparison. The results, therefore, indicate that 100 years of weathering had not changed the physical properties of these granites sufficiently to be measurable.

Chemical and microscopic studies have been made on a few samples of weathered granite, and the results showed that variable amounts of gypsum were present. This indicates that granite scaling may be caused by the crystallization of this salt in the pores of surface layers. The amount of such salt in samples taken from exposed parts of structures evidently depends largely on the time of sampling. In summer, a considerable part of the soluble matter may be carried away by rains, hence samples collected in winter are more indicative. Table 9 gives the results of studies on five samples.

TABLE 9.-Chemical and microscopic studies on weathered granite

\begin{tabular}{|c|c|c|c|c|c|}
\hline Sample & Source and description of samples & $\begin{array}{l}\text { Water- } \\
\text { soluble } a\end{array}$ & Sulfur & $\begin{array}{l}\text { Calcium } \\
\text { sulfate }\end{array}$ & $\begin{array}{c}\text { Calcium } \\
\text { carbonate }\end{array}$ \\
\hline A.- & Surface scales from Albany Post Office, collected in & $\%_{2.2}$ & $\begin{array}{l}\% \\
0.41\end{array}$ & $\begin{array}{l}\% \\
1.72\end{array}$ & $\begin{array}{l}\% \\
0.67\end{array}$ \\
\hline B. & $\begin{array}{l}\text { winter. } \\
\text { From Albany Post Office, } 3 \text { in. back of exposed }\end{array}$ & .5 & .01 & .07 & .82 \\
\hline C. & Surface scales from Cleveland Post Office where & 1.7 & & & \\
\hline & $\begin{array}{l}\text { freely exposed to rains. Collected in summer. } \\
\text { Surface scales from retaining wall of Cleveland }\end{array}$ & 40.0 & & & \\
\hline E.. & $\begin{array}{l}\text { Post Office, not exposed to rains. } \\
\text { Surface scales from Indianapolis Post Office where } \\
\text { exposed to rains. Collected in winter. }\end{array}$ & 2.9 & & & \\
\hline
\end{tabular}

- Amounts were determined gravimetrically and the crystals identified with a petrographic microscope as being mainly gypsum.

It will be seen that the surface samples contained variable amounts of calcium sulfate (probably as gypsum), which substance is not found in newly quarried granites. Sample $B$ shows that calcium sulfate (probably as gypsum) was present in small amounts at a depth of 3 in. from the surface. Sample $D$ from granite masonry not exposed to rains showed a large accumulation of this salt, but the distintegration at that place was no more conspicuous than where sample $C$ was taken. The difference in calcium carbonate for samples $A$ and $B$ may have been due to a natural variation, because sample $A$ was taken from various parts of the buildings.

The calcium sulfate ${ }^{6}$ in weathered granite may have been formed by the action of sulfurous or sulfuric acids on the calcium carbonate originally present in the granite. Sulfur dioxide from the combustion of coal is usually present in the air, and this gas readily forms acids in the presence of moisture. When a particle of calcium carbonate is converted to gypsum, there is an increase in volume and the latter requires a space approximately twice that of the calcium carbonate. The conversion of calcium-carbonate particles in granite to gypsum would, therefore, cause internal stresses, which may be one reason for granite scaling.

\footnotetext{
6 The action of calcium sulfate in the weathering of limestone has been studied by A. Scott Russel, Report of the Building Research Board (1927) Dept. of Sci. and Ind. Res., Westminster, England. The same salt has been found by D. W. Kessler and W. H. Sligh to be a factor in the weathering of slate, Physical Properties and Weathering Characteristics of Slate, BS J. Research 9, 377 (1932) RP477.
} 
The crystal growth of gypsum from sources outside the granite may be a possible cause of granite scaling. About 4 percent by weight of gypsum is used in portland cement, and hence a 1:3 mortar contains approximately 1 percent. A computation will show that 1 volume of such a mortar contains a volume of gypsum almost sufficient to fill the pores of an equal volume of an average granite. If the gypsum is leached from the mortar and concentrated in a surface layer of the granite, it might cause or contribute to scaling. A test, made to determine if granite could be disintegrated by allowing gypsum to crystallize in the pores, gave negative results. In this test several granite specimens were partly immersed in a saturated solution of calcium sulfate for 3 years so that the solution could pass upward through the pores and evaporate on the surfaces exposed to the air. None of the specimens scaled and very little efflorescence occurred.

Experiments made by leaching specimens with a 10-percent solution of sulfuric acid resulted in complete disintegration of one granite within 30 days and a few others within 3 months. Scaling occurred on some of the granites in a manner resembling that found on buildings, but with this relatively strong acid solution the disintegration appeared to be, in part, due to alteration of the biotite. When the disintegrated material was leached with distilled water and the leaching water filtered and evaporated, a considerable amount of gypsum crystals formed in the evaporating dish. This formation of gypsum supports the observation, made in connection with the study of scales from weathered granite, that the conversion of calcite particles in the granite to gypsum is a major cause of scaling. Under actual structural conditions this process may be aided by recrystallization of gypsum from other sources.

\section{S'TUDIES OF WEATHERING AGENTS}

(a) FROST ACTION

Freezing tests were made on 14 samples described in table 1 under serial Nos. 6, 14, 26, 53, 54, 60, 67, 69, 70, 87, 103, 104, 105, and 110. The specimens were frozen 4,500 times under conditions simulating those in masonry walls above the ground level. Before the first freezing the specimens were soaked for two weeks in water, then placed in wire baskets (free to drain) in the freezing chamber at a temperature of about $-12^{\circ} \mathrm{C}$. The freezing period was at least $6 \mathrm{hr}$. Thawing was done by immersing the specimens in water at about $20^{\circ} \mathrm{C}$ for $1 \mathrm{hr}$. After this series of freezings, the test was continued by freezing the specimens 500 times while standing in $1 / 2$ in. of water.

None of these samples showed any visible signs of disintegration after the total 5,000 cycles, and the water-transmission rates of the specimens were no higher than those of specimens of the same granites not subjected to freezing. The samples in this test were probably no more resistant to freezing than any of the others, but the low strengths and high absorption as well as the mineral alterations on some, especially serial Nos. 103 and 105, indicated that they had undergone considerable weathering before the freezing tests were started. These two granites would be considered of inferior quality as judged by their strength and absorption; but since they, as well as all of the others, showed no disintegration in the prolonged freezing test, it seems evident that frost action is of minor importance in the weathering of granite. 

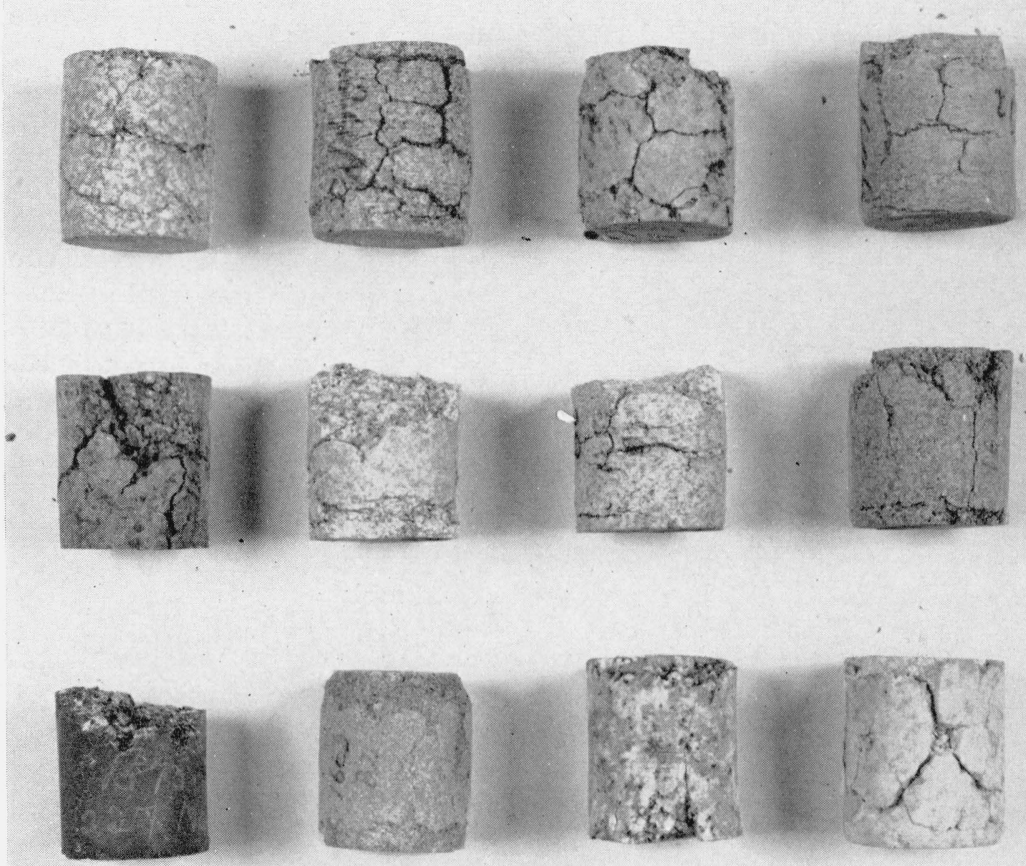

FIGURE 9.-Specimens after sodium-sulfate crystallization tests. 


\section{(b) CRYSTALLIZATION TEST}

The sodium-sulfate crystallization (or soundness) test was applied to 79 samples, and the results are given in table 10 . The procedure consisted in soaking cylindrical specimens 2.1 in. in diameter by 2.5 in. high for $17 \mathrm{hr}$ in a saturated solution of sodium sulfate and drying for $7 \mathrm{hr}$ at $105^{\circ} \mathrm{C}$. This cycle was repeated until an advanced stage of disintegration occurred in the specimens, as shown in figure 9. A similar test is used frequently in selecting aggregate materials for concrete, and the usual requirement is that the samples shall pass five cycles without losing more than 10 percent of the original weight. None of the samples of granite failed in less than 14 cycles and the average was 42 .

This test may give an indication of the relative resistance of different granites to weathering caused by the crystallization of watersoluble salts in the pores as pointed out in section IV -2 . The results obtained agree with those of other investigators in that no correlation with freezing tests was found.

TABLE 10.-Results of crystallization test on various granites (sodium sulfate), by States

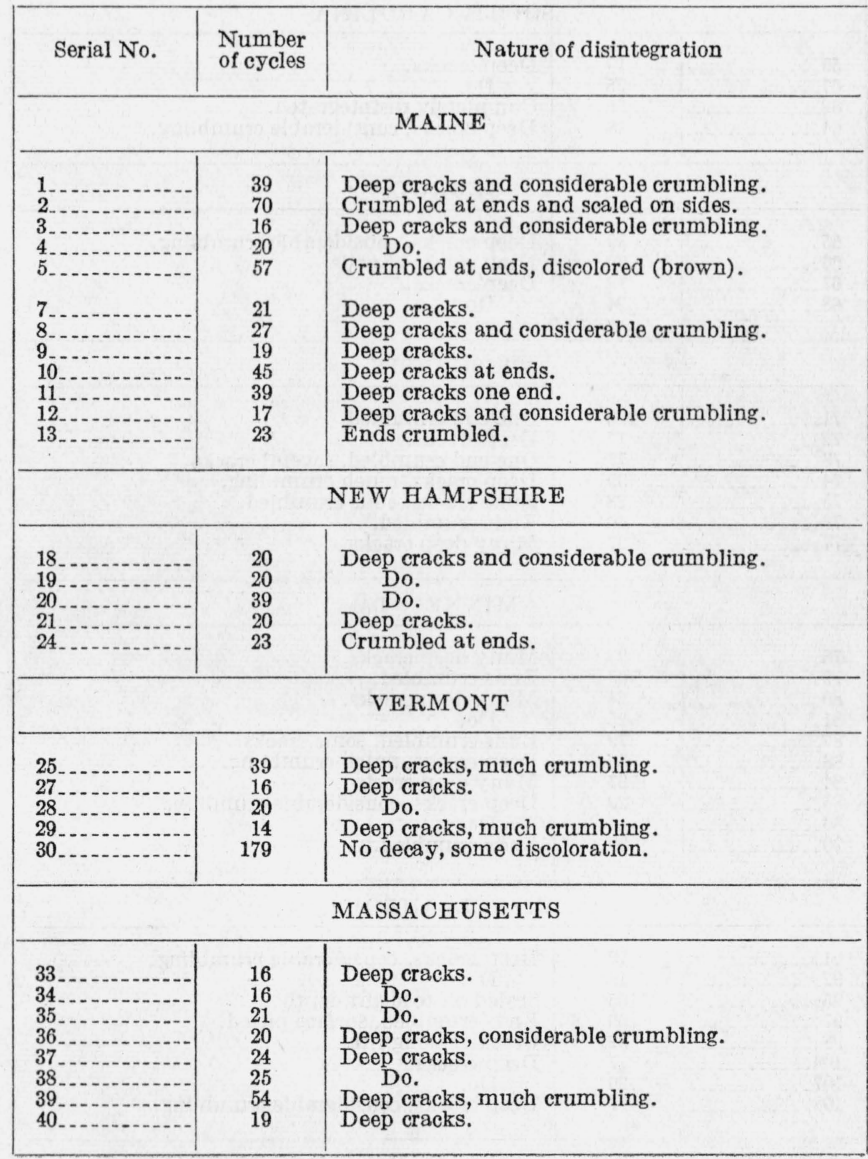


TABLE 10.-Results of crystallization test on various granites (sodium sulfate), by States-Continued

\begin{tabular}{|c|c|c|}
\hline Serial No. & $\begin{array}{l}\text { Number } \\
\text { of cycles }\end{array}$ & Nature of disintegration \\
\hline \multicolumn{3}{|r|}{ CONNECTICUT } \\
\hline 434 & $\begin{array}{l}27 \\
25 \\
45 \\
29 \\
26 \\
18\end{array}$ & $\begin{array}{l}\text { Deep cracks along rift. } \\
\text { Deep cracks. } \\
\text { Surface scaled to slight depth. } \\
\text { Deep cracks, much crumbling. } \\
\text { Deep cracks, ends crumbled. } \\
\text { Deep cracks, considerable crumbling. }\end{array}$ \\
\hline \multicolumn{3}{|r|}{ NORTH CAROLINA } \\
\hline $\begin{array}{l}49 \\
50 \\
52 \\
53 \\
54 \\
56\end{array}$ & $\begin{array}{r}179 \\
179 \\
17 \\
14 \\
19 \\
16 \\
97 \\
155 \\
26 \\
33\end{array}$ & $\begin{array}{l}\text { No appreciable decay. } \\
\text { One end cracked, slight decay. } \\
\text { Deep cracks. } \\
\text { Deep cracks, considerable crumbling. } \\
\text { Deep cracks. } \\
\text { Do. } \\
\text { Deep cracks, considerable crumbling. } \\
\text { Do. } \\
\text { Do. } \\
\text { Do. }\end{array}$ \\
\hline \multicolumn{3}{|r|}{ SOUTH CAROLINA } \\
\hline 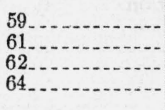 & $\begin{array}{l}19 \\
25 \\
56 \\
18\end{array}$ & $\begin{array}{l}\text { Deep cracks. } \\
\text { Do. } \\
\text { Completely disintegrated. } \\
\text { Deep cracks, considerable crumbling. }\end{array}$ \\
\hline \multicolumn{3}{|r|}{ GEORGIA } \\
\hline $\begin{array}{l}65 \\
66 \\
68 \\
68 \\
-\end{array}$ & $\begin{array}{l}39 \\
29 \\
19 \\
24\end{array}$ & $\begin{array}{l}\text { Deep cracks, considerable crumbling. } \\
\text { Deep cracks at ends. } \\
\text { Deep cracks. } \\
\text { Do. }\end{array}$ \\
\hline \multicolumn{3}{|r|}{ WISCONSIN } \\
\hline $\begin{array}{l}71 \\
73 \\
74 \\
76 \\
76 \\
77\end{array}$ & $\begin{array}{r}296 \\
17 \\
77 \\
39 \\
23 \\
66 \\
27\end{array}$ & $\begin{array}{l}\text { One end crumbled. } \\
\text { Deep cracks. } \\
\text { One end crumbled, several cracks. } \\
\text { Deep cracks, much crumbling. } \\
\text { Some cracks, ends crumbled. } \\
\text { Ends crumbled. } \\
\text { Many deep cracks. }\end{array}$ \\
\hline \multicolumn{3}{|r|}{ MINNESOTA } \\
\hline $\begin{array}{l}78 \ldots \\
80 \\
81 \\
82 \\
83 \\
84 \\
85 \\
86 \\
90 \\
90\end{array}$ & $\begin{array}{l}25 \\
66 \\
24 \\
25 \\
79 \\
39 \\
27 \\
20 \\
18 \\
39\end{array}$ & $\begin{array}{l}\text { Many deep cracks. } \\
\text { Ends crumbled. } \\
\text { Many deep cracks. } \\
\text { Do. } \\
\text { Ends crumbled, some cracks. } \\
\text { Deep cracks, much crumbling. } \\
\text { Many deep cracks. } \\
\text { Deep cracks, considerable crumbling. } \\
\text { Do. } \\
\text { Ends crumbled. }\end{array}$ \\
\hline \multicolumn{3}{|r|}{ MISCELLANEOUS } \\
\hline $\begin{array}{l}91 . \\
92 \\
95 \\
97 \\
98 \\
106 \\
107 \\
108 \\
\end{array}$ & $\begin{array}{r}18 \\
18 \\
155 \\
56 \\
32 \\
17 \\
20 \\
17\end{array}$ & $\begin{array}{l}\text { Deep cracks, considerable crumbling. } \\
\text { Do. } \\
\text { Scaled off to slight depth. } \\
\text { Ends crumbled, surface pitted. } \\
\text { Cracked along rift. } \\
\text { Deep cracks. } \\
\text { Do. } \\
\text { Deep cracks, considerable crumbling. }\end{array}$ \\
\hline
\end{tabular}


(c) TEMPERATURE AND MOISTURE CYCLE TESTS

Forty-seven samples were subjected to a test in which the specimens were soaked in water for $17 \mathrm{hr}$, then dried at $105^{\circ} \mathrm{C}$ for $7 \mathrm{hr}$. This cycle was repeated 1,000 times, the object being to determine if any mineral alterations occur within the stone which bring about disintegration. The changes were studied by means of weight, absorption, and water-transmission tests. The results are given in table 11. Most samples showed slight increases in weight, considerable increases in absorption, and large increases in water-transmission rates. The original water-transmission rate was not determined on the same specimen that was subjected to soaking and drying, but on another core from the same block of stone.

TABLE 11.-Changes in absorption, weight and water transmission of various granites, by States, after 1,000 cycles of soaking and drying

\begin{tabular}{|c|c|c|c|c|c|c|c|}
\hline \multirow{2}{*}{ Serial No. } & \multicolumn{3}{|c|}{ Absorption } & \multirow{2}{*}{$\begin{array}{c}\text { Change } \\
\text { in weight }\end{array}$} & \multicolumn{3}{|c|}{ Water transmission } \\
\hline & Original & Final & Change & & Original & Final & Change \\
\hline \multicolumn{8}{|c|}{ MAINE } \\
\hline $\begin{array}{l}1 \\
1 \\
3 \\
9^{3} \\
10 \\
11 \\
16\end{array}$ & $\begin{array}{r}\% \\
0.23 \\
.26 \\
.21 \\
.23 \\
.23 \\
.22 \\
.31 \\
.32 \\
.32\end{array}$ & $\begin{array}{r}\% \\
0.29 \\
.30 \\
.23 \\
.26 \\
.26 \\
.26 \\
.37 \\
.37 \\
.42\end{array}$ & $\begin{array}{l}\% \\
+26 \\
+15 \\
+10 \\
+13 \\
+13 \\
+18 \\
+19 \\
+16 \\
+31\end{array}$ & $\begin{array}{r}\% \\
+0.05 \\
+.03 \\
+.04 \\
+.04 \\
+.02 \\
+.06 \\
+.04 \\
+.06 \\
+.04\end{array}$ & $\begin{array}{l}\text { g. } \\
1.19 \\
3.57 \\
1.04 \\
1.28 \\
0.57 \\
.38 \\
1.15 \\
8.84 \\
1.89\end{array}$ & $\begin{array}{r}g \\
1.72 \\
26.93 \\
5.34 \\
11.39 \\
17.79 \\
1.89 \\
21.29 \\
21.58 \\
22.89\end{array}$ & $\begin{array}{r}\% \\
+45 \\
+650 \\
+410 \\
+790 \\
+3000 \\
+400 \\
+1750 \\
+144 \\
+1100\end{array}$ \\
\hline
\end{tabular}

NEW HAMPSHIRE

\begin{tabular}{r|r|r|r|r|r|r}
\hline 17 & 0.29 & 0.38 & +31 & +0.08 & 5.24 & 16.21 \\
18 & .23 & .28 & +22 & +.04 & 3.17 & 16.85 \\
23.20 & +430 \\
+2500 \\
+1200
\end{tabular}

VERMONT

\begin{tabular}{|c|c|c|c|c|c|c|c|}
\hline 25 & $\begin{array}{r}0.20 \\
.26 \\
.27\end{array}$ & $\begin{array}{r}0.26 \\
.37 \\
.30\end{array}$ & $\begin{array}{l}+30 \\
+42 \\
+11\end{array}$ & $\begin{array}{r}+0.05 \\
+.02 \\
+.01\end{array}$ & $\begin{array}{l}0.21 \\
3.45 \\
0.29\end{array}$ & $\begin{array}{r}1.10 \\
14.48 \\
.80\end{array}$ & $\begin{array}{l}+424 \\
+320 \\
+176\end{array}$ \\
\hline
\end{tabular}

MASSACHUSETTS

\begin{tabular}{r|r|r|r|r|r|r}
\hline 33 & 0.26 & 0.32 & +19 & +0.03 & 1.15 & 15.17 \\
34 & .25 & .36 & +24 & +.01 & 0.98 & 14.05 \\
+1330 \\
35 & .21 & .28 & +33 & .00 & +1200 \\
40 & .32 & .42 & +31 & +.06 & 3.43 & 4.28 \\
\hline
\end{tabular}

CONNECTICUT

\begin{tabular}{|c|c|c|c|c|c|c|c|}
\hline 4350 & $\begin{array}{r}0.19 \\
.22 \\
.31\end{array}$ & $\begin{array}{r}0.41 \\
.29 \\
.45\end{array}$ & $\begin{array}{r}+115 \\
+32 \\
+45\end{array}$ & $\begin{array}{r}+0.04 \\
+.05 \\
+.06\end{array}$ & $\begin{array}{r}0.48 \\
.30 \\
.38\end{array}$ & $\begin{array}{r}2.83 \\
7.27 \\
16.85\end{array}$ & $\begin{array}{r}+490 \\
+2300 \\
+4300\end{array}$ \\
\hline
\end{tabular}

NORTH CAROLINA

\begin{tabular}{|c|c|c|c|c|c|c|c|}
\hline 531 & $\begin{array}{r}0.20 \\
.25 \\
.29\end{array}$ & $\begin{array}{r}0.29 \\
.29 \\
.31\end{array}$ & $\begin{array}{r}+45 \\
+16 \\
+7\end{array}$ & $\begin{array}{r}+0.01 \\
+.03 \\
+.04\end{array}$ & $\begin{array}{r}9.16 \\
13.49 \\
1.79\end{array}$ & $\begin{array}{l}28.92 \\
18.59 \\
12.51\end{array}$ & $\begin{array}{r}+220 \\
+38 \\
+600\end{array}$ \\
\hline
\end{tabular}


TABLE 11.-Changes in absorption, weight and water transmission of various granites, by States, after 1,000 cycles of soaking and drying-Continued

\begin{tabular}{|c|c|c|c|c|c|c|c|}
\hline \multirow{2}{*}{ Serial No. } & \multicolumn{3}{|c|}{ Absorption } & \multirow{2}{*}{$\begin{array}{l}\text { Change } \\
\text { in weight }\end{array}$} & \multicolumn{3}{|c|}{ Water transmission } \\
\hline & Original & Final & Change & & Original & Final & Change \\
\hline 69 & $\begin{array}{l}\% \\
0.25 \\
.26\end{array}$ & $\begin{array}{l}\% \\
0.39 \\
.34\end{array}$ & $\begin{array}{l}\% \\
+56 \\
+31\end{array}$ & $\begin{array}{r}\% \\
+0.05 \\
+.06\end{array}$ & $\begin{array}{l}g \\
1.55 \\
1.75\end{array}$ & $\begin{array}{l}g \\
17.96 \\
22.09\end{array}$ & $\begin{array}{l}\% \\
+1060 \\
+1160\end{array}$ \\
\hline \multicolumn{8}{|c|}{ GEORGIA } \\
\hline $\begin{array}{l}65 \\
66 \\
67 \\
68\end{array}$ & $\begin{array}{r}0.30 \\
.24 \\
.19 \\
.26\end{array}$ & $\begin{array}{l}0.43 \\
.39 \\
.28 \\
.35\end{array}$ & $\begin{array}{l}+43 \\
+63 \\
+47 \\
+35\end{array}$ & $\begin{array}{l}+0.08 \\
+.07 \\
+.03 \\
+.06\end{array}$ & $\begin{array}{r}0.72 \\
.14 \\
4.17 \\
1.21\end{array}$ & $\begin{array}{l}12.54 \\
21.02 \\
27.40 \\
21.85\end{array}$ & $\begin{array}{r}+1640 \\
+14900 \\
+560 \\
+1700\end{array}$ \\
\hline
\end{tabular}

WISCONSIN

\begin{tabular}{r|r|r|r|r|r|r}
\hline 71 & 0.05 & 0.10 & +100 & -0.01 & 0.24 & 1.83 \\
+660 \\
+58 \\
76
\end{tabular}

MINNESOTA

\begin{tabular}{|c|c|c|c|c|c|c|c|}
\hline 89 & $\begin{array}{l}0.11 \\
.10 \\
.10 \\
.19 \\
.07 \\
.19\end{array}$ & $\begin{array}{l}0.17 \\
.14 \\
.15 \\
.26 \\
.11 \\
.27\end{array}$ & $\begin{array}{l}+55 \\
+40 \\
+50 \\
+37 \\
+57 \\
+42\end{array}$ & $\begin{array}{r}-0.01 \\
-.02 \\
-.04 \\
+.05 \\
-.02 \\
+.05\end{array}$ & $\begin{array}{r}0.16 \\
.42 \\
.29 \\
.70 \\
.14 \\
1.68\end{array}$ & $\begin{array}{l}5.49 \\
.48 \\
2.00 \\
2.07 \\
1.18 \\
5.26\end{array}$ & $\begin{array}{r}+3300 \\
+14 \\
+590 \\
+196 \\
+740 \\
+210\end{array}$ \\
\hline
\end{tabular}

MISCELLANEOUS

\begin{tabular}{r|r|r|r|r|r|r}
\hline 91 & 0.22 & 0.27 & +23 & +0.02 & 1.89 & 16.58 \\
3.01 & +780 \\
106 & .14 & .18 & +29 & -.07 & 0.36 & 8.740 \\
+250 & .15 & .21 & +40 & -.03 & 2.51 & 8.79 \\
\hline
\end{tabular}

In some specimens cracks were plainly visible. Several specimens did not show cracks; but when water passed through them, it caused several narrow bands of discoloration on the surface, indicating that small cracks had developed. Such results, along with the large increases in transmission rates for most samples, seem to show that the granites undergo changes caused by temperature and moisture cycles when the temperatures are above the freezing point. However, none of the specimens showed the scaling condition referred to as occurring on the lower courses of granite buildings. Most of the specimens increased in weight which indicates that the changes may have been caused by hydration, oxidation, or sulfation. Apparently there is no relation between the results of this test and the amount of calcite or pyrite present in the sample.

In order to determine if a temperature cycle alone would show effects like those observed in the soaking and drying test, 3 specimens were subjected to 2,000 heating and cooling cycles. The specimens were of the same type and size as those used in the soaking and drying 
tests. They were heated to $105^{\circ} \mathrm{C}$ and cooled to approximately $-10^{\circ} \mathrm{C}$. Except for a small amount of condensation on the surface when the specimens were removed from the freezing chamber, there was no moisture involved in these tests. None of the specimens showed cracks or increases in water-transmission values, and apparently there was no injury to the granite.

\section{SUMMARY}

Samples of granite were collected from 17 States for a comparative study of such characteristics as are of interest in connection with various uses of this material. Microscopic studies were made on samples of those granites for which data on the mineral constituents were not available in the literature. Tests for compressive strength and absorption were made on 116 samples and their porosity and density determined. Strength was determined on specimens in the dry and in the wet condition and also by applying the load perpendicular and parallel to the rift. Absorption tests were made for three periods of immersion, namely 2 days, 14 days, and 1 year. The flexural strength, shearing strength, modulus of elasticity, toughness, and abrasive hardness, were determined on a few selected samples. Elasticity in flexure of two samples was studied under various conditions of loading, and typical load-deflection curves for these are given. Durability studies were made by means of various laboratory tests as well as by observations on structures.

The compressive strength for individual specimens ranged from 5,900 to $60,000 \mathrm{lb} /$ in. $^{2}$ and averaged $24,500 \mathrm{lb} /$ in. ${ }^{2}$ for specimens tested dry and loaded perpendicular to the rift. The results show that, for some granites, different test pieces of the same sample vary considerably in strength, but the difference between the average of all tests on a sample and the highest or lowest individual result was usually less than 10 percent. Although some granites appear to be stronger when loaded perpendicular to than when loaded parallel to the rift, these differences were not sufficiently consistent to be significant. Compressive strengths of wet specimens averaged about 12 percent lower than those of dry specimens.

The porosity values ranged from 0.40 to 3.84 percent and averaged 1.29 percent. Absorption tests show that the specimens did not become saturated with water even after 1 year of immersion, and for a larger portion of the specimens the pores were less than half filled at the end of this time.

A test was devised for comparing the rates at which moisture rises through the pores of various granites by capillarity. The results are believed to give information on pore structure that is not indicated by absorption or porosity tests. This test was used as a measure of deterioration in some of the weathering tests, and it may be useful as a means of checking the uniformity of granite shipments.

Extensive freezing tests have indicated that granite is not seriously affected by frost action. Samples of weathered granite from three buildings were studied to determine the causes of scaling. These studies indicated that the formation of calcium sulfate, from the reaction of acids of sulfur in the air on calcareous minerals in the granite, is the cause of scaling. 
Important structures, showing the use of various granites, have been listed as an aid in future studies of the performance of these materials in service.

The authors express their appreciation to the granite producers for cooperation in supplying samples, to J. J. Tregoning for chemical studies and assistance in the petrographic work, and to the United States Bureau of Public Roads for abrasion tests on certain materials with the Dorry apparatus.

Washington, May 31, 1940. 